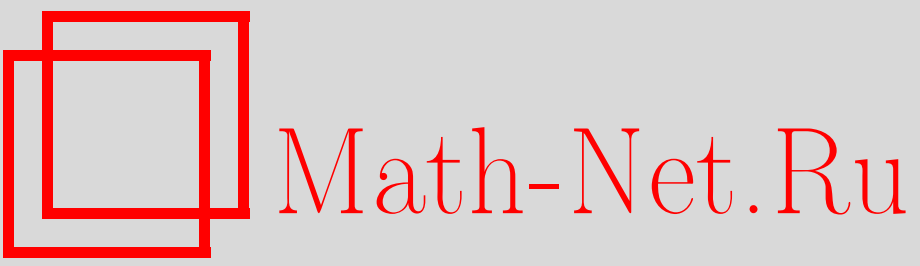

Л. Д. Беклемишев, Схемы рефлексии и алгебры доказуемости в формальной арифметике, УМH, 2005, том 60, выпуск 2, 3-78

DOI: https://doi.org/10.4213/rm1401

Использование Общероссийского математического портала Math-Net.Ru подразумевает, что вы прочитали и согласны с пользовательским соглашением

http://www.mathnet.ru/rus/agreement

Параметры загрузки:

IP: 3.85 .73 .92

26 апреля 2023 г., 12:57:40 


\title{
СХЕМЫ РЕФЛЕКСИИ И АЛГЕБРЫ ДОКАЗУЕМОСТИ В ФОРМАЛЬНОЙ АРИФМЕТИКЕ
}

\author{
Л. Д. БЕКЛЕМИШЕВ
}

Настоящая работа посвящена исследованию схем рефлексии для фрагментов формалшной арифметики Пеано и применению этих схем к вопросам сравнения и классификации арифметических теорий.

Библиография: 95 названий.

\section{СОДЕРЖАНИЕ}

Введение

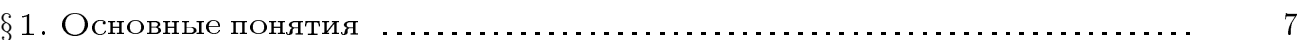

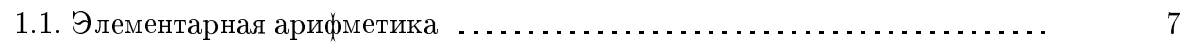

1.2. Формула доказуемости и теоремы Гёделя ....................... 9

1.3. Логика доказуемости ... . . . . . .

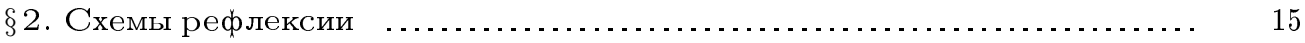

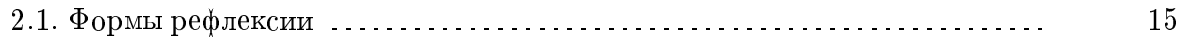

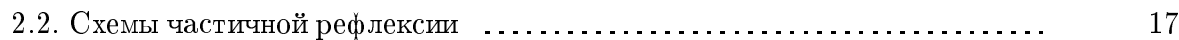

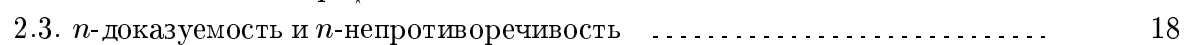

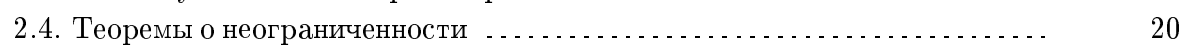

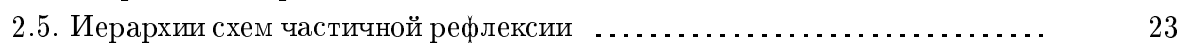

§3. Доказуемо тотальные вычислимые функции и фрагменты РА . ....... 30

3.1. Фрагменты РА .............................................. 30

3.2. Доказуемо тоталшные вьчислимые функции и 1-непротиворечивость .. 32

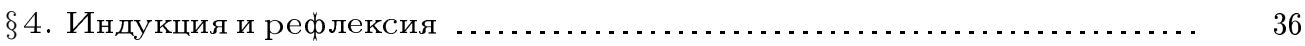

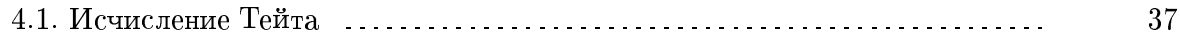

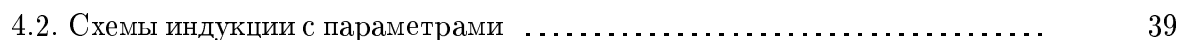

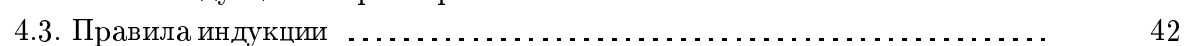

4.4. Беспараметрическая индукция …............................ 46

4.5. Схемы и правила рефрлексии . ................................ 50

$\S 5$. Алгебры доказуемости и ординальный анализ РА $\ldots \ldots \ldots \ldots \ldots \ldots \ldots \ldots . . \ldots 5$

5.1. Алгебры Линденбаума ...................................... 53

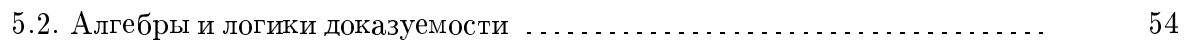

5.3. Градуированные алгебры доказуемости и логика Джапаридзе ....... 55

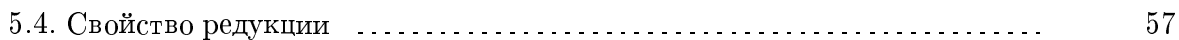


5.5. Замкнутые формулы GLP и система ординальных обозначений для ор-

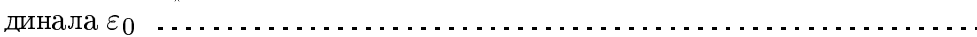

5.6. Доказательство непротиворечивости РА в стиле Генцена ... . . . . . . . . .

5.7. Невьводимое в РА комбинаторное утверждение . . . . . . . . . . . . . . . . . .

5.8. Независимость WP

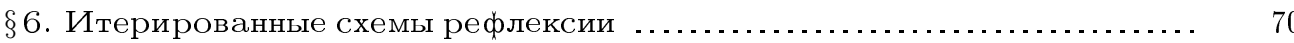

6.1. Гладкие прогрессии Тьюринга ............................... 71

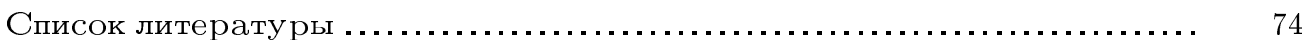

\section{Введение}

Как известно, теоремы Гёделя о неполноте показывают, что нет какой-либо одной привилегированной универсальной аксиоматической системы: все перечислимые непротиворечивые системы неполны. Формальные теории различаются как по выразительньм средствам языка, так и по логической силе заложенных в их аксиомах принципов. Кроме того, даже для теорий с одним и тем же объемом выразимых и доказуемых утверждений свойства выводов, в частности их сложность, могут быть существенно различными. Отсюда естественно возникают вопросы о критериях выбора какой-либо аксиоматической системы и о сравнении и классификации различных систем.

В теории доказательств накоплен значительньй опыт исследовани конкретных формальных теорий таких, как арифметика Пеано или определенные фрагменты арифметики второго порядка и теории множеств (см., например, обзор [68]). Эти исследования, в частности, говорят о том, что "сила" достаточно богатой в выразительном смысле теории характеризуется некоторыми счетными ординалами. Для конкретных теорий в ряде случаев эти ординалы могут быть явно вычислены, что составляет предмет изучения так назьваемого ординального анализа аксиоматических систем [67], [72], [73].

Несмотря на обилие накопленного материала и глубину полученных результатов в этой области имеется ряд проблем принципиального характера.

Во-первых, нет ясного понимания, в каких терминах должны описываться ординалы формальных теорий. В частности, нет обшего ответа на вопрос, что означает "вычислить ординал теории". Эта проблема известна с 50-х годов XX века в различных вариантах как проблема естественности ординальных обозначений [48], [49], [33]. $\mathrm{K}$ настояшему времени известны лишь подходящие естественные описания ординалов для ряда важных конкретных теорий. Это, к сожалению, еще не позволяет говорить об обшности классификащии теорий по их ординалам.

Во-вторых, ординалы даже для конкретных, но сильных теорий таких, как арифометика второго порядка (с полной схемой свертьвания) или теория множеств Цермело-Френкеля, до сих пор не вычислены. Эти вопросы также являются одними из наиболее известных и трудных открытых проблем в математической логике.

Технические трудности, связанные с программой ординального анализа, усилили в последнее время интерес специалистов к менее традиционным подходам к этим вопросам и к попыткам упростить и лучше понять применяемые методы. Среди таких

Работа выполнена при поддержке Российского фонда фундаменталшных исследований и Совета по грантам ведущих научных школ. 
попыток можно отметить работы [26], [27], где устанавливается связь между процедурами устранения сечения по Генщену и по Новикову-Шютте; работы [9], [10], [8] по теоретико-модельному подходу к ординальному анализу; работы [58], [2], [3], [91] по так назьваемому методу $\varepsilon$-подстановок.

В работах автора [20], [21] был предложен новьй подход к ординальному анализу формальной арифметики Пеано, основанный на схемах рефлексии и аппарате так называемых алгебр доказуемости. В настоящей работе мы систематизируем имеюшиеся результаты в этом направлении, а также даем обзор результатов, касающихся применения схем рефлексии к изучению фрагментов арифметики.

Схемы рефлексии возникли в математической логике вскоре после доказательства Гёделем его фундаментальных теорем о неполноте [36]. ${ }^{1}$ Для данной теории $T$ эти схемы представляют собой варианты формализации утверждения "если формула $\varphi$ доказуема в $T$, то ч истинна". Они дают примеры истинных, но недоказуемых утверждений, обобщающих первьй известньй пример такого рода - гёделевскую формулу непротиворечивости теории $T$.

А. Тьюринг [92] ввел в рассмотрение прогрессии теорий, получаемые итерированием процесса пополнения теории схемой рефлексии, и предложил возможньй подход к ординальной классификации арифметических теорий на основе таких прогрессий. В дальнейшем этот подход был проанализирован и развит С. Феферманом в [32]. Однако построенные Тьюрингом и Феферманом примеры показали, что на пути подобной классификации встают сушественные трудности, связанные, в частности, с упомянутым выше вопросом о естественном представлении ординалов в арифметике.

Г. Крайзель и А. Леви в [50] показали, что схемы рефлексии являются удобным инструментом для изучения вопросов логической сложности аксиоматизации формальных теорий. Ими была доказана дедуктивная эквивалентность так называемой равномерной схемы рефлексии для примитивно рекурсивной арифметики и полной схемы индукции, откуда, в частности, вытекает невозможность задания арифметики Пеано множеством аксиом ограниченной кванторной сложности. В этой же работе была доказана эквивалентность схемы трансфинитной индукции до ординала $\varepsilon_{0}$ и равномерной схемы рефлексии для арифметики Пеано. В дальнейшем были установлены тесные связи между схемами рефлексии и другими истинными невьводимьми утверждениями, включая известный комбинаторный принщип Париса-Харрингтона. Обзор ранних (до 1977 г.) результатов, касаюшихся схем рефлексии, можно найти в работе К. Сморинского [84].

В настоящей работе демонстрируется, что систематическое использование схем рефлексии позволяет получить самые разнообразные результаты о строении данной теории и о ее соотношении с другими теориями. В частности, мы получаем этим способом основные известные результаты о независимости, аксиоматизируемости, консервативности и классах доказуемо тотальных вычислимых функций для фрагментов РА, задаваемых различными ограничениями схемы индукции над элементарной арифметикой.

\footnotetext{
${ }^{1}$ Схемы реффлексии появились впервые, по-видимому, в работе Дж.Б. Россера 1937 г. [76]. При этом Россер ссылается на неопубликованные результаты Клини, рассмотревшего в 1935 г. вариант логического правила, эквивалентный, в современной терминологии, равномерной схеме реф̆лексии (см. ниже п. 2.1).
} 
Алгебры доказуемости. Конечную схему рефлексии $R$ над теорией $T$ можно рассматривать как оператор, действуюший на булевой алгебре Линденбаума теории $T$. Рассмотрение таких операторов естественным образом приводит к алгебраическим структурам, обогашающим алгебру Линденбаума, которые мы называем алгебрами доказуемости [21]. Простейшим примером такой структуры является алгебра Магари теории $T$, где в качестве $R$ рассматривается гёделевская формула непротиворечивости (или двойственная ей формула доказуемости) в $T$ [55]. Многообразие, порожденное такими алгебрами, активно изучалось в контексте так называемой логики доказуемости (см. [87], [25], а также [80], [82]). Используемое в настоящей работе обобщение логики доказуемости, соответствуюшее более сильньм схемам рефлексии, было исследовано в [43], [41].

В [21] было показано, что алгебры доказуемости тесно связаны с ординалами аксиоматических систем, играющими важную роль в теории доказательств. Обнаружившиеся интересные связи позволяют по новому взглянуть на ряд классических результатов в этой области. В частности, из алгебры доказуемости для элементарной арифметики можно каноническим образом извлечь стандартную систему обозначений для ординала $\varepsilon_{0}$ и на этой основе получить новое доказательство непротиворечивости арифметики Пеано с помошью трансфинитной индукции в стиле Генцена. Изучение алгебр доказуемости позволяет также дать характеризацию класса доказуемо тотальных вычислимых функций арифметики Пеано и приводит к новым простьм примерам утверждений комбинаторного характера, не выводимьм в арифметике Пеано [20].

Содержание работы. В $\S 1$ вводятся основные понятия, касаюшиеся формальной арифметики, рассматриваются свойства формулы доказуемости, а также приводятся короткие доказательства теорем Гёделя, Россера и Лёба.

В 22 изучаются обшие свойства схем локальной и равномерной рефлексии и доказываются так назьваемые теоремы Крайзеля-Леви о неограниченности.

В 33 приводятся простейшие результаты о доказуемо тотальных вычислимых функциях теорий и их связи со схемами рефилексии.

В $₫ 4$ устанавливаются результаты о связи схем рефлексии и индукции в арифметике. Показано, что естественные фрагменты РА, задаваемые различными ограничениями схемы индукции, в точности соответствуют (дедуктивно эквивалентны) определенным схемам реффексии над элементарной арифметикой ЕА. Здесь также получен важньй для дальнейшего результат о консервативности схем над правилами рефлексии - так назьваемое свойство редукции.

Вместе с результатами $\S 2$ это позволяет единым методом получить ответ на ряд вопросов о свойствах фрагментов РА. Так, следствие 4.20 дает характеризацию доказуемо тотальных вычислимых функций в теории, аксиоматизируемой схемой индукции для $\Pi_{2}$-формул без параметров. Класс таких функций совпадает с примитивно рекурсивными функциями. Расширение этой теории схемой индукции для $\Sigma_{1}$-формул с параметрами имеет более широкий класс доказуемо тотальных вычислимых функций, совпадаюший с классом дважды рекурсивных функций в смысле Р. Петер (следствие 6.3). Эти результаты, полученные в [18], по-видимому, являются наиболее интересными приложениями техники $\S \S 2$ и 4.

В $\S 5$ исследуется понятие алгебры доказуемости и на этой основе излагается подход к ординальному анализу арифметики Пеано. В частности, мы даем новое доказатель- 
ство непротиворечивости РА с помошњю трансфинитной индукции до ординала $\varepsilon_{0}$. Также мы приводим простой пример истинного утверждения комбинаторного характера, недоказуемого в арифметике Пеано и естественно возникающего из алгебр доказуемости. Наконец, мы устанавливаем связь между алгебрами доказуемости и трансфинитньми прогрессиями итерированных схем рефлексии по Тьюрингу-Феферману. Отсюда мы получаем характеризацию $\Pi_{n}$-следствий РА в терминах таких прогрессий.

\section{$\S 1$. Основные понятия}

1.1. Элементарная арифметика. Язьк элементарной арифметики - это язык первого порядка, содержаший бинарные предикатные символы $=$ и $\leqslant$, бинарные функциональные символы + и · унарные функциональные символы $S$ и ехр и константу 0. Стандартная модель арифметики - это модель с универсумом $\mathbb{N}=\{0,1,2, \ldots\}$, на котором все символы интерпретируются естественным образом, в частности, $S$ есть функция следовани $S(x)=x+1$ и ехр есть показательная функция $\exp (x)=2^{x}$.

Формулы этого языка называются арифметическими. Мы будем следовать обычным соглашениям об опускании излишних скобок в формулах и пользоваться обшепринятыми сокрашениями, т.е. будем писать, например, $x<y$ вместо $x \leqslant y \wedge \neg x=y$, $2^{x}$ вместо $\exp (x)$ и т. д.

Выражения $\forall x \leqslant t A(x)$ и $\exists x \leqslant t A(x)$ являются сокрашениями для формул $\forall x$ $(x \leqslant t \rightarrow A(x))$ и $\exists x \quad(x \leqslant t \wedge A(x))$ соответственно (где $t-$ любой терм, не содержаший переменной $x$ ). Вхождения кванторов такого вида называются ограниченными ; ограниченными или элементарнылми формулами назьваются арифметические формулы, все вхождения кванторов в которые ограничены. Заметим, что по определению бескванторные формулы являются элементарными. Аналогично определяются выражения $\forall x<t A(x)$ и $\exists x<t A(x)$. Элементарные предикаты или отношения это отношения, определимые элементарньми формулами в стандартной модели арифметики.

Произвольные арифметические формулы классифицируются по своей логической сложности в классическую арифметическую иерархию. Для $n \geqslant 0$ классы $\Sigma_{n^{-}}$ и $\Pi_{n}$-формул определяются индуктивно следующим образом. $\Sigma_{0^{-}}$и $\Pi_{0}$ форормулы - это элементарные формулы. $\Sigma_{n+1}$-формулы - это формулы вида $\exists x_{1} \ldots \exists x_{m}$ $A\left(x_{1}, \ldots, x_{m}\right)$, где $A$ - некоторая $\Pi_{n}$-формула. $\Pi_{n+1}$-формулы - это формулы вида $\forall x_{1} \ldots \forall x_{m} A\left(x_{1}, \ldots, x_{m}\right)$, где $A$ - некоторая $\Sigma_{n}$-формула.

По теореме о предваренной нормальной форме, всякая арифметическа формула логически эквивалентна $\Sigma_{n}$-формуле для некоторого $n$. Допуская вольность речи, мы будем иногда назьвать $\Sigma_{n}$-формулами также и формулы, логически эквивалентные $\Sigma_{n}$-формулам в смысле нашего официального определения.

С вычислительной точки зрения наиболее важным классом формул является $\Sigma_{1}$. Предикат является $\Sigma_{1}$-определимым в стандартной модели ариффметики, если и только если он (рекурсивно) перечислим. Элементарные предикаты разрешимы, причем грубая оценка сложности разрешающей процедуры дает верхнюю оценку порядка $2_{n}^{x}$ на число ее шагов, где $n$ - некоторая константа, а $x$ - размер входа. Здесь $2_{n}^{x}$ означает функцию

$$
2_{0}^{x}=x ; \quad 2_{n+1}^{x}=2^{2_{n}^{x}}
$$


Функции вида $2_{n}^{x}$ для фиксированного $n$ назьваются мультиэкспоненииальнылми; таким образом, всякий элементарньй предикат разрешим за мультиэкспоненциальное время. Обратное также верно: всякий предикат, разрешимый за мультиэкспоненциальное время, элементарен (см. [29]).

Арифметика Пеано РА - теория первого порядка с равенством, сформулированная в арифметическом языке и задаваемая, помимо логических аксиом и аксиом равенства, следуюшими математическими аксиомами:

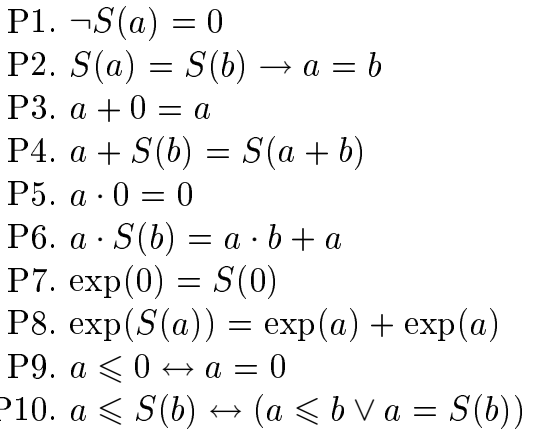

вместе со следующей схемой аксиом индукиии, для всех арифметических формул $\varphi(x)$ (возможно, содержащих параметры, т.е. свободные переменные помимо $x$ ):

IA: $\varphi(0) \wedge \forall x(\varphi(x) \rightarrow \varphi(S(x))) \rightarrow \forall x \varphi(x)$.

Элементарная арифметика ЕА аксиоматизируется над Р1-P10 схемой индукции для элементарных формул $\varphi(x)$.

Элементарная арифметика представляет собой достаточно слабый фрагмент РА. Хорошо известно, что, аналогично РА, ЕА имеет эквивалентную формулировку в языке арифметики без символа экспоненты. Теория $I \Delta_{0}$ задается в этом языке аксиомами P1-P6, P9, P10 вместе со схемой индукции для $\Delta_{0}$-формул (т.е. ограниченных формул языка без ехр). График функции $2^{x}$ можно естественным образом определить некоторой $\Delta_{0}$-формулой $\operatorname{Exp}(x, y)[39 ;$ с. 299$]$. Для этой формулы в $I \Delta_{0}$ выводимы аналоги аксиом Р 7, Р8 и свойства функциональности и монотонности. Формула $\operatorname{Exp}(x, y)$ определяет интерпретацию ЕА в теории $I \Delta_{0}+\operatorname{Exp}$, где Ехр есть аксиома $\forall x \exists y \operatorname{Exp}(x, y)$, выражаюшая тотальность экспоненты [39; с. 37]. Фактически, ЕА дедуктивно эквивалентна консервативному дефинициальному расширению $I \Delta_{0}+$ Exp с помошью символа для функции ехр. Нетрудно убедиться в том, что эта интерпретация сохраняет классы $\Sigma_{n}$ и $\Pi_{n}$-форомул при $n \geqslant 1$ по модулю вьводимости в $I \Delta_{0}+$ Exp. (Класс элементарных отношений, однако, строго шире, чем класс $\Delta_{0}$-отношений. Элементарные отношени совпадают с доказуемо $\Delta_{1}$-отношениями для теории $I \Delta_{0}+$ Exp.)

В отличие от $\mathrm{PA}, I \Delta_{0}+$ Ехр и ЕА являются конечно аксиоматизируемыми теориями $[39 ;$ с. 366$])$. Нетрудно убедиться, что ЕА аксиоматизируема формулами сложности П 1 в арифметической иерархии, а $I \Delta_{0}+$ Ехр имеет сложность аксиоматизации $\Pi_{2}$ (в язьке без ехр).

Элементарными (по Кальмару) функциями назьваются функции, которые могут быть получены из функций $Z(x) \equiv 0, S,+, \cdot, 2^{x}$, функции

$$
x \doteq y:= \begin{cases}0, & \text { если } x \leqslant y, \\ x-y & \text { иначе }\end{cases}
$$


и проектируюших функщий $I_{i}^{n}\left(x_{1}, \ldots, x_{n}\right)=x_{i}$ с помошюю операций композиции и ограниченной минимизации [74]:

$$
\mu i \leqslant z R(i, \vec{x}):= \begin{cases}y, & \text { если } y \leqslant z \text { и } R(y, \vec{x}) \wedge \forall i<y \neg R(i, \vec{x}), \\ 0, & \text { если } \forall i \leqslant z \neg R(i, \vec{x}) .\end{cases}
$$

(Здесь $R$ - предикат вида $g(i, \vec{x})=0$ для некоторой ранее определенной функции $g$. .)

Класс элементарных функций обозначается $\mathscr{E}$ и совпадает с классом функций, вычислимых по Тьюрингу за мультиэкспоненциальное время (см. [29]). Класс $\mathscr{E}$ замкнут относительно операций ограниченного суммирования, ограниченного умножения и ограниченной примитивной рекурсии. Фактически, по модулю композищии и достаточно большого набора начальных функций каждая из этих операций эквивалентна ограниченной минимизации (см. [74; гл. 5]).

Символы для всех элементарных функций могут быть естественньм образом введены в консервативном дефинищиальном расширении ЕА. В этом расширении любая ограниченная формула расширенного языка эквивалентна бескванторной. Более того, само это расширение можно аксиоматизировать множеством чисто универсальных (или бескванторных) формул. С другой стороны, всякая ограниченная формула расширенного языка эквивалентна ограниченной арифметической формуле и то же самое относится к остальным классам арифметической иерархии. В частности, в дефинициальном расширении ЕА допустима индукция по ограниченным формулам расширенного языка. Таким образом, формулировки ЕА в стандартном и расширенном языках являются в сильном смысле эквивалентными, и мы часто будем их отождествлять. Вьшеприведенные утверждения являются фольклорньми; бескванторная формулировка ЕА подробно рассмотрена, например, в работе [70].

1.2. Формула доказуемости и теоремы Гёделя. Стандартная арифметизащия синтаксиса теорий первого порядка - подробно развитая в работе Фефермана [31] для случа РА, или для случая примитивно рекурсивной арифметики в книге К. Сморинского [87] - проходит и в элементарной арифметике без сушественных изменений. Это связано с тем, что все необходимые для определения основных синтаксических понятий применения оператора примитивной рекурсии фактически являются мультиэкспоненциально ограниченными и тем самым допустимьми в ЕА (см. также [70]).

Формулы арифметического языка естественным образом отождествляются со словами в некотором конечном алфавите. В свою очередь, слова могут быть естественным взаимно однозначным образом отождествлены с натуральными числами, например с помошю преобразования их в двоичные слова и последуюшего двоичного декодирования. (Заметим, что последнее легко выражается в ЕА с помощью экспоненты и арифметических операций.) Таким образом мы фиксируем единое каноническое кодирование - или гёделеву нумеращию - всех выражений арифметического языка: переменных, термов, формул и т. д. Код, или гёделев номер, выражения $\tau$ обозначается $\ulcorner\tau\urcorner$. Нетрудно убедиться в том, что при таком кодировании естественные синтаксические отношения такие, как приведенные ниже, являются элементарными (более того, их простейшие свойства, верифицируемые с помошью элементарной индукции, 
доказуемы в ЕА (см. [31])):

$x \in S t \quad$ " $x$ есть гёделев номер арифметического предложения"

$x \in \Pi_{n} \quad$ " $x$ есть гёделев номер $\Pi_{n}$-формулы"

$\log A x(x) \quad$ “ $x$ есть гёделев номер логической аксиомы”

$E q A x(x) \quad$ " $x$ есть гёделев номер аксиомы равенства"

$M P(x, y, z)$ "формула $z$ следует из формул $x$ и $y$ по правилу modus ponens"

$\operatorname{Gen}(x, y)$ “формула $y$ следует из формулы $x$ по правилу обобщения"

Мы также фиксируем взаимно однозначную элементарную функцию кодирования конечных последовательностей натуральных чисел. $\left\langle x_{1}, \ldots, x_{n}\right\rangle$ означает код последовательности $x_{1}, \ldots, x_{n}$ и является элементарной функцией $x_{1}, \ldots, x_{n}$ при каждом фиксированном $n .(x)_{i}$ означает $(i+1)$-й элемент последовательности, кодируемой числом $x$, и является элементарной функцией аргументов $x$ и $i$. 〈〉 означает код пустой последовательности. Следуюшие функции и отношения также элементарны и естественно представимы в ЕА:

$\begin{array}{ll}S e q(x) & \text { "x есть код последовательности" } \\ l h(x) & \text { длина последовательности } x \\ e n d(x) & \text { последний элемент последовательности } x \\ x * y & \text { конкатенация последовательностей } x \text { и } y\end{array}$

Мы предполагаем, что кодирование последовательностей доказуемо монотонно в том смысле, что код последовательности увеличивается при ее удлинении или увеличении любого из элементов.

Теориями мы будем называть теории первого порядка с равенством. Как правило, если не оговорено противное, мы будем подразумевать, что теории сфформулированы в арифметическом языке и содержат ЕА. Теория $T$ назьвается элементарно аксиоматизированной, если множество гёделевых номеров ее (математических) аксиом элементарно, т.е. определено некоторой ограниченной формулой $\mathrm{Ax}_{T}(x)$. Все обычные теории, такие как EA, PA и другие, встречаюшиеся в данной работе, являются элементарно аксиоматизированными. Более того, в силу известной леммы Крейга [31] всякая перечислимая теория имеет элементарную аксиоматизацию, и, таким образом, элементарная аксиоматизируемость не является существенно ограничительньм условием. Теория $T$ называется корректной, если все теоремы $T$ истинны в стандартной модели арифметики. Теория $T \Sigma_{n}$-корректна, если все ее теоремы арифметической сложности $\Sigma_{n}$ истинны в стандартной модели. Теории $T$ и $U$ назьваются дедуктивно эквивалентньми, если у них одно и то же множество теорем (обозначается $T \equiv U)$. Если $T$ и $U$ - элементарно аксиоматизированные теории, то через $T+U$ обозначается теория, аксиомы которой задаются формулой $A x_{T}(x) \vee \operatorname{Ax}_{U}(x)$.

С любой элементарно аксиоматизированной теорией $T$ связывается предикат “y кодирует вывод формуль $x$ в теории $T$ ”, задаваемьй элементарной формулой $\operatorname{Prf}_{T}(y, x)$. Формула $\operatorname{Prf}_{T}(y, x)$ строится следуюшим образом, буквально следуя гильбертовскому определению вьвода в логике первого порядка:

$$
\begin{aligned}
& \operatorname{Prf}_{T}(y, x):=\operatorname{Seq}(y) \wedge \operatorname{end}(y)=x \wedge \\
& \forall n<\operatorname{lh}(y)[ \log A x\left((y)_{n}\right) \vee \operatorname{EqAx}\left((y)_{n}\right) \vee \operatorname{Ax}_{T}\left((y)_{n}\right) \\
& \vee \exists i, j<n M P\left((y)_{i},(y)_{j},(y)_{n}\right) \\
&\left.\vee \exists i<n \operatorname{Gen}\left((y)_{i},(y)_{n}\right)\right] .
\end{aligned}
$$


Другими словами, $y$ кодирует последовательность формул, заканчивающуюся на $x$, такую, что каждый элемент $y$ является или логической аксиомой, или аксиомой равенства, или математической аксиомой $T$, или следует из предыдущих формул по правилам modus ponens или обобщения.

Гёделевская формула $\square_{T}(x)$ доказуемости в $T$ определяется как $\exists y \operatorname{Prf}_{T}(y, x)$. Мы используем следующие стандартные сокрашения. Замкнутые термы вида $S(S(\ldots S(0) \ldots))$ ( $n$ раз) назьваются нумералами и обозначаются $\bar{n}$. Мы также опускаем черту в тех случаях, когда $n$ невозможно спутать с переменной. Если $\varphi-$ формула, мы пишем $\square_{T} \varphi$ вместо $\square_{T}(\overline{\ulcorner\varphi\urcorner})$. Символ $\perp$ означает логическую связку “ложь”, а $\operatorname{Con}(T)$ есть формула непротиворечивости теории $T$, определяемая как $\neg \square_{T} \perp$. Выражение $\left\ulcorner\sigma\left(\dot{x}_{1}, \ldots, \dot{x}_{k}\right)\right\urcorner$ означает естественный элементарный определимый терм для функции, сопоставляющей последовательности $n_{1}, \ldots, n_{k}$ гёделев номер $\left\ulcorner\sigma\left(\bar{n}_{1}, \ldots, \bar{n}_{k}\right)\right\urcorner$ результата подстановки нумералов $\bar{n}_{1}, \ldots, \bar{n}_{k}$ вместо переменных $x_{1}, \ldots, x_{k}$ в формулу $\sigma$. Мы также пишем $\square_{T} \sigma\left(\dot{x}_{1}, \ldots, \dot{x}_{n}\right)$ вместо $\square_{T}\left(\left\ulcorner\sigma\left(\dot{x}_{1}, \ldots, \dot{x}_{n}\right)\right\urcorner\right)$.

ПРЕДЛОЖЕНИЕ 1.1 (условия Лёба). Для любъх предложений $\varphi, \psi$

L1. $T \vdash \varphi \Longrightarrow \mathrm{EA} \vdash \square_{T} \varphi$

L2. EA $\vdash \square_{T}(\varphi \rightarrow \psi) \rightarrow\left(\square_{T} \varphi \rightarrow \square_{T} \psi\right)$

L3. EA $\vdash \square_{T} \varphi \rightarrow \square_{T} \square_{T} \varphi$

Аналогичные утверждения имеют место и для формул со свободными переменными.

$\mathrm{L} 1^{\circ} . T \vdash \varphi(x) \Longrightarrow \mathrm{EA} \vdash \square_{T} \varphi(\dot{x})$

$\mathrm{L} 2^{\circ}$. EA $\vdash \square_{T}(\varphi(\dot{x}) \rightarrow \psi(\dot{x})) \rightarrow\left(\square_{T} \varphi(\dot{x}) \rightarrow \square_{T} \psi(\dot{x})\right)$

$\mathrm{L} 3^{\circ}$. EA $\vdash \square_{T} \varphi(\dot{x}) \rightarrow \square_{T} \square_{T} \varphi(\dot{x})$

$\mathrm{L} 4^{\circ}$. EA $\vdash \square_{T} \forall x \varphi(x) \rightarrow \forall x \square_{T} \varphi(\dot{x})$

Заметим, что $\mathrm{L} 4{ }^{\circ}$ следует из $\mathrm{L} 1^{\circ}$ и $\mathrm{L} 2^{\circ}$ с помощью аксиомы $\forall x \varphi(x) \rightarrow \varphi(x)$. Свойства $\mathrm{L} 3$ и L $3^{\circ}$ следуют из более общего утверждения, известного как доказуемая $\Sigma_{1}-n о л-$ нота [39], [87].

ПРЕДЛОЖЕНИЕ 1.2 .

(i) Для любого $\Sigma_{1}$-предложения $\sigma$

$$
\mathrm{EA} \vdash \sigma \rightarrow \square_{T} \sigma .
$$

(ii) Для любой $\Sigma_{1}$-формуль $\sigma\left(x_{1}, \ldots, x_{n}\right)$, где все свободные переменные $\sigma$ указаны явно,

$$
\mathrm{EA} \vdash \sigma\left(x_{1}, \ldots, x_{n}\right) \rightarrow \square_{T} \sigma\left(\dot{x}_{1}, \ldots, \dot{x}_{n}\right) .
$$

Возможность формализации $\Sigma_{1}$-полноты теории $T$ в ЕA следует из наличия мультиэкспоненциальной оценки на размер ЕА-доказательства одной из формул $\varphi(\bar{n})$ или $\neg \varphi(\bar{n})$ в зависимости от $n$ для любой элементарной формулы $\varphi(x)$ (см. [87] и $[39 ;$ с. 175$])$.

Для доказательства теорем Гёделя о неполноте и многих других результатов ключевую роль играет следуюшая арифметическая лемма о неподвижной точке $[31],[87]$. 
ПРЕДЛОЖЕНИЕ 1.3.

(i) Для любой формуль $\varphi(x)$ найдется формула $\psi$, зависящая от свободных переменны $\varphi$ кроме $x$, такая, что

$$
\mathrm{EA} \vdash \psi \leftrightarrow \varphi(\ulcorner\psi\urcorner)
$$

(ii) Для любой формуль $\varphi\left(x, x_{1}, \ldots, x_{n}\right)$ найдется формула $\psi\left(x_{1}, \ldots, x_{n}\right)$ такая, что

$$
\mathrm{EA} \vdash \psi\left(x_{1}, \ldots, x_{n}\right) \leftrightarrow \varphi\left(\left\ulcorner\psi\left(\dot{x}_{1}, \ldots, \dot{x}_{n}\right)\right\urcorner\right) .
$$

Следуюшее обобщение теоремы Гёделя известно как теорема Лёба [54]. Ввиду его важности для настоящей работы мы приводим короткое доказательство.

Теорема 1 (Лёб). Для любой әлементарно аксиоматизированной теории $T$, содержащей ЕА, и любого предложения $\varphi$

$$
T \vdash \square_{T} \varphi \rightarrow \varphi \Longleftrightarrow T \vdash \varphi .
$$

ДокАЗАТЕльство. Импликация $(\Leftarrow)$ очевидна. Для доказательства противоположной импликации, с помошю леммы о неподвижной точке мы получаем предложение $\psi$ такое, что

$$
\mathrm{EA} \vdash \psi \leftrightarrow\left(\square_{T} \psi \rightarrow \varphi\right)
$$

Опираясь на условия Лёба и тот факт, что теория $T$ содержит EA, мы последовательно вьводим в $T$ :

1. $\square_{T}\left(\psi \rightarrow\left(\square_{T} \psi \rightarrow \varphi\right)\right)$

2. $\square_{T} \psi \rightarrow \square_{T}\left(\square_{T} \psi \rightarrow \varphi\right)$

3. $\square_{T} \psi \rightarrow\left(\square_{T} \square_{T} \psi \rightarrow \square_{T} \varphi\right)$

4. $\square_{T} \psi \rightarrow \square_{T} \varphi$

5. $\square_{T} \psi \rightarrow \varphi$, поскольку $T \vdash \square_{T} \varphi \rightarrow \varphi$

6. $\psi$

7. $\square_{T} \psi$

8. $\varphi$.

Подставляя $\perp$ вместо $\varphi$, как следствие получаем вторую теорему Гёделя о неполноте [36].

ТЕОРЕМА 2 (Гёдель). Для любой әлементарно аксиоматизированной теории $T$, содержащей ЕА,

(i) если $T$ непротиворечива, то $T \nvdash \operatorname{Con}(T)$;

(ii) если, кроме того, $T \quad \Sigma_{1}$-корректна, то $T \nvdash \neg \operatorname{Con}(T)$. 
ЗАмечАниЕ 1.4. Теорема Лёба может в свою очередь рассматриваться как следствие второй теоремы Гёделя для теории $T+\neg \varphi$ при условии, что аксиомы этой теории определены формулой $\mathrm{Ax}_{T+\neg \varphi}(x):=\mathrm{Ax}_{T}(x) \vee x=\ulcorner\neg \varphi\urcorner$. Для соответствующего предиката доказуемости имеем

$$
\mathrm{EA} \vdash \operatorname{Con}(T+\neg \varphi) \leftrightarrow \neg \square_{T} \varphi
$$

в силу формализуемости теоремы о дедукции в ЕА (см. [31]).

Следуюшее утверждение представляет собой формализацию теоремы Лёба и может быть выведено с помощью условий Лёба из неформализованного варианта этой теоремы (см. [25]).

СлЕДСТВИЕ 1.5. Для любого предложения $\varphi$

$$
\mathrm{EA} \vdash \square_{T}\left(\square_{T} \varphi \rightarrow \varphi\right) \rightarrow \square_{T} \varphi
$$

ДокАЗАТЕЛЬСТво. Обозначим формулу $\square_{T}\left(\square_{T} \varphi \rightarrow \varphi\right) \rightarrow \square_{T} \varphi$ через $\psi$. Опираясь на условия Лёба, нетрудно вьвести, что

$$
\mathrm{EA} \vdash \square_{T} \psi \rightarrow \psi
$$

Отсюда, по теореме $1, T \vdash \psi$, а значит, $\mathrm{EA} \vdash \square_{T} \psi$ и $\mathrm{EA} \vdash \psi$ в силу (1.1).

Приведем также известную теорему Дж. Б. Россера, обобщающую первую теорему Гёделя о неполноте [75].

Теорема 3 (Россер). Для любой непротиворечивой перечислимой теории $T$, содержащей ЕА, найдется такое $\Sigma_{1}$-предложение $\varphi$, что $T \nvdash \varphi$ и $T \nvdash \neg \varphi$.

ДокАЗАТЕльство. Рассмотрим какую-либо элементарную аксиоматизацию теории $T$. В качестве $\varphi$ возьмем неподвижную точку,

$$
\mathrm{EA} \vdash \varphi \leftrightarrow \exists y\left(\operatorname{Prf}_{T}(y,\ulcorner\neg \varphi\urcorner) \wedge \forall z<y \quad \neg \operatorname{Prf}_{T}(z,\ulcorner\varphi\urcorner)\right) .
$$

Допустим $T \vdash \neg \varphi$, тогда если $m-$ код некоторого вьвода формулы $\neg \varphi$, получаем

$$
\mathrm{EA} \vdash \operatorname{Prf}_{T}(\bar{m},\ulcorner\neg \varphi\urcorner) \wedge \forall z<\bar{m} \neg \operatorname{Prf}_{T}(z,\ulcorner\varphi\urcorner)
$$

в силу $\Sigma_{1}$-полноты, откуда вытекает $T \vdash \varphi$ по определению $\varphi$ и противоречие в $T$. Если же $T \vdash \varphi$ и $n-$ код некоторого вывода формулы $\varphi$, получаем в силу определения $\varphi$

$$
T \vdash \exists y \leqslant \bar{n} \operatorname{Prf}_{T}(y,\ulcorner\neg \varphi\urcorner) .
$$

Поскольку теория $T$ непротиворечива, то $T \nvdash \neg \varphi$, т.е. $\Sigma_{1}$-формула

$$
\forall y \leqslant \bar{n} \neg \operatorname{Prf}_{T}(y,\ulcorner\neg \varphi\urcorner)
$$

истинна. Но тогда она вьводима в ЕА, и мы получаем противоречие в $T$. 
1.3. Логика доказуемости. Базисная логика доказуемости Гёделя-Лёба GL формулируется в языке пропозищиональной логики высказываний, обогащенном унарной связкой $\square$. Формулы этого языка называем модальны.ми. Выражения $\diamond \varphi, \square^{+} \varphi$ и $\square^{n} \varphi$ суть сокрашения для $\neg \square \neg \varphi, \varphi \wedge \square \varphi$ и $\square \square . . \square \varphi$ ( $n$ раз) соответственно. Логика GL определяется следуюшими аксиомами и правилами вывода.

\section{Аксиомы:}

А1. Тавтологии булевой логики высказьваний;

A2. $\square(\varphi \rightarrow \psi) \rightarrow(\square \varphi \rightarrow \square \psi)$;

A3. $\square \varphi \rightarrow \square \square \varphi$;

A4. $\square(\square \varphi \rightarrow \varphi) \rightarrow \square \varphi$.

Правила вывода: modus ponens и $\varphi \vdash \square \varphi$ (усиление).

Арифметической реализацией языка GL мы называем любую функцию ( · )* отображающую пропозищиональные переменные во множество арифметических предложений. Для всякой модальной формулы $\varphi(\varphi)_{T}^{*}$ означает результат подстановки вместо пропозициональных переменных $\varphi$ соответствуюших арифметических предложений и расшифровки $\square$ как формулы доказуемости $\square_{T}$ :

$$
\begin{aligned}
(\varphi \rightarrow \psi)_{T}^{*} & :=\left((\varphi)_{T}^{*} \rightarrow(\psi)_{T}^{*}\right), \\
(\neg \varphi)_{T}^{*} & :=\neg(\varphi)_{T}^{*}, \\
(\square \varphi)_{T}^{*} & :=\square_{T}(\varphi)_{T}^{*} .
\end{aligned}
$$

Нетрудно видеть, что при этой интерпретации аксиомы А2, А3 и правило усиления соответствуют трем условиям Лёба, а аксиома А4 есть формализация теоремы Лёба (см. следствие 1.5). Отсюда следует, что для любой модальной формулы $\varphi$ GL $\vdash \varphi$ влечет $\mathrm{EA} \vdash(\varphi)_{T}^{*}$ для любой арифметической реализации $(\cdot)^{*}$ переменных $\varphi$. Противоположная импликация для случая $\Sigma_{1}$-корректной теории $T$ также верна. Это утверждение составляет содержание известной теоремы Р. Соловея об арифметической полноте $\mathbf{G L}$ (см. [88], [25]).

ТЕОРема 4 (Соловей). Пусть элементарно аксиоматизированная теория $T$ $\Sigma_{1}$-корректна и содержит ЕА. Тогда

$$
\text { GL } \vdash \varphi \Longleftrightarrow T \vdash(\varphi)_{T}^{*} \text { для любой реализации }(\cdot)^{*} \text { переменных } \varphi .
$$

Удобньм инструментом исследования логики доказуемости являются модели Крипке. Моделью Крипке назьвается тройка $\mathscr{W}=(W, R, \Vdash)$, где

1. $W$ есть непустое конечное множество;

2. $R$ есть отношение иррефилексивного частичного порядка на $W$;

3 . $\Vdash$ есть отношение вьнуждения между элементами $W$ (мирами) и модальными формулами такое, что

$$
\begin{aligned}
x \Vdash \neg \varphi & \Longleftrightarrow x \nVdash \varphi, \\
x \Vdash(\varphi \rightarrow \psi) & \Longleftrightarrow(x \nVdash \varphi \text { или } x \Vdash \psi), \\
x \Vdash \square \varphi & \Longleftrightarrow \forall y \in W \quad(x R y \Rightarrow y \Vdash \varphi) .
\end{aligned}
$$

Пишем $\mathscr{W} \vDash \varphi$, если $\forall x \in W \quad \Vdash \quad$. Доказательство следуюшей теоремы, принадлежашей К. Сегербергу [79], можно также найти, например, в [87], [25]. 
Теорема 5. Для любой формуль $\varphi$

$$
\mathbf{G L} \vdash \varphi \Longleftrightarrow \mathscr{W} \vDash \varphi \text { для любой модели Крипке } \mathscr{W}
$$

Эта теорема дает полезньй критерий проверки модальных формул на выводимость в GL. Таким образом, логика GL является разрешимой, полной и финитно аппроксимируемой по Крипке. Известно также, что GL обладает интерполяционным свойством Крейга и имеет естественную секвенщиальную формулировку без правила сечения.

Изучение логики доказуемости и ее обобщений представляет собой достаточно обширную область исследований, выходящую за рамки данной статьи. Этому предмету посвящены ряд обзоров [44], [7], [53], [93] и монографий [23], [87], [25]. В настоящей работе мы будем опираться на полноту GL по Крипке. Однако мы используем лишь корректность GL относительно арифметической интерпретации, т.е. не опираемся на наиболее трудную часть теоремы Соловея.

\section{$\S$ 2. Схемы рефлексии}

Для данной теории $T$ схемы рефлексии представляют собой варианты формализации утверждения о корректности $T$. Мы вьнуждены иметь дело именно со схемами, поскольку в силу известной теоремы Тарского понятие истинности арифметической формулы является невыразимым в языке арифметики. Тем самым, утверждение "любое доказуемое предложение истинно" невозможно выразить одной арифметической формулой. Поскольку корректность $T$ относительно некоторой модели влечет ее непротиворечивость, схемы реф̆лексии для $T$ недоказуемы в самой теории $T$. В этом параграфе мы рассмотрим основные формы и обшие свойства схем рефлексии.

2.1. Формы рефлексии. Пусть $T$ - элементарно аксиоматизированная теория. Две основные формы схемы рефлексии суть:

- локальная схема рефлексии $\operatorname{Rfn}(T)$ :

$$
\square_{T} \varphi \rightarrow \varphi
$$

для всех арифметических предложений $\varphi$;

- равномерная схема рефлексии $\operatorname{RFN}(T)$ :

$$
\forall x_{1} \ldots \forall x_{n}\left(\square_{T} \varphi\left(\dot{x}_{1}, \ldots, \dot{x}_{n}\right) \rightarrow \varphi\left(x_{1}, \ldots, x_{n}\right)\right)
$$

для всех арифметических формул $\varphi\left(x_{1}, \ldots, x_{n}\right)$.

Очевидно, для любой теории $T$

$$
\mathrm{EA}+\operatorname{RFN}(T) \vdash \operatorname{Rfn}(T) \text { и } \mathrm{EA}+\operatorname{Rfn}(T) \vdash \operatorname{Con}(T) .
$$

Значит, по теореме Гёделя, ни одна из двух схем реф̆лексии не выводима в $T$ при условии непротиворечивости $T$. Теорема Лёба говорит о том, что, фактически, ни один нетривиальный частньй случай локальной и, тем более, равномерной схемы реф̆лексии не является вьводимьм в $T$. 
Другие естественные формыпринципа рефлексии, как правило, сводятся к одной из этих двух. Например, функция кодирования последовательностей позволяет свернуть несколько свободных переменных в одну, и, таким образом, схема

$$
\forall x\left(\square_{T} \varphi(\dot{x}) \rightarrow \varphi(x)\right)
$$

для формул $\varphi(x)$ с единственной свободной переменной $x$ эквивалентна $\operatorname{RFN}(T)$ над ЕА. С. Феферман [32] установил следующее предложение.

ПРЕДЛОЖЕНИЕ 2.1. Следующие схемы и правила әквивалентны над ЕА:

(a) $\operatorname{RFN}(T)$;

(b) $\forall x \square_{T} \varphi(\dot{x}) \rightarrow \forall x \varphi(x)$;

(c) $\frac{\forall x \square_{T} \varphi(\dot{x})}{\forall x \varphi(x)} \quad$ (правило Клини).

ДокАЗАТЕЛЬСТво. Нам будет полезна следующая лемма [32], назьваемая иногда "явньг принципом рефилексии", поскольку она утверждает доказуемость схемы рефлексии для "стандартных" или “явных" вьводов.

\section{ЛЕмма 2.2.}

(i) Для любой формульи $\varphi(x)$ и любьх $m, n \in \mathbb{N}$

$$
T \vdash \operatorname{Prf}_{T}(\bar{m},\ulcorner\varphi(\bar{n})\urcorner) \rightarrow \varphi(\bar{n})
$$

(ii) Утверждение (i) формализуемо в $\mathrm{EA}$, m.e.

$$
\mathrm{EA} \vdash \forall x, y \quad \square_{T}\left(\operatorname{Prf}_{T}(\dot{y},\ulcorner\varphi(\dot{x})\urcorner) \rightarrow \varphi(\dot{x})\right)
$$

ДокАЗАТЕЛЬство. Достаточно доказать (ii). С одной стороны,

$$
\begin{aligned}
\mathrm{EA} \vdash \operatorname{Prf}_{T}(y,\ulcorner\varphi(\dot{x})\urcorner) & \rightarrow \square_{T} \varphi(\dot{x}) \\
& \rightarrow \square_{T}\left(\operatorname{Prf}_{T}(\dot{y},\ulcorner\varphi(\dot{x})\urcorner) \rightarrow \varphi(\dot{x})\right) .
\end{aligned}
$$

С другой стороны, по доказуемой $\Sigma_{1}$-полноте

$$
\begin{aligned}
\mathrm{EA} \vdash \neg \operatorname{Prf}_{T}(y,\ulcorner\varphi(\dot{x})\urcorner) & \rightarrow \square_{T} \neg \operatorname{Prf}_{T}(\dot{y},\ulcorner\varphi(\dot{x})\urcorner) \\
& \rightarrow \square_{T}\left(\operatorname{Prf}_{T}(\dot{y},\ulcorner\varphi(\dot{x})\urcorner) \rightarrow \varphi(\dot{x})\right) .
\end{aligned}
$$

Комбинируя вместе эти две формулы, получаем требуемое.

Применяя правило Клини (c) к доказуемой формуле из части (ii) предыдущей леммы, мы получаем вывод соответствуюшего частного случая схемы $\operatorname{RFN}(T)$. Таким образом, (c) влечет (a). Импликации (a) $\Rightarrow(\mathrm{b})$ и (b) $\Rightarrow(\mathrm{c})$ очевидны. 
ЗАмечАниЕ 2.3. В отличие от правила Клини, правило локальной рефлексии, т.е. правило

$$
\frac{\square T \varphi}{\varphi}
$$

для предложений $\varphi$, является допустимым в $T$ при условии $\Sigma_{1}$-корректности $T$. Допустимость этого правила выражается формулой

$$
\forall x\left(\square_{T} \square_{T}(\dot{x}) \rightarrow \square_{T}(x)\right),
$$

которая представляет собой частньй случай равномерной схемы рефлексии и тем самым не выводима в $T$. Таким образом, правило локальной рефлексии является допустимьм, но не доказуемо допустимым, в T. Как показал Р. Парих [64], добавление этого правила к $T$ приводит к сушественному ускорению некоторых выводов в $T$, превьшаюшему любую доказуемо тотальную вычислимую функцию.

2.2. Схемы частичной рефлексии. Схемы частичной рефллексии получаются из локальной и равномерной схем наложением ограничения, состоящего в том, что формула $\varphi$ пробегает лишь некоторый подкласс Г класса всех арифметических предложений (формул). Такие схемы обозначаются $\operatorname{Rfn}_{\Gamma}(T)$ и $\operatorname{RFN}_{\Gamma}(T)$ соответственно, а в качестве Г обычно рассматривается один из классов $\Sigma_{n}$ или $\Pi_{n}$ арифметической иерархии.

Лемма 2.4. Следующие схемы әквивалентны над ЕА для любого $n \geqslant 1$ :

(i) $\operatorname{RFN}_{\Sigma_{n}}(T) \equiv \operatorname{RFN}_{\Pi_{n+1}}(T)$;

(ii) $\operatorname{RFN}_{\Pi_{1}}(T) \equiv \operatorname{Rfn}_{\Pi_{1}}(T) \equiv \operatorname{Con}(T)$.

ДоказАтельСтво. (i) Рассмотрим $\Pi_{n+1}$-формулу $\forall y \quad \varphi(y, x)$, где $\varphi(y, x) \in \Sigma_{n}$. Тогда в $\mathrm{EA}+\mathrm{RFN}_{\Sigma_{n}}(T)$ можно, пользуясь свойством $\mathrm{L}^{\circ}$, вьвести

$$
\begin{aligned}
\vdash \square_{T} \forall y \varphi(y, \dot{x}) & \rightarrow \forall y \square_{T} \varphi(\dot{y}, \dot{x}) \\
& \rightarrow \forall y \varphi(y, x) .
\end{aligned}
$$

(ii) Для формулы $\varphi(x) \in \Pi_{1}$, используя доказуемую $\Sigma_{1}$-полноту, вьводим в $\mathrm{EA:}$

$$
\begin{aligned}
\vdash \square_{T} \varphi(\dot{x}) \wedge \neg \varphi(x) & \rightarrow \square_{T} \neg \varphi(\dot{x}) \\
& \rightarrow \square_{T}(\varphi(\dot{x}) \wedge \neg \varphi(\dot{x})) \\
& \rightarrow \square_{T} \perp .
\end{aligned}
$$

Следовательно, $\neg \square_{T} \perp$ влечет $\square_{T} \varphi(\dot{x}) \rightarrow \varphi(x)$.

ЗАмЕчАнИЕ 2.5. Для $n \geqslant 1$ арифметическая сложность всех примеров схемы $\operatorname{RFN}_{\Pi_{n}}(T)$ есть $\Pi_{n}$.

Схемы частичной равномерной рефлексии обладают важным свойством конечной аксиоматизируемости. Как хорошо известно, классы $\Sigma_{n}$ и $\Pi_{n}$-формул имеют определения истинности в теории ЕА [87], [39]. 
ПРЕДЛОЖЕНИЕ 2.6. Для любого $n \geqslant 1$ существует арифметическая $\Pi_{n}$-формула $\operatorname{True}_{\Pi_{n}}(x)$ такая, что для всех $\Pi_{n}$-формул $\varphi\left(x_{1}, \ldots, x_{n}\right)$

$$
\mathrm{EA} \vdash \varphi\left(x_{1}, \ldots, x_{n}\right) \leftrightarrow \operatorname{True}_{\Pi_{n}}\left(\left\ulcorner\varphi\left(\dot{x}_{1}, \ldots, \dot{x}_{n}\right)\right\urcorner\right) .
$$

Аналогично для классов $\Sigma_{n}$.

Формула $\operatorname{True}_{\Pi_{n}}(x)$ называется определением истинности для $\Pi_{n}$-фороул в $\mathrm{EA.}$

Лемма 2.7. Над ЕА схема $\operatorname{RFN}_{\Pi_{n}}(T)$ для $n \geqslant 1$ әквивалентна ее универсальному частному случаю

$$
\forall z \quad\left(\square_{T} \operatorname{True}_{\Pi_{n}}(\dot{z}) \rightarrow \operatorname{True}_{\Pi_{n}}(z)\right)
$$

Аналогичное утверждение имеет место для класса $\Sigma_{n}$.

ДокАЗАТЕльство. Для вывода $\operatorname{RFN}_{\Pi_{n}}(T)$ из формулы (2.1) заметим, что предложение 2.6 влечет

$$
\mathrm{EA} \vdash \forall x_{1} \ldots \forall x_{n} \square_{T}\left(\varphi\left(\dot{x}_{1}, \ldots, \dot{x}_{n}\right) \leftrightarrow \operatorname{True}_{\Pi_{n}}\left(\left\ulcorner\varphi\left(\dot{x}_{1}, \ldots, \dot{x}_{n}\right)\right\urcorner\right)\right),
$$

и, следовательно, можно вьвести

$$
\begin{aligned}
\vdash \square_{T} \varphi\left(\dot{x}_{1}, \ldots, \dot{x}_{n}\right) & \rightarrow \square_{T} \operatorname{Truе}_{\Pi_{n}}\left(\left\ulcorner\varphi\left(\dot{x}_{1}, \ldots, \dot{x}_{n}\right)\right\urcorner\right) \\
& \rightarrow \operatorname{True}_{\Pi_{n}}\left(\left\ulcorner\varphi\left(\dot{x}_{1}, \ldots, \dot{x}_{n}\right)\right\urcorner\right) \quad\left(\text { из }(2.1) \text { с } z=\left\ulcorner\varphi\left(\dot{x}_{1}, \ldots, \dot{x}_{n}\right)\right\urcorner\right) \\
& \rightarrow \varphi\left(x_{1}, \ldots, x_{n}\right) .
\end{aligned}
$$

СлЕДСТВИЕ 2.8. Схемы $\operatorname{RFN}_{\Pi_{n}}(T) u \operatorname{RFN}_{\Sigma_{n}}(T)$ для $n \geqslant 1$ конечно аксиоматизируемь над ЕА.

2.3. $n$-доказуемость и $n$-непротиворечивость. На равномерные схемы частичной рефлексии можно смотреть как на аналоги гёделевской формулы непротиворечивости.

Обозначим множество всех истинных арифметических $\Pi_{n}$-предложений для $n \geqslant 1$ через $\operatorname{Th}_{\Pi_{n}}(\mathbb{N})$. Теория $T$ назьвается $n$-непротиворечивой, если $T+\operatorname{Th}_{\Pi_{n}}(\mathbb{N})$ непротиворечива. $n$-непротиворечивость $T$ может быть выражена следующей формулой, обозначаемой $n$-Con $(T)$ :

$$
\forall z\left(\operatorname{True}_{\Pi_{n}}(z) \rightarrow \neg \square \square_{T} \neg \operatorname{True}_{\Pi_{n}}(\dot{z})\right)
$$

Содержательно эта формулаговорит о совместимости с $T$ любого истинного $\Pi_{n}$-предложения. С точностью до контрапозиции формула $n$-Con $(T)$ совпадает с универсальным частным случаем схемы $\operatorname{RFN}_{\Sigma_{n}}(T)$, т.е. мы имеем следуюшее очевидное следствие леммы 2.7. 
Лемма 2.9. Для $n \geqslant 1$ схема $\operatorname{RFN}_{\Sigma_{n}}(T)$ әквивалентна формуле $n$ - $\operatorname{Con}(T)$ над EA.

Отсюда естественно возникает формула п-доказуемости

$$
[n]_{T} \varphi:=\neg n-\operatorname{Con}(T+\neg \varphi),
$$

выражающая доказуемость $\varphi$ в теории $T+\mathrm{Th}_{\Pi_{n}}(\mathbb{N})$. Заметим, что формула $n-\operatorname{Con}(T)$ имеет сложность $\Pi_{n+1}$, а формула $[n]_{T} \varphi$, тем самым, $\Sigma_{n+1}$. Считаем, что для $n=0$ формулы $n$-доказуемости и $n$-непротиворечивости совпадают с обычными формулами $\square_{T}$ и $\operatorname{Con}(T)$. Мы также будем использовать $\langle n\rangle_{T} \varphi$ как сокращение для $n$ - $\operatorname{Con}(T+\varphi)$.

Свойства формулы $n$-доказуемости аналогичны свойствам обычной формулы доказуемости. Прежде всего, $[n]_{T}$ удовлетворяет условиям Лёба.

ПРЕДЛОЖЕНИЕ 2.10.

$\mathrm{L}^{n} . T \vdash \varphi \Rightarrow \mathrm{EA} \vdash[n]_{T} \varphi$.

$\mathrm{L} 2^{n}$. EA $\vdash[n]_{T}(\varphi \rightarrow \psi) \rightarrow\left([n]_{T} \varphi \rightarrow[n]_{T} \psi\right)$.

$\mathrm{L}^{n}{ }^{n}$. EA $\vdash[n]_{T} \varphi \rightarrow[n]_{T}[n]_{T} \varphi$.

Аналогичные утверждения также имеют место для формул со свободными переменными.

ДокАЗАТЕЛЬСТво. Первые два условия легко проверяются с учетом того, что

$$
\mathrm{EA} \vdash[n]_{T} \varphi \leftrightarrow \exists z\left(\operatorname{True}_{\Pi_{n}}(z) \wedge \square_{T}\left(\operatorname{True}_{\Pi_{n}}(\dot{z}) \rightarrow \varphi\right) .\right.
$$

Третье условие вытекает из более общего факта доказуемой $\Sigma_{n+1}$-nолноты.

ПРЕДЛОЖЕНИЕ 2.11. Для любой $\Sigma_{n+1}$ - бормуль $\sigma\left(x_{1}, \ldots, x_{k}\right)$, где все свободнье переменные б указаны явно,

$$
\mathrm{EA} \vdash \sigma\left(x_{1}, \ldots, x_{k}\right) \rightarrow[n]_{T} \sigma\left(\dot{x}_{1}, \ldots, \dot{x}_{k}\right) .
$$

ДоказАТЕЛьСтво. Пусть $\sigma=\exists x \sigma_{0}(x)$, где $\sigma_{0} \in \Pi_{n}$. Тогда мы имеем, пренебрегая остальными свободными переменньми,

$$
\mathrm{EA} \vdash \sigma_{0}(x) \leftrightarrow \operatorname{True}_{\Pi_{n}}\left(\left\ulcorner\sigma_{0}(\dot{x})\right\urcorner\right),
$$

а также

$$
\mathrm{EA} \vdash \square_{T}\left(\sigma_{0}(\dot{x}) \leftrightarrow \operatorname{True}_{\Pi_{n}}\left(\left\ulcorner\sigma_{0}(\dot{x})\right\urcorner\right)\right) .
$$

Поэтому

$$
\mathrm{EA} \vdash \sigma_{0}(x) \rightarrow \exists z\left(\operatorname{True}_{\Pi_{n}}(z) \wedge \square_{T}\left(\operatorname{True}_{\Pi_{n}}(\dot{z}) \rightarrow \sigma_{0}(\dot{x})\right)\right),
$$

где в качестве $z$ можно взять значение терма $\left\ulcorner\sigma_{0}(\dot{x})\right\urcorner$. То есть

$$
\begin{aligned}
\mathrm{EA} \vdash \sigma_{0}(x) & \rightarrow[n]_{T} \sigma_{0}(\dot{x}), \\
& \rightarrow[n]_{T} \exists x \sigma_{0}(x) \\
& \rightarrow[n]_{T} \sigma .
\end{aligned}
$$

Отсюда $\mathrm{EA} \vdash \exists x \sigma_{0}(x) \rightarrow[n]_{T} \sigma$.

Как стандартное следствие условий Лёба и леммы о неподвижной точке мы получаем аналог формализованной теоремы Лёба. 
ПРЕДЛОЖЕНИЕ 2.12. Для любой формульи $\varphi$

$$
\mathrm{EA} \vdash[n]_{T}\left([n]_{T} \varphi \rightarrow \varphi\right) \rightarrow[n]_{T} \varphi
$$

На формулу $n$-доказуемости можно также перенести понятие логики доказуемости. Рассмотрим язык логики высказываний с модальностью $[n]$ и обозначим через $\mathbf{G L}^{n}$ вариант логики GL в этом языке. Арифметическая интерпретация $(\varphi)_{T}^{*}$ модальной формулы $\varphi$ определяется как обычно, с той разницей, что $[n]$ расшифровьвается как $n$-доказуемость в теории $T$ :

$$
([n] \varphi)_{T}^{*}=[n]_{T}(\varphi)_{T}^{*}
$$

Из предложений 2.10 и 2.12 вытекает следуюшее утверждение.

СлЕДСТВИЕ 2.13. Если GL $\mathbf{G}^{n} \vdash \varphi$, то EA $\vdash(\varphi)_{T}^{*}$ для любой реализации $(\cdot)^{*}$ переменных $\varphi$.

Фактически, как показал К. Сморинский [87], на логику $n$-доказуемости переносится без больших изменений и теорема Соловея.

ПРЕДЛОЖЕНИЕ 2.14. Пусть элементарно аксиоматизированная теория $T$ $\Sigma_{n+1}$-корректна и содерәит ЕА. Тогда

$$
\mathbf{G L}^{n} \vdash \varphi \Longleftrightarrow T \vdash(\varphi)_{T}^{*} \text { для любой реализации }(\cdot)^{*} \text { переменньх } \varphi \text {. }
$$

ЗАмЕчАНИЕ 2.15. Понятие $n$-непротиворечивости играет в данной работе большую роль. Отметим, что Гёдель рассматривал родственное, но не эквивалентное понятие $\omega$-непротиворечивости. Теория $T \omega$-противоречива, если для некоторой формулы $\varphi(x)$ одновременно имеют место условия:

(i) $T \vdash \exists x \varphi(x)$;

(ii) $T \vdash \neg \varphi(0), \neg \varphi(1), \neg \varphi(2), \ldots$

В противном случае $T$ называется $\omega$-непротиворечивой.

В современных изложениях теорем Гёделя условие $\omega$-непротиворечивости теории часто заменяется на более слабое условие $\Sigma_{1}$-корректности. К. Сморинский [85] показал, что формула, естественным образом выражающая $\omega$-непротиворечивость $T$, эквивалентна $\operatorname{RFN}_{\Pi_{3}}(T+\mathrm{RFN}(T))$. Таким образом, формула $\omega$-непротиворечивости выражается через более удобные схемы рефлексии.

2.4. Теоремы о неограниченности. Многие приложения схем рефилексии для анализа формальных теорий основьваются на так называемых теоремах о неограниченности, принадлежаших Крайзелю и Леви.

Говорят, что теория $U$ есть конечное расширение теории $T$, если она аксиоматизируема над $T$ конечным числом предложений. $U$ назьвается расширением $T$ арифметической сложности Г или Г-расширением $T$, если $U$ аксиоматизируема над $T$ множеством предложений из класса Г. В качестве Г обычно рассматривается один из классов арифметической иерархии. (Заметим, что, в приншипе, множество гёделевых номеров аксиом $U$ может не быть перечислимьм или даже арифметическим. Речь идет о сложности формул, аксиоматизирующих $U$.) Теория $U$ называется $\Gamma$-консервативной над $T$, если для любого предложени $\varphi \in \Gamma U \vdash \varphi$ влечет $T \vdash \varphi$ (обозначение $\left.U \subseteq_{\Gamma} T\right)$. 
ПРЕДЛОЖЕНИЕ 2.16. Пусть Т - әлементарно аксиоматизированная теория.

(i) $\operatorname{Rfn}_{\Pi_{n}}(T)$ не содерәстся ни в одном непротиворечивом конечном расширении T сложности $\Sigma_{n}$ ( и двойственно для $\left.\operatorname{Rfn}_{\Sigma_{n}}\right)$.

(ii) $\operatorname{RFN}_{\Pi_{n}}(T)$ не содержится ни в одном непротиворечивом расширении $T$ сложности $\Sigma_{n}$.

ДокаЗАТЕльство. (i) Допустим $T+\varphi \vdash \operatorname{Rfn}_{\Pi_{n}}(T)$, где $\varphi \in \Sigma_{n}$. Тогда, в частности,

$$
T+\varphi \vdash \square_{T} \neg \varphi \rightarrow \neg \varphi
$$

Поэтому

$$
\begin{aligned}
& T \vdash \varphi \rightarrow\left(\square_{T} \neg \varphi \rightarrow \neg \varphi\right), \\
& \quad \vdash \square_{T} \neg \varphi \rightarrow \neg \varphi .
\end{aligned}
$$

Отсюда по теореме Лёба $T \vdash \neg \varphi$, т.е. теория $T+\varphi$ противоречива.

(ii) Допустим, что $U$ - расширение $T$ сложности $\Sigma_{n}$ такое, что $U \vdash \operatorname{RFN}_{\Pi_{n}}(T)$. Поскольку схема $\operatorname{RFN}_{\Pi_{n}}(T)$ конечно аксиоматизируема, сушествует конечная подтеория $U_{0} \subseteq U$ такая, что $U_{0} \vdash \mathrm{RFN}_{\Pi_{n}}(T)$. Но тогда $U_{0}$ также содержит $\operatorname{Rfn}_{\Pi_{n}}(T)$ и в силу (i) $T+U_{0}$ является противоречивой. Следовательно, такова и теория $U$.

СлЕДСТВИЕ 2.17. Пусть Т - элементарно аксиоматизированная теория.

(i) $\operatorname{Rfn}(T)$ не содержится ни в одном конечном непротиворечивом расиирении $T$.

(ii) $\operatorname{RFN}(T)$ не содержится ни в одном непротиворечивом расиирении $T$ ограниченной арифметической сложности.

ПримеР 2.18. Согласно теореме Крайзеля-Леви, арифметика Пеано РА доказывает RFN(EA). Следовательно, РА не только не является конечно аксиоматизируемой теорией, но и не содержится ни в каком расширении ЕА ограниченной арифметической сложности.

СлЕДСТВИЕ 2.19. Если теория $T \quad \Sigma_{1}$-корректна, то

$$
T+\operatorname{Rfn}(T) \nvdash \operatorname{RFN}(T) .
$$

ДоказАТЕЛЬство. Пусть $U$ - расширение $T$ множеством всех истинных $\Pi_{1}$-предложений. (Теория $U$ неперечислима, но является П 1 -расширением $T$.) Поскольку $T$ не доказывает ложных $\Sigma_{1}$-предложений, $U$ непротиворечива. По предыдушему следствию $U \nvdash \operatorname{RFN}(T)$. С другой стороны, $U \vdash \operatorname{Rfn}(T)$, поскольку

$$
\begin{aligned}
T \vdash \varphi & \Rightarrow T \vdash \square_{T} \varphi \rightarrow \varphi \text { и } \\
T \nvdash \varphi & \Rightarrow \mathbb{N} \vDash \neg \square_{T} \varphi \Rightarrow \\
\Rightarrow U \vdash \neg \square T \varphi & \Rightarrow U \vdash \square_{T} \varphi \rightarrow \varphi .
\end{aligned}
$$


ЗАмечАниЕ 2.20. Условие $\Sigma_{1}$-корректности в этом известном утверждении может быть ослаблено, однако это требует более глубоких соображений (см. следствие 2.38 ниже).

Часть (i) предложения 2.16 и следствия 2.17 имеет важное усиление. Слово "конечное" в них может быть заменено на "рекурсивно перечислимое". Это вытекает из следующего результата.

Лемма 2.21. Пусть $U$-непротиворечивое перечислимое расиирение $T$ сложности $\Sigma_{n}$. Тогда найдется $\Sigma_{n}$-предложение $\varphi$ такое, что $T+\varphi$ непротиворечива и содержит $U$. (Двойственно для $\Pi_{n}$-аксиоматизированных расширений.)

ДокАЗАТЕльство. Для теорий, содержаших схему $\Sigma_{1}$-подстановки, в частности, для РА, результат вытекает непосредственно из теоремы 4 работы П. Линдстрема [52]. Однако для более слабых теорий требуется модификация этой конструкции, которую мы приводим ниже.

По лемме Крейга можно считать, что множество гёделевых номеров $\Sigma_{n}$-аксиом теории $U$ над $T$ задается некоторой элементарной формулой $\mathrm{Ax}_{U}(x)$. Кроме того, определение истинности для $\Sigma_{n}$-форорул имеет вид

$$
\mathrm{EA} \vdash \operatorname{True}_{\Sigma_{n}}(x) \leftrightarrow \exists z \operatorname{Wit}_{\Sigma_{n}}(z, x)
$$

для некоторой формулы Wit $_{\Sigma_{n}}(z, x) \in \Pi_{n-1}$. Рассмотрим неподвижную точку $\varphi$ такую, что

$$
\mathrm{EA} \vdash \varphi \leftrightarrow \exists x \exists z\left(\forall y \leqslant x\left(\operatorname{Ax}_{U}(y) \rightarrow \operatorname{Wit}_{\Sigma_{n}}\left((z)_{y}, y\right)\right) \wedge \operatorname{Prf}_{T}(x,\ulcorner\neg \varphi\urcorner)\right) .
$$

Мы утверждаем, что $\varphi$ удовлетворяет условиям леммы.

1) Теория $T+\varphi$ непротиворечива. Действительно, в противном случае найдется число $m$ такое, что

$$
\mathrm{EA} \vdash \operatorname{Prf}_{T}(\bar{m},\ulcorner\neg \varphi\urcorner) .
$$

С другой стороны, для каждой аксиомы $\sigma \in \Sigma_{n}$ теории $U$ такой, что $\ulcorner\sigma\urcorner=y \leqslant m$, мы имеем $U \vdash \exists z_{y}$ Wit $_{\Sigma_{n}}(z y, \bar{y})$. Поскольку для данного $m$ таких формул $\sigma$ сушествует лишь конечное число, все соответствуюшие $z_{y}$ можно собрать в одну последовательность $z:=\left\langle z_{0}, \ldots, z_{m}\right\rangle$, где $z_{y}:=0$ для чисел $y$, не являюшихся кодами аксиом $U$. Таким образом,

$$
U \vdash \exists z \bigwedge_{y \leqslant m, y \in \mathrm{Ax}_{U}} \operatorname{Wit}_{\Sigma_{n}}\left((z)_{\bar{y}}, \bar{y}\right),
$$

откуда вытекает, что

$$
U \vdash \exists z \forall y \leqslant \bar{m}\left(\operatorname{Ax}_{U}(y) \rightarrow \operatorname{Wit}_{\Sigma_{n}}\left((z)_{y}, y\right)\right) .
$$

Следовательно, $U \vdash \varphi$ и $U \vdash \perp$, поскольку по нашему предположению теория $T+\varphi$ противоречива.

2) $T+\varphi$ содержит $U$. Рассмотрим любую формулу $\sigma \in \mathrm{Ax}_{U},\ulcorner\sigma\urcorner=m$. Прежде всего заметим, что по явному принщипу реффлексии

$$
T+\varphi \vdash \forall x \leqslant \bar{m} \neg \operatorname{Prf}_{T}(x,\ulcorner\neg \varphi\urcorner) .
$$


Отсюда, рассуждая в теории $T+\varphi$, вьводим:

$$
\begin{aligned}
\vdash \forall y \leqslant x\left(\operatorname{Ax}_{U}(x) \rightarrow \operatorname{Wit}_{\Sigma_{n}}\left((z)_{y}, y\right)\right) \wedge \operatorname{Prf}_{T}(x,\ulcorner\neg \varphi\urcorner) & \rightarrow \bar{m} \leqslant x \wedge \operatorname{Ax}_{U}(\bar{m}) \\
& \rightarrow \operatorname{Wit}_{\Sigma_{n}}((z) \bar{m}, \bar{m}) \\
& \rightarrow \operatorname{True}_{\Sigma_{n}}(\bar{m}) \\
& \rightarrow \sigma .
\end{aligned}
$$

Отсюда следует, что $T+\varphi \vdash \sigma$.

СЛЕДСТВИЕ 2.22.

(i) $\operatorname{Rfn}_{\Pi_{n}}(T)$ не содержится ни в одном непротиворечивом перечислимом расширении $T$ сложности $\Sigma_{n}$ (и двойственно для $\left.\operatorname{Rfn}_{\Sigma_{n}}(T)\right)$.

(ii) $\operatorname{Rfn}(T)$ не содержится ни в одном непротиворечивом перечислимом расширении Т ограниченной арифметической сложности.

2.5. Иерархии схем частичной рефлексии. В этом пункте мы рассматриваем подробно иерархии схем частичной рефлексии. Мы показьваем, что свойства схем локальной рефлексии контрастируют с хорошо известными свойствами схем частичной равномерной рефлексии. Из п. 2.1 известны следующие факты.

1) Схемы частичной равномерной рефлексии для фиксированной элементарно аксиоматизированной теории $T$ составляют линейно упорядоченную по включению иерархию (см. рис. 1).

2) За исключением полной равномерной схемы реффлексии $\operatorname{RFN}(T)$ все схемы в этой иерархии конечно аксиоматизируемы (следствие 2.8).

Следующая лемма показьвает, что в определенном смысле схема $\operatorname{RFN}_{\Pi_{n+1}}(T)$ много сильнее $\operatorname{RFN}_{\Pi_{n}}(T)$.

Лемма 2.23. Для любого конечного $\Sigma_{n}$-расширения $U$ теории $T$ схема $\operatorname{RFN}_{\Pi_{n}}(T)$ дедуктивно әквивалентна $\operatorname{RFN}_{\Pi_{n}}(U)$ надU.

ДокаЗАТЕЛЬство. Достаточно вывести $\operatorname{RFN}_{\Pi_{n}}(U)$ в $U+\operatorname{RFN}_{\Pi_{n}}(T)$. С использованием формализованной теоремы о дедукщии для любой $\Pi_{n}$-формулы $\varphi(x)$ в $\mathrm{EA}+$ $\operatorname{RFN}_{\Pi_{n}}(T)$ вьводимо:

$$
\begin{aligned}
\vdash \square_{U} \varphi(\dot{x}) & \rightarrow \square_{T}(\psi \rightarrow \varphi(\dot{x})) \\
& \rightarrow(\psi \rightarrow \varphi(x)),
\end{aligned}
$$

где $\psi$ означает конъюнкцию всех $\Sigma_{n}$-аксиом теории $U$. Поскольку $\psi$ вьводима в $U$, получаем

$$
U+\operatorname{RFN}_{\Pi_{n}}(T) \vdash \square U \varphi(\dot{x}) \rightarrow \varphi(x) .
$$

СлЕДСТВИЕ 2.24. В теории $T+\operatorname{RFN}_{\Pi_{n+1}}(T)$ доказуемо $\operatorname{Con}\left(T+\operatorname{RFN}_{\Pi_{n}}(T)\right)$.

Таким образом, иерархия схем частичной равномерной рефлексии не вырождается (все теории иерархии различны), если и только если теория $T+\operatorname{RFN}(T)$ непротиворечива. Более того, в случае, когда иерархия невырождена, ни одно из расширений на любом уровне иерархии не является консервативным даже для $\Pi_{1}$-предложений. 


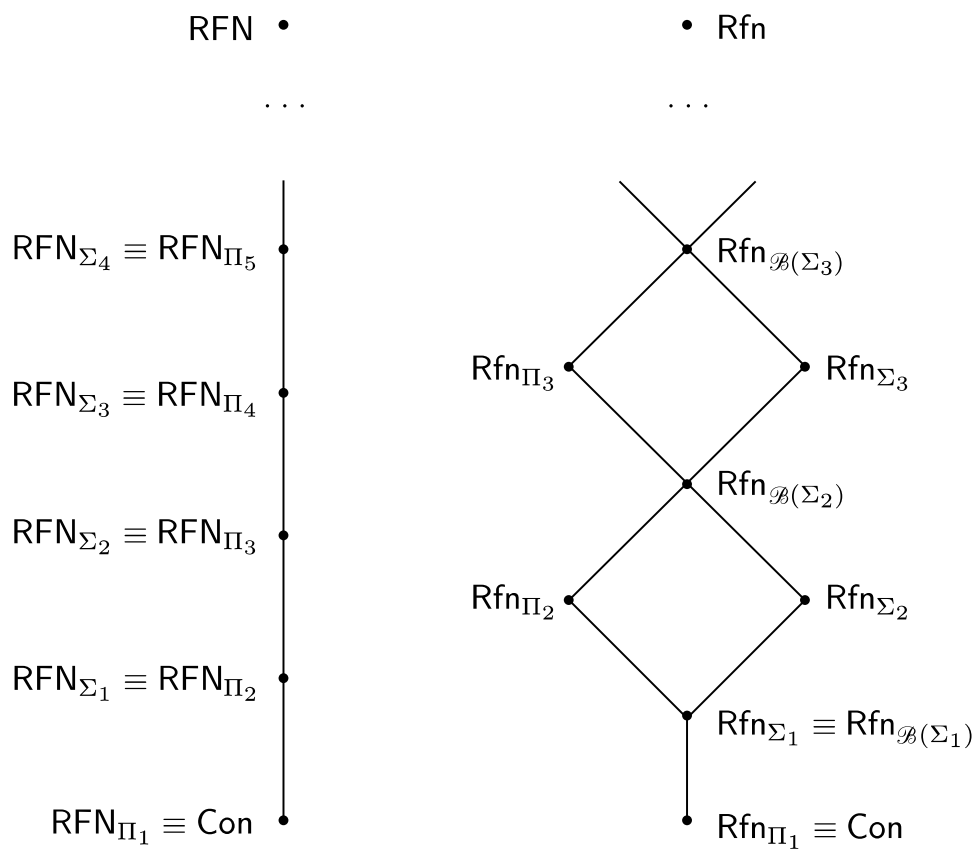

Рис. 1. Иерархии схем частичной рефолексии

Для иерархии схем частичной локальной рефлексии картина существенно меняется. Во-первых, имеет место следующее утверждение.

ПРЕДЛОЖЕНИЕ 2.25. Все схемы $\operatorname{Rfn}_{\Sigma_{n}}(T)$ u $\operatorname{Rfn}_{\Pi_{n}}(T)$ попарно неәквивалентны при условии непротиворечивости теории $T+\operatorname{Rfn}(T)$. В частности, $\operatorname{Rfn}_{\Sigma_{n}}(T)$ строго включена в $\operatorname{Rfn}_{\Pi_{n+1}}(T)$ и ни одна из схем $\operatorname{Rfn}_{\Sigma_{n}}(T)$ и $\operatorname{Rfn}_{\Pi_{n}}(T)$ не влечет другую для любого $n>1$ (см. puс. 1$)$.

ДокАЗАТЕЛЬСТво. Поскольку в силу первой части предложения 2.16 схема $\operatorname{Rfn}_{\Pi_{1}}(T)$, эквивалентная $\Pi_{1}$-предложению $\operatorname{Con}(T)$, строго слабее $\operatorname{Rfn}_{\Sigma_{1}}(T)$, нам будет достаточно установить лишш второе утверждение леммы.

Заметим, что для $n>1 \operatorname{Rfn}_{\Sigma_{n}}(T)$ есть перечислимое, и даже элементарное, множество $\Sigma_{n}$-предложений. Таким образом, по следствию 2.22 ,

$$
T+\operatorname{Rfn}_{\Sigma_{n}}(T) \nvdash \operatorname{Rfn}_{\Pi_{n}}(T) .
$$

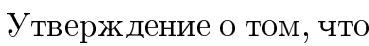

$$
T+\operatorname{Rfn}_{\Pi_{n}}(T) \nvdash \operatorname{Rfn}_{\Sigma_{n}}(T),
$$

доказывается симметричным образом.

Пусть $\mathscr{B}\left(\Sigma_{n}\right)$ обозначает множество всех булевых комбинаций $\Sigma_{n}$-предложений. Мы имеем следующее полезное утверждение.

Лемма 2.26. Над ЕА схема $\operatorname{Rfn}_{\Sigma_{1}}(T)$ әквивалентна $\operatorname{Rfn}_{\mathscr{B}\left(\Sigma_{1}\right)}(T)$. 
ДокАЗАТЕЛЬСТво. Докажем, что

$$
\mathrm{EA}+\operatorname{Rfn}_{\Sigma_{1}}(T) \vdash \square_{T} \varphi \rightarrow \varphi
$$

для любой булевой комбинации $\Sigma_{1}$-предложений $\varphi$. Формула $\varphi$ логически эквивалентна формуле вида $\bigwedge_{i=1}^{n}\left(\pi_{i} \vee \sigma_{i}\right)$ для некоторых предложений $\pi_{i} \in \Pi_{1}$ и $\sigma_{i} \in \Sigma_{1}$. Поскольку предикат доказуемости $\square_{T}$ коммутирует с конъюнкцией, достаточно вывести в $\mathrm{EA}+\mathrm{Rfn}_{\Sigma_{1}}(T)$ формулы

$$
\operatorname{Prov}_{T}\left(\left\ulcorner\left(\pi_{i} \vee \sigma_{i}\right)\right\urcorner\right) \rightarrow\left(\pi_{i} \vee \sigma_{i}\right)
$$

для любого $i$. Опираясь на доказуемую $\Sigma_{1}$-полноту и условия Лёба, получаем

$$
\begin{aligned}
\vdash \operatorname{Prov}_{T}\left(\left\ulcorner\left(\pi_{i} \vee \sigma_{i}\right)\right\urcorner\right) \wedge \neg \pi_{i} & \rightarrow \operatorname{Prov}_{T}\left(\left\ulcorner\neg \pi_{i}\right\urcorner\right) \\
& \rightarrow \operatorname{Prov}_{T}\left(\left\ulcorner\sigma_{i}\right\urcorner\right) \\
& \rightarrow \sigma_{i},
\end{aligned}
$$

используя $\operatorname{Rfn}_{\Sigma_{1}}(T)$. Отсюда

$$
\mathrm{EA}+\operatorname{Rfn}_{\Sigma_{1}}(T) \vdash \operatorname{Prov}_{T}\left(\left\ulcorner\left(\pi_{i} \vee \sigma_{i}\right)\right\urcorner\right) \rightarrow\left(\pi_{i} \vee \sigma_{i}\right)
$$

Отметим, что в силу предложения 2.25 прямое обобщение этого утверждения на классы $\Sigma_{n}$ при $n>1$ не имеет места. (Некоторое разумное обобщение, использующее релятивизированные схемы рефлексии, приведено в п. 4.4.)

Далее, для схем локальной рефлексии мы имеем следуюшие результаты.

1) Ни одна из схем $\operatorname{Rfn}_{\Sigma_{n}}(T)$ и $\operatorname{Rfn}_{\Pi_{n+1}}(T)$ для $n \geqslant 1$ не является конечно аксиоматизируемой над $T$. (Единственным исключением является схема $\operatorname{Rfn}_{\Pi_{1}}(T)$, которая эквивалентна $\operatorname{Con}(T)$.)

2) Все схемы локальной рефилексии, за очевидньм исключением $\operatorname{Rfn}_{\Pi_{1}}(T)$, являются П $\Pi_{1}$-консервативными друг над другом, т.е. доказьвают одни и те же $\Pi_{1}$-предложения. В частности, в теории $T+\operatorname{Rfn}_{\Pi_{n+1}}(T)$ не выводима непротиворечивость $T+\operatorname{Rfn}_{\Pi_{n}}(T)$ для $n>1[38]$.

3) Имеет место более сильное утверждение [15]: полная схема локальной рефиексии $\operatorname{Rfn}(T) \Pi_{n^{-}}$(соответственно $\Sigma_{n^{-}}$) консервативна над $T+\operatorname{Rfn}_{\Pi_{n}}(T)$ (соответственно $\left.T+\operatorname{Rfn}_{\Sigma_{n}}(T)\right)$. Этот результат о консервативности оптимален относительно ариф̆метической сложности в том смысле, что схемы можно различить уже на уровне $\Sigma_{n^{-}}$ (соответственно $\Pi_{n^{-}}$) предложений.

Доказательства этих утверждений представляют собой красивое применение техники логики доказуемости. Первьй результат этого типа был найден Дж. Булосом [22] и С.Н. Артемовым [4], [5], которые независимо показали, что $n$-кратно итерированная формула непротиворечивости для корректной теории $T$ не может быть вьведена из менее чем $n$ частных случаев локальной схемы рефлексии. Мы получим вышеприведенные утверждения с помошью единой более общей конструкции, найденной в [15]. Эти результаты будут затем применены к исследованию схем беспараметрической индукции в арифметике. 
Лемма 2.27. Определим модальные формуль $Q_{i}$ следующим образом:

$$
Q_{1}:=p, \quad Q_{i+1}:=Q_{i} \vee \square Q_{i}
$$

где $p$ - пропозичиональная переменная. Тогда для любых переменных $p_{1}, \ldots, p_{m}$

$$
\mathbf{G L} \vdash \square^{+}\left(\bigwedge_{i=1}^{m}\left(\square p_{i} \rightarrow p_{i}\right) \rightarrow p\right) \rightarrow\left(\bigwedge_{i=1}^{m}\left(\square Q_{i} \rightarrow Q_{i}\right) \rightarrow p\right)
$$

ДокАЗАТЕльство. Не предъявляя явно доказательства формулы (2.2), мы установим ее выводимость в GL, опираясь на стандартную семантику Крипке логики GL.

Рассмотрим любую модель Крипке $(W, R, \Vdash)$, в которой заключение $\left(\bigwedge_{i=1}^{m}\left(\square Q_{i} \rightarrow\right.\right.$ $\left.Q_{i}\right) \rightarrow p$ ) ложно в некотором мире $x \in W$. Это означает, что $x \nVdash p$ и $x \Vdash \square Q_{i} \rightarrow Q_{i}$ для каждого $i \leqslant m$. С помощью очевидной индукщии по $i$ нетрудно установить, что $x \nVdash Q_{i}$ для всех $i \leqslant m+1$, в частности, $x \nVdash Q_{m+1}$.

Отсюда, расшифровывая определение $Q_{m+1}$, мы видим, что в $W$ найдется последовательность миров

$$
x=x_{m+1} R x_{m} R \ldots x_{2} R x_{1}
$$

такая, что $x_{i} \nVdash Q_{i}$ для всех $i \leqslant m+1$. Поскольку отношение $R$ иррефолексивно и транзитивно, все $x_{i}$ попарно различны. Кроме того, индукцией по $i$ нетрудно убедиться, что для всех $i$

$$
\mathbf{G L} \vdash p \rightarrow Q_{i}
$$

Значит, $x_{i} \nVdash p$ для любого $i \leqslant m+1$.

Заметим теперь, что для любого $i$ формула $\square p_{i} \rightarrow p_{i}$ может быть ложной не более чем в одном элементе линейной цепи $x_{m+1}, \ldots, x_{1}$. Отсюда, по принципу Дирихле, среди $m+1$ мира $x_{i}$ найдется мир $z$ такой, что

$$
z \Vdash \bigwedge_{i=1}^{m}\left(\square p_{i} \rightarrow p_{i}\right) \wedge \neg p
$$

В случае, если $z$ совпадает с $x=x_{m+1}$, мы имеем

$$
x \nVdash \bigwedge_{i=1}^{m}\left(\square p_{i} \rightarrow p_{i}\right) \rightarrow p .
$$

В случае, когда $z=x_{i}$ для некоторого $i \leqslant m$, мы имеем $x R z$ по транзитивности $R$, и, таким образом,

$$
x \nVdash \square\left(\bigwedge_{i=1}^{m}\left(\square p_{i} \rightarrow p_{i}\right) \rightarrow p\right) .
$$

Это показывает, что формула (2.2) вьнуждается в любом мире любой модели Крипке; следовательно, она вьводима в GL.

СлЕДСТВИЕ 2.28. GL $\vdash \square^{+} \neg \bigwedge_{i=1}^{m}\left(\square p_{i} \rightarrow p_{i}\right) \rightarrow \square^{m} \perp$. 
ДоКАЗАТЕЛЬСТво. Подставляя в (2.2) формулу $\perp$ вместо $p$, получаем

$$
\mathbf{G L} \vdash Q_{i}(p / \perp) \leftrightarrow \square^{i-1} \perp
$$

а значит,

$$
\mathbf{G L} \vdash \bigwedge_{i=1}^{m}\left(\square Q_{i}(p / \perp) \rightarrow Q_{i}(p / \perp)\right) \leftrightarrow \neg \square^{m} \perp
$$

Прогрессия теорий, основанная на итерации формуль непротиворечивости, определяется следуюшим образом:

$$
T_{0}:=T, \quad T_{n+1}:=T_{n}+\operatorname{Con}\left(T_{n}\right), \quad T_{\omega}:=\bigcup_{n \geqslant 0} T_{n}
$$

Лемма 2.29. EA $\vdash \operatorname{Con}\left(T_{n}\right) \leftrightarrow \neg \square_{T}^{n+1} \perp$.

ДокАЗАТЕЛЬСТВо. Индукция по $n$ с использованием условий Лёба.

Формула $\operatorname{Con}\left(T_{n}\right)$ назьвается $n$-кратно итерированной формулой непротиворечивости теории $T$. Если $T-\Sigma_{1}$-корректная теория, то по теореме Гёделя $T_{n}$ образуют строго возрастаюшую последовательность $\Sigma_{1}$-корректных расширений $T$.

Следующее утверждение получено в [22], [5].

ПРЕДЛОЖЕНИЕ 2.30. Con $\left(T_{n}\right)$ не выводима ни из каких $n$ частных случаев локальной схемы рефлексии для теории $T$, при условии непротиворечивости $T_{n}$.

ДокаЗАТЕльство. Допустим

$$
T \vdash \bigwedge_{i=1}^{n}\left(\square_{T} \varphi_{i} \rightarrow \varphi_{i}\right) \rightarrow \operatorname{Con}\left(T_{n}\right) .
$$

Тогда по лемме 2.29, с помошью контрапозиции,

$$
T \vdash \square_{T}^{n+1} \perp \rightarrow \neg \bigwedge_{i=1}^{n}\left(\square_{T} \varphi_{i} \rightarrow \varphi_{i}\right),
$$

и, используя условия Лёба, получаем

$$
\left.\mathrm{EA} \vdash \square_{T}^{n+2} \perp \rightarrow \square_{T}\right\urcorner \bigwedge_{i=1}^{n}\left(\square_{T} \varphi_{i} \rightarrow \varphi_{i}\right)
$$

Рассматривая арифметическую реализацию, сопоставляющую каждой переменной $p_{i}$ формулу $\varphi_{i}$, по следствию 2.28 получаем

$$
\mathrm{EA} \vdash \square_{T}^{n+2} \perp \rightarrow \square_{T}^{n+1} \perp
$$

Отсюда по теореме Лёба $\mathrm{EA} \vdash \square_{T}^{n+1} \perp$, а значит, теория $T_{n}$ противоречива. 
ЗАмечАниЕ 2.31. Формулы $\square_{T}^{i+1} \perp \rightarrow \square_{T}^{i} \perp$ для $i \leqslant n$ дают пример $(n+1)$-го частного случая локальной схемы рефлексии (фактически, схемы $\operatorname{Rfn}_{\Sigma_{1}}(T)$ ), из которых вьводима формула $\operatorname{Con}\left(T_{n}\right)$ над ЕА. Заметим также, что отсюда следует включение $T_{\omega} \subseteq T+\operatorname{Rfn}_{\Sigma_{1}}(T)$.

ПРЕДЛОЖЕНИЕ 2.32. Для $n \geqslant 1$ схемы $\operatorname{Rfn}_{\Sigma_{n}}(T) u \operatorname{Rfn}_{\Pi_{n+1}}(T)$ не являются конечно аксиоматизируемыми надт при условии непротиворечивости теории $T_{\omega}$.

ДоКАЗАТЕЛЬСТВо. По предложению 2.30 никаких $m$ примеров этих схем не достаточно, чтобы вывести формулу $\operatorname{Con}\left(T_{m}\right)$. С другой стороны, формула $\operatorname{Con}\left(T_{m}\right)$ вьводима в $T_{\omega}$, а значит, и в любой из схем $\operatorname{Rfn}_{\Sigma_{n}}(T)$ и $\operatorname{Rfn}_{\Pi_{n+1}}(T)$ при $n \geqslant 1$.

Другим выражением той же самой идеи является следуюший результат, полученный С. В. Горячевым в [38].

ПРЕДЛОЖЕНИЕ 2.33. Любые $n$ частных случаев локальной схемы рефлексии для $T \Pi_{1}$-консервативны над теорией $T_{n}$.

ДОКАЗАТЕЛЬСТВО. Пусть $\pi \in \Pi_{1}$ и

$$
T \vdash \bigwedge_{i=1}^{n}\left(\square_{T} \varphi_{i} \rightarrow \varphi_{i}\right) \rightarrow \pi .
$$

Тогда в силу условий Лёба

$$
T \vdash \square_{T} \neg \pi \rightarrow \square_{T} \neg \bigwedge_{i=1}^{n}\left(\square_{T} \varphi_{i} \rightarrow \varphi_{i}\right)
$$

и

$$
T \vdash \square_{T}^{+} \neg \pi \rightarrow \square^{+} \neg \bigwedge_{i=1}^{n}\left(\square_{T} \varphi_{i} \rightarrow \varphi_{i}\right) .
$$

С помошњю арифметической интерпретации из следствия 2.28 мы получаем

$$
T \vdash \square_{T}^{+} \neg \pi \rightarrow \square_{T}^{n} \perp
$$

откуда

$$
T \vdash \neg \pi \rightarrow \square_{T}^{n} \perp
$$

по доказуемой $\Sigma_{1}$-полноте. Таким образом, $T \vdash \neg \square_{T}^{n} \perp \rightarrow \pi$ и $T_{n} \vdash \pi$.

СлЕДСТВИЕ 2.34. Для любой әлементарно аксиоматизированной теории Т в теориях $T+\operatorname{Rfn}(T)$ и $T_{\omega}$ выводимы одни и те же $\Pi_{1}$-предложения.

Отсюда также получается следующее утверждение.

СлеДСТВИЕ 2.35. Теория $T+\operatorname{Rfn}(T)$ непротиворечива тогда и только тогда, когда непротиворечива теория $T_{\omega}$.

Как уже отмечалось, $T+\operatorname{Rfn}_{\Sigma_{n}}(T)$ содержит $T_{\omega}$ для любого $n \geqslant 1$. Следовательно, в отличие от схем равномерной рефлексии, все такие схемы доказывают одни и те же $\Pi_{1}$-предложения. Фактически, для схем частичной локальной рефлексии имеет место более сильньй результат, установленный в [15]. 
Теорема 6. Для $n>1$ над T имеют место следующие результаты о консервативности:

(i) $\operatorname{Rfn}(T) \subseteq_{\Pi_{n}} \operatorname{Rfn}_{\Pi_{n}}(T)$;

(ii) $\operatorname{Rfn}(T) \subseteq \subseteq_{n} \operatorname{Rfn}_{\Sigma_{n}}(T)$;

(iii) $\operatorname{Rfn}(T) \subseteq \mathscr{B}\left(\Sigma_{1}\right) \operatorname{Rfn}_{\Sigma_{1}}(T)$.

ДоКАЗАТЕЛЬСТво. Допустим

$$
T \vdash \bigwedge_{i=1}^{m}\left(\square_{T} \varphi_{i} \rightarrow \varphi_{i}\right) \rightarrow \pi .
$$

В силу условий Лёба мы также имеем

$$
T \vdash \square_{T}\left(\bigwedge_{i=1}^{m}\left(\square_{T} \varphi_{i} \rightarrow \varphi_{i}\right) \rightarrow \pi\right) .
$$

Рассматривая арифметическую реализацию, которая сопоставляет переменной $p$ предложение $\pi$, а $p_{i}$ - предложение $\varphi_{i}$ для всех $i$, по лемме 2.27 заключаем, что

$$
T \vdash \bigwedge_{i=1}^{m}\left(\square_{T} \psi_{i} \rightarrow \psi_{i}\right) \rightarrow \pi,
$$

где $\psi_{i}$ означает арифметическую интерпретацию модальной формулы $Q_{i}$. Заметим теперь, что если $\pi \in \Pi_{n}$ для $n>1$, то $\psi_{i} \in \Pi_{n}$ для всех $i$. Значит,

$$
T+\operatorname{Rfn}_{\Pi_{n}}(T) \vdash \pi .
$$

Аналогично, $\Sigma_{n}$-следствия $\operatorname{Rfn}(T)$ содержатся в $\operatorname{Rfn}_{\Sigma_{n}}(T)$, и $\mathscr{B}\left(\Sigma_{1}\right)$-следствия $\operatorname{Rfn}(T)$ содержатся в теории $\operatorname{Rfn}_{\mathscr{B}\left(\Sigma_{1}\right)}(T)$, которая эквивалентна $\operatorname{Rfn}_{\Sigma_{1}}(T)$ по лемме 2.26.

Следуюшее утверждение показывает, что доказанная вьше теорема оптимальна в смысле арифметической сложности.

ЛЕмма 2.36. При условии непротиворечивости $T_{\omega}$, для $n>1$ найдется $\Sigma_{n}$ (соответственно $\left.\Pi_{n}-\right)$ предложение, доказуемое в $T+\operatorname{Rfn}(T)$, но не доказуемое в $T+\operatorname{Rfn}_{\Pi_{n}}(T)$ (соответственно $\left.T+\operatorname{Rfn}_{\Sigma_{n}}(T)\right)$.

ДокАЗАТЕЛЬСтво. По предложению 2.25 в качестве такой формулы можно взять соответствуюший частный случай схемы $\operatorname{Rfn}_{\Sigma_{n}}(T)$ (соответственно $\operatorname{Rfn}_{\Pi_{n}}(T)$ ).

ЗАмЕчАниЕ 2.37. В настоящее время остается, по-видимому, открытым вопрос об оптимальности утверждения (iii) предыдущей теоремы. В частности, не известно, является ли схема $\operatorname{Rfn}(T) \Pi_{2}$-консервативной над $T+\operatorname{Rfn}_{\Sigma_{1}}(T)$.

В заключение отметим следуюшее усиление следствия 2.19 .

СЛЕДСТВИЕ 2.38. $T+\operatorname{Rfn}(T) \nvdash \operatorname{RFN}(T)$, если и только если теория $T_{\omega}$ непротиворечива.

ДоКАЗАТЕЛЬСТво. В силу следствия 2.35 если $T_{\omega}$ противоречива, то и $T+\operatorname{Rfn}(T)$ - противоречивая теория, т.е. $T+\operatorname{Rfn}(T) \vdash \operatorname{RFN}(T)$. C другой стороны, если $T+$ $\operatorname{Rfn}(T) \vdash \operatorname{RFN}(T)$, то в силу конечной аксиоматизируемости $\operatorname{RFN}_{\Sigma_{1}}(T)$ найдутся некоторые $n$ частных случаев схемы $\operatorname{Rfn}(T)$, из которых выводима $\operatorname{RFN}_{\Sigma_{1}}(T)$. Однако $T+\operatorname{RFN}_{\Sigma_{1}}(T)$ содержит $T_{\omega}$, а значит, и $\operatorname{Con}\left(T_{n}\right)$. Таким образом, в силу предложения $2.30 T_{n}$ и $T_{\omega}$ противоречивы. 


\section{§ 3. Доказуемо тотальные вычислимые функции и фрагменты РА}

Понятие доказуемо тотальной вычислимой функции ${ }^{2}$ было введено Г. Крайзелем в 50-е годы. Это понятие в настоящее время играет исключительно важную роль в теории доказательств в связи как с теоретическими вопросами ординального анализа и извлечения конструктивных оценок из теорем сушествования, так и с более прикладными вопросами, такими как извлечение программ из формальных доказательств. Оно также тесно связано с построением комбинаторных независимых утверждений для арифметики Пеано и ее расширений.

ОПРЕДЕЛЕНИЕ 1. ФУнкци $f: \mathbb{N}^{k} \rightarrow \mathbb{N}$ назьвается доказуемо тотальной вычислимой в теории $T$, если для некоторой $\Sigma_{1}$-формулы $\varphi(\vec{x}, y)$

(i) $f(\vec{x})=y \Longleftrightarrow \mathbb{N} \vDash \varphi(\vec{x}, y)$;

(ii) $T \vdash \forall \vec{x} \exists$ ! $y \varphi(\vec{x}, y)$.

Класс всех доказуемо тотальных вычислимых функций теории $T$ обозначается $\mathscr{F}(T)$.

Заметим, что условие (i) эквивалентно вычислимости функщии $f$. Для теорий $T$, содержащих ЕА, класс $\mathscr{F}(T)$ содержит все элементарные функции $\mathscr{E}$ и замкнут относительно композиции. Вообще говоря, этот класс не является замкнутьм относительно ограниченной минимизации (см. [17]). Отметим также, что $\mathscr{F}(T)$ зависит лишь от $\Pi_{2}$-следствий теории $T$, таким образом, если $T \subseteq_{\Pi_{2}} U$, то $\mathscr{F}(T) \subseteq \mathscr{F}(U)$.

Для многих естественных теорий $T$ было получено описание классов $\mathscr{F}(T)$ в теоретико-рекурсивных терминах. Например, согласно известной теореме, установленной Ч. Парсонсом [65] и независимо Г. Е. Минщем [57], $\mathscr{F}\left(I \Sigma_{1}\right)$ совпадает с классом примитивно рекурсивных функций. Аналогично, из результатов В. Аккермана [1] и Г. Крайзеля [47] следует, что $\mathscr{F}(\mathrm{PA})$ совпадает с классом так называемых $<\varepsilon_{0}$-рекурсивных функций. Характеризация классов $\mathscr{F}(T)$ для сильных теорий $T$ в терминах трансфинитных иерархий рекурсивных функций является одной из основных задач ординального анализа. Обзор [68] содержит обильную информацию, касающуюся этой важной области теории доказательств.

3.1. Фрагменты РА. Основные фрагменты арифметики определяются теми или иньми ограничениями схемы индукции, аксиоматизирующей РА. Как правило, рассматривают следуюшие виды ограничений. Во-первых, накладьвается ограничение на кванторную сложность индукционных формул. Во-вторых, запрещается использование параметров в схеме индукции. В-третьих, иногда индукция применяется в форме правила вьвода, а не как схема аксиом. Правило индукции дает эквивалентную аксиоматизацию РА. Однако схема и правило индукции для формул ограниченной кванторной сложности приводят к различным иерархиям фрагментов арифметики. Систематическое изучение этих иерархий, а также других естественных фрагментов РА, начатое Ч. Парсонсом в начале 70-х годов, к настоящему времени представляет собой активно развиваюшуюся область исследований (обширную библиографию см. в [39]).

Рис. 2 показьвает соотношения между фрагментами РА, определяемыми ограничениями схемы индукции над ЕА. При этом используются следуюшие обозначения.

\footnotetext{
${ }^{2}$ Иногда используется более ранний, но не совсем корректный термин для этого понятия доказуемо рекурсивная функиия.
} 
Теория $I \Sigma_{n}$ определяется над ЕА схемой индукции для $\Sigma_{n}$-формул с параметрами. $I \Sigma_{n}$ эквивалентна $I \Pi_{n}$ (см. ниже лемму 4.1). Теории $I \Sigma_{n}^{-}$и $I \Pi_{n}^{-}$аксиоматизируются соответствуюшими схемами индукции без параметров. $I \Sigma_{n}^{R}$ есть замькание ЕА относительно правила индукции:

$$
\text { IR: } \frac{\varphi(0), \forall x(\varphi(x) \rightarrow \varphi(x+1))}{\forall x \varphi(x)},
$$

где $\varphi \in \Sigma_{n}$. Как показал Ч. Парсонс [66], теория $I \Sigma_{n}^{R}$ эквивалентна $I \Pi_{n+1}^{R}$ (см. ниже следствие 4.14). В [18] показано, что беспараметрические правила индукции эквивалентны правилам индукции с параметрами (см. лемму 4.6). $I \Sigma_{1}^{R}$ эквивалентна обсуждаемой ниже примитивно рекурсивной арифметике PRA.

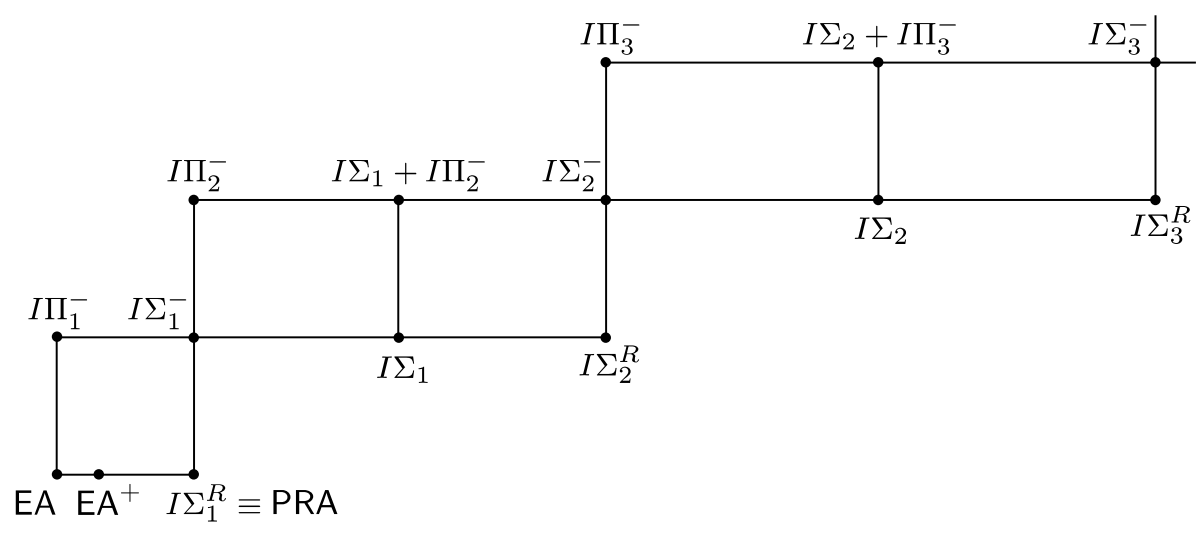

Рис. 2. Фрагменты РА, задаваемые ограниченной индукцией

Среди фрагментов РА между ЕА и $I \Sigma_{1}^{R}$ для нас будет представлять наибольший интерес расширение ЕА аксиомой, утверждающей, что значение функции $2_{n}^{x}$ определено при любых значениях аргументов $n, x$. График функции $2_{n}^{x}$ нетрудно определить элементарной формулой, поскольку

$$
2_{n}^{x}=y \leftrightarrow \exists s \in S e q\left((s)_{0}=x \wedge \forall i<n(s)_{i+1}=\exp \left((s)_{i}\right) \wedge(s)_{n}=y\right),
$$

и квантор существования по $s$ может быть ограничен некоторой элементарной функцией $y$, зависящей от выбранного кодирования последовательностей. Мы обозначаем эту теорию $\mathrm{EA}^{+}$(эквивалентная теория обозначается $I \Delta_{0}+$ Suрехр в [39]). Отметим, что в $\mathrm{EA}^{+}$можно ввести новый символ для функции $2_{n}^{x}$, причем в соответствуюшем дефинищиальном расширении доказуема схема индукции для ограниченных формул расширенного языка (см. [39; с. 271]).

Теория $\mathrm{EA}^{+}$достаточно сильна, чтобы доказать теорему об устранении сечения в логике предикатов и некоторые ее важные следствия такие, как теорема Эрбрана (см. ниже п. 4.1 и [39], [94]). Фактически, по модулю ЕА теория ЕА ${ }^{+}$эквивалентна теореме об устранении сечения. Это следует из хорошо известных верхних и нижних оценок удлинения выводов после устранения правила сечения, полученных Р. Стэтменом [90] и В. П. Оревковым [63]. 
С точки зрения теории доказательств фрагменты РА представляют интерес, поскольку их свойства часто резко отличаются от свойств самой теории РА. Для каждого конкретного фрагмента обычно обсуждаются следуюшие стандартные вопросы:

- конечная аксиоматизируемость теории;

- оптимальная арифметическая сложность ее аксиоматизации;

- класс доказуемо тотальных вычислимых функций данной теории;

- насколько сильные схемы рефлексии выводимы в данной теории над более слабым фрагментом;

- является ли данная теория консервативной над более слабым фрагментом для предложений определенного класса кванторной сложности.

В $\S \S 4,5$ мы установим основные свойства этого рода для фрагментов РА, определяемых ограничениями схемы индукции. Здесь мы рассмотрим более подробно обшие свойства доказуемо тотальных вычислимых функций и их связь со схемами рефлексии.

3.2. Доказуемо тотальные вычислимые функции и 1-непротиворечивость. Предложение 3.1 ниже более или менее непосредственно вытекает из известной теоремы Эрбрана. Напомним, что в простейшем случае теорема Эрбрана утверждает, что для всякой теории $T$ первого порядка, аксиоматизируемой множеством чисто универсальных (или бескванторных) формул, если $T \vdash \exists x \varphi(a, x)$, где $\varphi(a, x)$ бескванторна, то для некоторой последовательности термов $t_{1}(a), \ldots, t_{k}(a)$

$$
T \vdash \varphi\left(a, t_{1}(a)\right) \vee \cdots \vee \varphi\left(a, t_{k}(a)\right)
$$

Эта теорема доказьвается ограниченной индукцией по вьводу без сечения и потому формализуема в $\mathrm{EA}^{+}$(см. [78]). Имеется также простое (но менее конструктивное) теоретико-модельное доказательство, опирающееся на теорему о компактности.

Для данной функции $f$ обозначим через $\mathbf{C}(f)$ замыкание $\mathscr{E} \cup\{f\}$ относительно композиции.

ПРЕДЛОЖЕНИЕ 3.1. Пусть $\pi=\forall x \exists y \varphi(x, y)$ - истинное $\Pi_{2}$-предложсение, где формула ч әлементарна. Тогда $\mathscr{F}(\mathrm{EA}+\pi)=\mathbf{C}(f)$, где $f(x)=\mu y . \varphi(x, y)$.

ДокАЗАТЕЛЬСТво. Обозначим через $T$ теорию ЕА $+\pi$. График $f$ можно задать формулой $\varphi(x, y) \wedge \forall z<y \neg \varphi(x, z)$, отсюда $\mathbf{C}(f) \subseteq \mathscr{F}(T)$.

Для доказательства противоположного включения рассмотрим чисто универсальную аксиоматизацию ЕА в язьке с символами для всех элементарных функций и добавим в язык новый функциональный символ $f$ вместе с аксиомой $\forall x \varphi(x, f(x))$. Используя подходящие элементарные термы, мы можем избавиться от всех ограниченных кванторов в $\varphi$. Поэтому получившаяся теория является консервативньм расширением $T$ и имеет чисто универсальную аксиоматизацию.

Допустим теперь, что $T \vdash \forall x \exists ! y \exists z \psi_{0}(x, y, z)$, где формула $\psi_{0}$ элементарна (а в нашей формулировке эквивалентна бескванторной). По теореме Эрбрана мы получаем термы $t_{1}, \ldots, t_{k}, u_{1}, \ldots, u_{k}$ расширенного языка такие, что

$$
T \vdash \psi_{0}\left(a, t_{1}(a), u_{1}(a)\right) \vee \cdots \vee \psi_{0}\left(a, t_{k}(a), u_{k}(a)\right)
$$


Очевидно, термы $t_{i}$ и $u_{i}$ представляют функции в $\mathbf{C}(f)$. Теперь мы полагаем

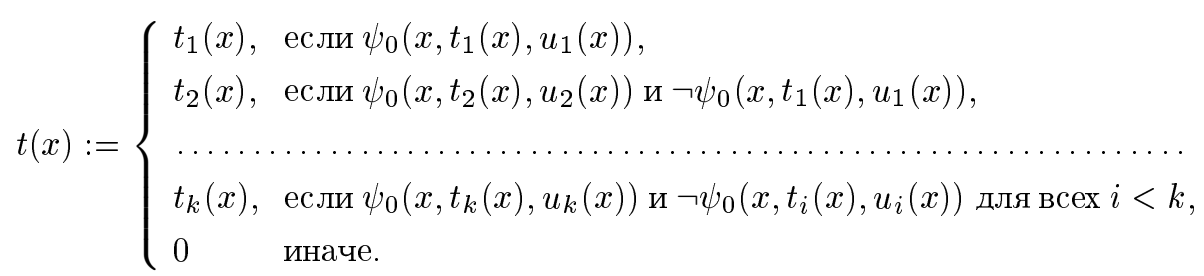

Функция $u(x)$ определяется аналогично, с термами $u_{i}$ вместо $t_{i}$. Поскольку функция

$$
\operatorname{Cond}(x, y, z):= \begin{cases}x, & \text { если } z=0 \\ y, & \text { если } z \neq 0\end{cases}
$$

элементарна, класс $\mathbf{C}(f)$ замкнут относительно определений разбором случаев, и, таким образом, $t(x)$ и $u(x)$ определяются некоторыми $\mathbf{C}(f)$-термами. Для этих термов мы, очевидно, имеем $T \vdash \psi_{0}(a, t(a), u(a))$. Отсюда следует, что

$$
T \vdash \forall x \exists z \psi_{0}(x, t(x), z),
$$

и в силу функциональности $\psi(x, y)=\exists z \psi_{0}(x, y, z)$

$$
T \vdash \forall x, y(t(x)=y \leftrightarrow \psi(x, y)) .
$$

Поскольку теория $T$ корректна, $\psi$ представляет график $t(x)$ в стандартной модели.

СлЕДСТВИЕ 3.2 .

(i) $\mathscr{F}(\mathrm{EA})=\mathscr{E}$.

(ii) $\mathscr{F}\left(\mathrm{EA}^{+}\right)=\mathbf{C}($ supexp$)$, əдe $\operatorname{supexp}(n, x)=2_{n}^{x}$.

Зафиксируем теперь какую-либо естественную нумерацию вычислимых функций (от одной переменной) и связанную с ней $\Sigma_{1}$-формулу $\varphi_{e}(x) \simeq y$, выражающую утверждение, что вычислимая функция с индексом $e$ на входе $x$ выдает ответ $y$. В качестве индексов вычислимых функций $f$ обычно рассматривают коды машин Тьюринга, вычисляюших $f$. Для нас в качестве индексов более удобно рассматривать гёделевы номера $\Sigma_{1}$-формул, определяюших график $f$ в стандартной модели арифметики. Обе эти нумерации эквивалентны в том смысле, что существует взаимно однозначное и (доказуемо) элементарное соответствие между индексами обоих типов. В этом случае формула $\varphi_{e}(x) \simeq y$ выражается через определение истинности для $\Sigma_{1}$-формул как $\operatorname{True}_{\Sigma_{1}}(\operatorname{sub}(e, x, y))$, где элементарньй терм $\operatorname{sub}(e, x, y)$ вычисляет $\ulcorner\sigma(\bar{x}, \bar{y})\urcorner$ по данным $x, y, e$, если $\ulcorner\sigma\urcorner=e$.

Так же, как и для вычислимых функций, мы можем ввести естественную нумерацию доказуемо тотальных вычислимых функций в $T$. 
ОПреДЕЛЕниЕ 2. Пусть $T$ - элементарно аксиоматизированная теория. Называем число е $T$-индексом функции $f$, если $e=\left\langle e_{1}, e_{2}\right\rangle$, где

$-e_{1}$ есть индекс $f$;

- $e_{2}$ кодирует $T$-вьвод формулы $\forall x \exists y \varphi_{\bar{e}_{1}}(x) \simeq y$.

С этой нумерацией $\mathscr{F}(T)$ естественно связывается универсальная функция $\psi^{T}$ :

$$
\psi_{e}^{T}(x):= \begin{cases}\varphi_{e_{1}}(x), & \text { если } e=\left\langle e_{1}, e_{2}\right\rangle \text { есть } T \text {-индекс; } \\ 0 & \text { иначе. }\end{cases}
$$

Обычное диагональное рассуждение показьвает, что $\psi^{T}$, как функция аргументов $e$ и $x$, не принадлежит классу $\mathscr{F}(T)$. Таким образом, утверждение о ее тотальности истинно, но не доказуемо в $T$. Следующая лемма устанавливает эквивалентность этого утверждения и схемы $\Pi_{2}$-рефлексии.

ЛЕмма 3.3. ЕА $\vdash \forall, x \exists y \quad \psi_{e}^{T}(x) \simeq y \leftrightarrow 1$-Con $(T)$.

ДокАЗАТЕльство. Тотальность $\psi^{T}$ выражается формулой

$$
\forall e_{1}, e_{2}, x\left(\operatorname{Prf}_{T}\left(e_{2},\left\ulcorner\forall x \exists y \varphi_{\dot{e}_{1}}(x) \simeq y\right\urcorner\right) \rightarrow \exists y \varphi_{e_{1}}(x) \simeq y\right)
$$

и с точностью до логической эквивалентности представляет собой частный случай схемы $\operatorname{RFN}_{\Pi_{2}}(T)$. Любое $\Pi_{2}$-предложение эквивалентно формуле $\forall x \exists y \varphi_{\bar{e}_{1}}(x) \simeq y$ для подходящего индекса $e_{1}$, таким образом, $(3.1)$ влечет $1-\operatorname{Con}(T)$.

Утверждение о тотальности функции $f$ с определимым графиком будем обозначать $f \downarrow$. При этом подразумевается фиксированной арифметическая формула, задающая график $f$. Как мы видели ранее, всякое истинное $\Pi_{2}$-предложение можно рассматривать как утверждение о тотальности некоторой функции с элементарньм графиком.

Рассмотрим теперь теорию $T$ вида $\mathrm{EA}+f \downarrow$, где $f$ - такая функция. Предложение 3.1 доставляет нумерацию функций из $\mathscr{F}(T)=\mathbf{C}(f)$, не использующую понятие вьвода. Термы, построенные из термов для элементарных функций и символа $f$, имеют естественную гёделеву нумерацию. Мы можем рассматривать эти гёделевы номера как индексы доказуемо тотальных вычислимых функций теории $T$. С данной нумерацией естественно связьвается другая универсальная функция $\theta_{e}^{f}(x)$, вьгисляющая значение терма с номером $e$ на аргументе $x$. Отметим, что обе эти нумерации являются доказуемо эквивалентными в теории $\mathrm{EA}^{+}$, поскольку теорема Эрбрана формализуема в $\mathrm{EA}^{+}$и позволяет явно извлечь терм из доказательства тотальности вычислимой функции.

Пусть функция $f$ имеет элементарньй график, не убьвает и $f(x) \geqslant 2^{x}$. Итерация $f^{(k)}(x)$ функции $f$ определяется как

$$
f^{(0)}(x)=x, \quad f^{(k+1)}(x)=f\left(f^{(k)}(x)\right) .
$$

ЛЕмма 3.4. $\mathbf{C}\left(\theta^{f}\right)=\mathbf{C}\left(f^{*}\right)$, əде $f^{*}(x)=f^{(x)}(x)$. 
ДокАЗАТЕЛЬСТВо. Для каждого фиксированного $k$ функция $f^{(k)} \in \mathbf{C}(f)$ и индекс $f^{(k)}$ является элементарной функцией $k$, скажем, $i(k)$. Отсюда

$$
f^{*}(x)=\theta_{i(x)}^{f}(x) .
$$

Для доказательства противоположной импликации мы опираемся на монотонность $f$. При сделанных предположениях любая функци $g \in \mathbf{C}(f)$ мажорируется некоторой итерацией $f$ :

$$
\forall x\left(g(x) \leqslant f^{(k)}(x)\right) .
$$

При этом число $k$ является элементарной функцией индекса $e=\ulcorner g\urcorner$, скажем, $j(e)$. Мы можем считать $j$ монотонной функцией $e$.

Вычисление значения $\theta_{e}^{f}(x)$ сводится к последовательному вычислению значений подтермов терма $g$. Назовем протоколом вычисления $g(x)$ дерево, листья которого помечены аргументом $x$, корень помечен парой вида $\langle e, y\rangle$, а внутренние вершины помечены парами $\left\langle i, y_{i}\right\rangle$, где $i$ - индекс подтерма $g$, а $y_{i}$ - значение подтерма $i$ на аргументе $x$. При этом $y_{i}$ получается применением элементарных функций или функции $f$ из соответствуюших значений для потомков данной вершины.

Количество всех подтермов и их индексы ограничены индексом $g$. Поэтому все промежуточные значения подтермов мажорируются величиной $f^{(j(e))}(x) \leqslant$ $f^{*}(\max (j(e), x))$. Значит, код всего протокола вычисления $\theta_{e}^{f}(x)$ ограничен некоторой функцией $b(e, x) \in \mathbf{C}\left(f^{*}\right)$. Отсюда получаем представление $\theta^{f}$ в виде:

$$
\theta_{e}^{f}(x)=U(\mu z \leqslant b(e, x) . T(e, x, z)),
$$

где $T(e, x, z)$ - элементарньй предикат, выражающий утверждение " $z$ есть протокол вычисления функции с индексом $e$ на входе $x$ ", а $U(z)$ - элементарная функция, восстанавливаюшая по протоколу значение функции.

Следующая лемма получается формализацией предыдушего утверждения в ЕА.

ЛЕмма 3.5. ЕА $\vdash f^{*} \downarrow \leftrightarrow\langle 1\rangle_{\text {EA }} f \downarrow$.

ДоКАЗАТЕЛЬСТВО. Пусть $T:=\mathrm{EA}+f \downarrow$. По лемме $3.3\langle 1\rangle_{\text {ЕА }} f \downarrow$ эквивалентно в ЕА формуле $\psi^{T} \downarrow$. Формула $\psi^{T} \downarrow$, в частности, влечет $\operatorname{supexp} \downarrow$ и поэтому $\theta^{f} \downarrow$. Обратное также верно, поэтому достаточно показать, что $f^{*} \downarrow$ эквивалентно тотальности $\theta^{f}$.

Очевидно, если $\theta^{f}$ тотальна, то $f^{*}$ также тотальна в силу (3.2).

Для доказательства обратной импликации в силу (3.3) достаточно установить $\forall e, x \exists z \quad T(e, x, z)$ в предположении тотальности $f^{*}$. Рассуждая в $\mathrm{EA}$, рассмотрим произвольные $e, x$. Поскольку $f^{*}$ тотальна, сушествует число $a=b(e, x)$, ограничивающее код протокола из (3.3). Тогда индукцией по $e_{1}$ мы можем доказать

$$
\forall e_{1} \leqslant s e \exists z_{1} \leqslant a T\left(e_{1}, x, z_{1}\right),
$$

где $e_{1} \leqslant_{s} e$ означает, что $e_{1}$ есть подтерм $e$. Как нетрудно видеть, формула индукции ограничена ( $a, e$ и $x$ являются параметрами), поэтому рассуждение формализуемо в EA.

Для функции $f(x)=\exp (x)$ мы получаем следуюшее утверждение. 
СлЕДСТвИЕ 3.6. $\mathrm{EA}^{+} \equiv \mathrm{EA}+\mathrm{RFN}_{\Pi_{2}}(\mathrm{EA})$.

В качестве обобщения этого примера рассмотрим так называемую иерархию Гжегорчика, задаваемую последовательностью функций

$$
F_{0}(x):=2^{x} ; \quad F_{n+1}(x):=F_{n}^{(x)}(x) .
$$

Функции $F_{n}$ примитивно рекурсивны и имеют элементарные графики. Обозначим $\mathscr{E}^{n}:=\mathbf{C}\left(F_{n}\right)$. Как хорошо известно, последовательность классов $\mathscr{E}^{0} \subset \mathscr{E}^{1} \subset \mathscr{E}^{2} \subset \ldots$ исчерпывает все примитивно рекурсивные функции (см. [74]). Расширение ЕА аксиомами $F_{n} \downarrow$ для всех $n \geqslant 1$ представляет собой аксиоматизацию примитивно рекурсивной арифметики PRA.

ПРЕДЛОЖЕНИЕ 3.7.

(i) $\mathrm{EA}_{\omega}^{1} \equiv I \Sigma_{1}^{R} \equiv \mathrm{PRA}$.

(ii) $\mathscr{F}\left(\mathrm{EA}_{\omega}^{1}\right)$ совпадает с классом примитивно рекурсивных функиий.

ДокАЗАТЕЛЬСТво. (i) Тотальность всех $F_{n}$ легко установить с помощью правила $\Sigma_{1}$-индукции. Включение $\mathrm{EA}_{\omega}^{1} \subseteq \mathrm{PRA}$ получается итерацией леммы 3.5. Докажем, что $\mathrm{EA}_{\omega}^{1}$ замкнута относительно правила $\Sigma_{1}$-индукции.

Для данной $\varphi \in \Sigma_{1}$ обозначим через $\psi$ универсальное замыкание

$$
\varphi(0) \wedge \forall x(\varphi(x) \rightarrow \varphi(x+1)) .
$$

Если $\mathrm{EA}_{k}^{1} \vdash \psi$, то

$$
\mathrm{EA}_{k+1}^{1} \vdash \mathrm{RFN}_{\Pi_{2}}(\mathrm{EA}+\psi) .
$$

$\mathrm{C}$ другой стороны, нетрудно видеть, что для каждого конкретного $n \in \mathbb{N}$ EA $+\psi \vdash$ $\varphi(\bar{n})$. Этот факт формализуем в ЕА ограниченной индукцией по $n$, поэтому

$$
\mathrm{EA} \vdash \forall x \square_{\mathrm{EA}+\psi} \varphi(\dot{x}) .
$$

Отсюда с помошюю П казать.

(ii) Любая функция $\mathscr{F}(\mathrm{PRA})$ примитивно рекурсивна, поскольку таковы все $F_{n}$. Тотальность любой примитивно рекурсивной функции естественным образом устанавливается в $I \Sigma_{1}^{R}$.

\section{§4. Индукция и рефлексия}

В этом и последуюших параграфах мы приводим основные результаты, касаюшиеся взаимоотношений между схемами рефлексии и различными формами индукции в арифметике. Первый и наиболее известньй результат этого типа был получен Г. Крайзелем и А. Леви [50], которые доказали, что РА эквивалентна полной равномерной схеме рефлексии над примитивно рекурсивной арифметикой. Впоследствии эта теорема была усилена Д. Лейвантом [51], который установил точную связь между иерархией теорий $I \Sigma_{n}$ и иерархией схем частичной равномерной рефлексии. Ниже мы также даем точные характеризации правил индукции и схем беспараметрической индукции в терминах схем рефлексии, полученные в работах [14], [16]. На основе 
результатов $\S 2$ эти соотношения позволяют получить ряд интересных следствий о строении этих теорий и их классах доказуемо тотальных вычислимых функций.

4.1. Исчисление Тейта. Мы опираемся на стандартную технику устранения сечения в секвенщиальном исчислении Тейта, изложенную, например, в статье Швихтенберга [78]. Формульи в исчислении Тейта строятся, как и в логике первого порядка, из атомарных формул и их отрищаний с помощью связок $\wedge, \vee$ и кванторов $\forall, \exists$. При этом также удобно считать, что алфавиты связанных и свободных переменных не пересекаются. Операция отрицания формулы определена по правилам де Моргана. Секвениии представляют собой конечные множества формул, обозначаемые $\Gamma, \Delta, \ldots$ и понимаемые как дизъюнкции входящих в них формул. $\Gamma \cup\{\varphi\}$ записывается как $\Gamma, \varphi$.

Исчисление Тейта задается аксиомами вида $\Gamma, \varphi, \neg \varphi$, где $\varphi$ - атомарная формула, и следуюшими правилами вьвода:

$$
\begin{gathered}
\frac{\Gamma, \varphi \Gamma, \psi}{\Gamma, \varphi \wedge \psi}(\wedge), \quad \frac{\Gamma, \varphi}{\Gamma, \varphi \vee \psi}\left(\vee_{0}\right), \quad \frac{\Gamma, \psi}{\Gamma, \varphi \vee \psi}\left(\vee_{1}\right), \\
\frac{\Gamma, \varphi(a)}{\Gamma, \forall x \varphi(x)}(\forall), \quad \text { где } a \text { не входит свободно в } \Gamma, \\
\frac{\Gamma, \varphi(t)}{\Gamma, \exists x \varphi(x)}(\exists), \quad \frac{\Gamma, \varphi \quad \Gamma, \neg \varphi}{\Gamma}(C u t) .
\end{gathered}
$$

Нетрудно показать, что секвенция Г является вьводимой в исчислении Тейта тогда и только тогда, когда формула $\bigvee$ Г вьводима в чистом исчислении предикатов РС. Теорема об устранении сечения (см. [78]) утверждает, что существует эффективная процедура, переводящая любой вьвод секвенщии Г в исчислении Тейта в некоторьй вьвод $\Gamma$, не содержаший применений правила ( $C u t)$. Вьводы без сечений обладают тем свойством, что всякая формула, входящая в такой вывод, является подформулой некоторой формулы заключительной секвенции. (При этом свойство быть подформулой понимается с точностью до подстановки термов вместо свободных переменных.) В частности, вывод $\Pi_{n}$-формулы без сечений состоит целиком из $\Pi_{n}$-формул.

Из статьи [78] можно извлечь грубую верхнюю оценку на размер вывода без сечения порядка $2_{c n}^{x}$, где $n$ - ранг сечений, $x$ - размер исходного вьвода, а $c$ - некоторая константа. Это позволяет непосредственно формализовать в ЕА ${ }^{+}$обычное индуктивное доказательство теоремы об устранении сечени (см. также [39; с. 376]).

Для язька исчисления Тейта технически удобно распространить синтаксическое понятие $\Pi_{n}$-формулы на все формулы, получаюшиеся из $\Sigma_{n-1}-$ и $\Pi_{n-1}$-формул с помошью связок $\wedge, \vee$ и квантора $\forall$. В этом и следующем параграфах мы будем работать в исчислении Тейта для арифметического языка без символа экспоненты. Определение истинности для $\Delta_{0}$-формул, выражаемое элементарной формулой в $\mathrm{EA,} \mathrm{построено}$ в [39; с. 364]. На основе этого, как в [39; с. 58-59], строятся определения истинности для классов $\Sigma_{n}$ и $\Pi_{n}$ арифметической иерархии при $n \geqslant 1$.

Помимо утверждения 2.6 нам будут нужны некоторые дополнительные свойства определения истинности для $\Pi_{n}$-формул, называемые условиями Тарского. Условия Тарского формально выражают тот факт, что определение истинности $\operatorname{True}_{n}(x)$ корректно определено на атомарных формулах и доказуемо в ЕА коммутирует с булевыми 
связками и кванторами на множестве $\Pi_{n}$-формул. Другими словами, в $\mathrm{EA}$ доказуемо: для любых формул $\varphi, \psi, \theta, \alpha, \gamma$ таких, что $\varphi, \neg \varphi, \psi, \theta, \forall x \gamma(x), \exists x \alpha(x) \in S t \cap \Pi_{n}$,

$$
\begin{aligned}
\operatorname{True}_{\Pi_{n}}(\ulcorner\neg \varphi\urcorner) & \leftrightarrow \neg \operatorname{True}_{\Pi_{n}}(\ulcorner\varphi\urcorner), \\
\operatorname{True}_{\Pi_{n}}(\ulcorner\theta \wedge \psi\urcorner) & \leftrightarrow \operatorname{True}_{\Pi_{n}}(\ulcorner\theta\urcorner) \wedge \operatorname{True}_{\Pi_{n}}(\ulcorner\psi\urcorner), \\
\operatorname{True}_{\Pi_{n}}(\ulcorner\theta \vee \psi\urcorner) & \leftrightarrow \operatorname{True}_{\Pi_{n}}(\ulcorner\theta\urcorner) \vee \operatorname{True}_{\Pi_{n}}(\ulcorner\psi\urcorner), \\
\operatorname{True}_{\Pi_{n}}(\ulcorner\exists x \alpha(x)\urcorner) & \leftrightarrow \exists x \operatorname{True}_{\Pi_{n}}(\ulcorner\alpha(\dot{x})\urcorner), \\
\text { True }_{\Pi_{n}}(\ulcorner\forall x \gamma(x)\urcorner) & \leftrightarrow \forall x \operatorname{True}_{\Pi_{n}}(\ulcorner\gamma(\dot{x})\urcorner) .
\end{aligned}
$$

Подчеркнем, что вьшеприведенные условия доказуемы в ЕА не только для индивидуальных формул $\varphi, \psi, \ldots$, но и с кванторами обшности по их гёделевым номерам. (В последнем случае обозначение $\ulcorner\alpha(\dot{x})\urcorner$ следует понимать как определимьй терм для элементарной функции, сопоставляюшей номеру формулы $\alpha$ и числу $x$ номер формулы $\alpha(\bar{x})$. Поскольку ниже мы используем условия Тарского лишш в неформальном контексте, мы не будем вводить специальных обозначений для такого рода функций.)

Мы также предполагаем, что формализация операции $\bigvee \Gamma$ на секвенциях $\Gamma$, состоящих из $\Pi_{n}$-формул, доказуемо в ЕА согласована с $\operatorname{True}_{n}(x)$ в том смысле, что истинность $\bigvee$ Г не зависит от упорядочения формул секвенции Г в дизъюнкцию. В частности, в ЕА доказуемо: для любых $\varphi \in \Gamma \subseteq S t \cap \Pi_{n}$

$$
\operatorname{True}_{\Pi_{n}}(\ulcorner\varphi\urcorner) \rightarrow \operatorname{True}_{\Pi_{n}}(\ulcorner\bigvee \Gamma\urcorner)
$$

Считаем, что для пустой секвенщии $\Gamma \bigvee \Gamma:=\perp$ и тем самым доказуемо $\neg \operatorname{True}_{\Pi_{n}}(\ulcorner\bigvee \Gamma\urcorner)$.

В EA мы также имеем элементарную определимую универсальную функцию для термов языка без экспоненты, т.е. функцию eval $(u, x)$, для любого терма $t\left(x_{0}, \ldots, x_{n}\right)$ удовлетворяющую условию ${ }^{3}$

$$
\mathrm{EA} \vdash \operatorname{eval}\left(\ulcorner t\urcorner,\left\langle x_{0}, \ldots, x_{n}\right\rangle\right)=t\left(x_{0}, \ldots, x_{n}\right) .
$$

Обычно функция eval $(u, x)$ используется явно в конструкции определения истинности для означивания атомарных и ограниченных формул [39; с. 363-364]. Вместе с доказуемой тотальностью eval $(u, x)$ это влечет, что определение истинности и универсальная функция согласованы в том смысле, что в ЕА доказуемо: для любьх $\Pi_{n}$-формул $\varphi\left(z_{0}, \ldots, z_{m}\right)$ и термов $t_{0}(\vec{x}), \ldots, t_{m}(\vec{x})$

$$
\begin{aligned}
& \bigwedge_{i=0}^{m} \operatorname{eval}\left(\left\ulcorner t_{i}\right\urcorner,\langle\vec{x}\rangle\right)=y_{i} \\
& \quad \rightarrow\left(\operatorname{True}_{\Pi_{n}}\left(\left\ulcorner\varphi\left(t_{0}(\dot{\vec{x}}), \ldots, t_{m}(\dot{\vec{x}})\right)\right\urcorner\right) \leftrightarrow \operatorname{True}_{\Pi_{n}}\left(\left\ulcorner\varphi\left(\dot{y}_{0}, \ldots, \dot{y}_{m}\right)\right\urcorner\right)\right) .
\end{aligned}
$$

Здесь и ниже $\vec{x}$ есть сокрашение для $x_{0}, \ldots, x_{k} ; \dot{\vec{x}}$ обозначает $\dot{x}_{0}, \ldots, \dot{x}_{k} ; \overline{\vec{x}}$ обозначает $\bar{x}_{0}, \ldots, \bar{x}_{k} ;$ a $\langle\vec{x}\rangle$ есть $\left\langle x_{0}, \ldots, x_{k}\right\rangle$.

\footnotetext{
${ }^{3}$ Подразумеваем, что все переменные арифометического язька упорядочены в фиксированную последовательность $x_{0}, \ldots, x_{n}, \ldots$.
} 
4.2. Схемы индукции с параметрами. Мы начнем с базисной леммы о схемах индукции ограниченной арифметической сложности, доказанной в [66].

ЛЕмма 4.1. $I \Sigma_{n} \equiv I \Pi_{n}$ для любого $n \geqslant 1$.

ДокАЗАтЕльСтво. Рассмотрим любую $\Pi_{n}$-формулу $\varphi(x)$. Чтобы вьвести пример схемы IA для $\varphi(x)$ с помошњю $\Sigma_{n}$-индукции, рассмотрим $\Sigma_{n}$-формулу $\psi(a, x):=$ $\neg \varphi(a-x)$. Тогда $\forall x(\varphi(x) \rightarrow \varphi(x+1))$ влечет $\forall x \quad(\psi(a, x) \rightarrow \psi(a, x+1))$, следовательно, $\psi(a, 0) \rightarrow \psi(a, a)$ по $I \Sigma_{n}$. Но это означает, что $\varphi(0) \rightarrow \varphi(a)$.

Отметим роль параметра $a$ в этом рассуждении: оно не проходит для теории $I \Sigma_{n}^{-}$.

Следуюшее предложение для случая примитивно рекурсивной арифметики PRA вместо ЕА и $n \geqslant 2$ получено Д. Лейвантом [51]. Случай $n=1$ требует ослабления теории PRA до EA. Включение теорий слева направо в этой теореме впервые было отмечено, по-видимому, Х. Оно [62].

TЕОрема 7. $I \Sigma_{n} \equiv \mathrm{EA}+\operatorname{RFN}_{\Sigma_{n+1}}(\mathrm{EA})$ для любого $n \geqslant 1$.

ДокАЗАТЕЛЬство. $(\subseteq)$ Пусть $\sigma(x, y) \in \Sigma_{n}$, где без ограничения общности мы рассматриваем единственньй параметр $y$. Обозначим через $\psi(y)$ формулу

$$
\sigma(0, y) \wedge \forall x(\sigma(x, y) \rightarrow \sigma(x+1, y))
$$

С помошњю очевидной индукции по $k$ получаем

$$
\forall k, m \in \mathbb{N} \text { EA } \vdash \psi(\bar{m}) \rightarrow \sigma(\bar{k}, \bar{m})
$$

Размер соответствующего ЕА-вьвода оценивается элементарной функцией от $k$ и $m$, поэтому внешняя индукция по $k$ формализуема в $\mathrm{EA}$ :

$$
\mathrm{EA} \vdash \forall x, y \quad \square_{\mathrm{EA}}(\psi(\dot{y}) \rightarrow \sigma(\dot{x}, \dot{y}))
$$

Заметим, что $\psi(y) \rightarrow \sigma(x, y)$ логически эквивалентна $\Sigma_{n+1}$-формуле, поэтому с помощью равномерной схемы $\Sigma_{n+1}$-рефлексии вьводим

$$
\mathrm{EA}+\mathrm{RFN}_{\Sigma_{n+1}}(\mathrm{EA}) \vdash \forall x, y(\psi(y) \rightarrow \sigma(x, y)),
$$

что эквивалентно соответствующему примеру схемы индукции.

$(\supseteq)$ Для доказательства RFN $\Sigma_{n+1}(\mathrm{EA})$ в $I \Sigma_{n}$ заметим, что достаточно вьвести в $I \Sigma_{n}$ схему равномерной $\Sigma_{n+1}$-рефлексии $\mathrm{RFN}_{\Sigma_{n+1}}(\mathrm{PC})$ для чистого исчисления предикатов РС в арифметическом языке без экспоненты. Это следует из доказуемой эквивалентности ЕА и $I \Delta_{0}+$ Ехр и того, что $I \Delta_{0}+$ Ехр имеет конечную $\Pi_{2}$-аксиоматизацию (над РС), откуда в силу аналога леммы 2.23

$$
\begin{aligned}
\mathrm{EA} \vdash \operatorname{RFN}_{\Sigma_{n+1}}(\mathrm{EA}) & \leftrightarrow \operatorname{RFN}_{\Sigma_{n+1}}\left(I \Delta_{0}+\operatorname{Exp}\right) \\
& \leftrightarrow \operatorname{RFN}_{\Sigma_{n+1}}(\mathrm{PC}) .
\end{aligned}
$$


Для заданной $\Sigma_{n+1}$-формулы $\sigma(z):=\exists y_{1} \ldots \exists y_{m} \quad \alpha\left(y_{1}, \ldots, y_{m}, z\right)$, где $\alpha \in \Pi_{n}$, и числа $k \in \mathbb{N}$ рассмотрим вывод без сечения секвенщии $\sigma(\bar{k})$, П для некоторого множества $\Pi_{n}$-формул П. По свойству подформульности любая формула, входящая в этот вьвод, или (а) имеет вид

$$
\exists y_{i} \ldots \exists y_{m} \alpha\left(t_{0}, \ldots, t_{i-1}, y_{i}, \ldots, y_{m}, \bar{k}\right)
$$

для некоторого $0 \leqslant i \leqslant m$ и термов $t_{0}, \ldots, t_{i-1}$; или $(\mathrm{b})$ есть $\Pi_{n}$-формула.

Пусть $I_{\sigma}(h, k)$ означает $\Pi_{n}$-формулу, естественньм образом выражающую следующее:

“Для любого р, если р есть код вывода без сечения секвенции вида $\Gamma(\bar{k}), \Pi(\vec{a})$,

где $\Gamma(\bar{k})$ есть множество формул вида (а) выше, П $(\vec{a})$ есть множество

$\Pi_{n}$-формул, и если выссота выввода р меньше $h$, то $\forall \vec{x} \quad \operatorname{True}_{\Pi_{n}}(\ulcorner\bigvee \Pi(\dot{\vec{x}})\urcorner)$ ".

ЛЕмма 4.2. ЕA $\vdash \neg(z) \rightarrow\left(I_{\sigma}(0, z) \wedge \forall h \quad\left(I_{\sigma}(h, z) \rightarrow I_{\sigma}(h+1, z)\right)\right)$.

ДокАЗАТЕльство. Мы докажем лемму, рассуждая неформально внутри ЕА. $I_{\sigma}(0, z)$ имеет место тривиальным образом. Покажем, что $I_{\sigma}(h, z)$ влечет $I_{\sigma}(h+1, z)$. Для этого рассмотрим вывод без сечения высоты $h$ секвенции вида $\Gamma, \Pi(\vec{a})$, где Г и П определены как вьше, и покажем, что дизъюнкция П истинна в смысле True $\Pi_{n}$ при любой подстановке нумералов вместо свободных переменных в П. Мы разбираем несколько случаев, в зависимости от вида последнего применения правила в данном вьводе. У тверждение $I_{\sigma}(h, z)$ мы будем назьвать предположением индукции.

Случай 1. Секвенция $\Gamma, \Pi(\vec{a})$ есть логическа аксиома, т.е. имеет вид $\Delta, \varphi(\vec{a}), \neg \varphi(\vec{a})$ для некоторой атомарной формулы $\varphi$. Поскольку все формулы типа (a) содержат кванторы сушествования и тем самьм не являются атомарными формулами или их отрицаниями, $\varphi$ и $\neg \varphi$ должны входить в П. Условия Тарского влекут, что для любого $\vec{x}$

$$
\operatorname{True}_{\Pi_{n}}(\ulcorner\varphi(\dot{\vec{x}})\urcorner) \vee \operatorname{True}_{\Pi_{n}}(\ulcorner\neg \varphi(\dot{\vec{x}})\urcorner) .
$$

Отсюда мы вьводим $\forall \vec{x} \operatorname{True}_{\Pi_{n}}(\ulcorner\bigvee \Pi(\dot{\vec{x}})\urcorner)$ в силу (4.1).

Случай 2. Секвенция $\Gamma, \Pi(\vec{a})$ получена по правилу введения булевой связки или квантора в формулу из $\Pi(\vec{a})$. Все эти правила рассматриваются аналогично, опираясь на свойство подформульности для вьводов без сечения и условия Тарского для True $_{n}$. Например, правило введения квантора общности имеет вид

$$
\frac{\Gamma, \Pi^{\prime}(\vec{a}), \varphi(\vec{a}, b)}{\Gamma, \Pi^{\prime}(\vec{a}), \forall x \varphi(\vec{a}, x)}
$$

где $b$ не входит свободно в $\Gamma, \Pi^{\prime}(\vec{a})$. Мы должны показать, что для любого набора $\vec{x}$ формула $\bigvee \Pi^{\prime}(\overline{\vec{x}}) \vee \forall y \varphi(\overline{\vec{x}}, y)$ истинна. По предположению индукции, поскольку $b$ не входит свободно в $\Pi^{\prime}$, мы знаем, что для любого $y$ формула $\bigvee \Pi^{\prime}(\overline{\vec{x}}) \vee \varphi(\overline{\vec{x}}, \bar{y})$ истинна. Коммутируя $\operatorname{True}_{\Pi_{n}} \mathrm{c} \vee$, мы получаем, что для любого $y$ или $\bigvee \Pi^{\prime}(\overline{\vec{x}})$, или $\varphi(\overline{\vec{x}}, \bar{y})$ истинна. Поскольку $\operatorname{True}_{\Pi_{n}}\left(\left\ulcorner\bigvee \Pi^{\prime}(\dot{\vec{x}})\right\urcorner\right)$ не зависит от $y$, отсюда вытекает, что или $\bigvee \Pi^{\prime}(\overline{\vec{x}})$ истинна, или для любого $y \quad \varphi(\overline{\vec{x}}, \bar{y})$ истинна. Коммутируя True $\Pi_{n}$ с квантором обшности, а затем с дизъюнкцией в обратном направлении, мы заключаем, что формула $\bigvee \Pi^{\prime}(\overline{\vec{x}}) \vee \forall y \varphi(\overline{\vec{x}}, y)$ истинна, что и требовалось доказать. 
Случай 3. Последнее применение правила вьвода добавляет квантор существования непосредственно перед $\alpha$, т.е. вьвод имеет вид

$$
\frac{\Gamma^{\prime}, \alpha\left(t_{0}(\vec{a}), \ldots, t_{m-1}(\vec{a}), t_{m}(\vec{a}), \bar{z}\right), \Pi(\vec{a})}{\Gamma^{\prime}, \exists y_{m} \alpha\left(t_{0}(\vec{a}), \ldots, t_{m-1}(\vec{a}), y_{m}, \bar{z}\right), \Pi(\vec{a})} .
$$

По предположению индукции или дизъюнкция П $(\overline{\vec{x}})$, или формула

$$
\alpha\left(t_{0}(\overline{\vec{x}}), \ldots, t_{m}(\overline{\vec{x}}), \bar{z}\right)
$$

истинна. Нам нужно опровергнуть вторую возможность, рассужда внутри ЕА.

Заметим, что хотя, вообше говоря, $t_{i}$ являются "нестандартными” термами, $\alpha-$ фиксированна "стандартная" $\Pi_{n+1}$-формула. Поэтому предложение 2.6 может быть применено к $\alpha$ после вычисления значений термов $t_{i}$. Так, по (4.2) и предложению 2.6 мы получаем:

$$
\begin{aligned}
& \operatorname{True}_{\Pi_{n}}\left(\left\ulcorner\alpha\left(t_{0}(\dot{\vec{x}}), \ldots, t_{m}(\dot{\vec{x}}), \dot{z}\right)\right\urcorner\right) \wedge \bigwedge_{i=0}^{m} \operatorname{eval}\left(\left\ulcorner t_{i}\right\urcorner,\langle\vec{x}\rangle\right)=y_{i} \\
& \quad \rightarrow \operatorname{True}_{\Pi_{n}}\left(\left\ulcorner\alpha\left(\dot{y}_{0}, \ldots, \dot{y}_{m}, \dot{z}\right)\right\urcorner\right) \\
& \quad \rightarrow \alpha\left(y_{0}, \ldots, y_{m}, z\right) .
\end{aligned}
$$

Поскольку универсальная функция доказуемо тотальна в ЕА, отсюда вытекает, что формула

$$
\operatorname{True}_{\Pi_{n}}\left(\left\ulcorner\alpha\left(t_{0}(\dot{\vec{x}}), \ldots, t_{m}(\dot{\vec{x}}), \dot{z}\right)\right\urcorner\right)
$$

влечет $\exists y_{0} \ldots \exists y_{m} \quad \alpha\left(y_{0}, \ldots, y_{m}, z\right)$, т.е. противоречит гипотезе $\neg \sigma(z)$. Таким образом, для любых $t_{0}, \ldots, t_{m}$ и $\vec{x}$ формула $\alpha\left(t_{0}(\overline{\vec{x}}), \ldots, t_{m}(\overline{\vec{x}}), \bar{z}\right)$ не может быть истинной, значит, истинна дизъюнкция П $(\overline{\vec{x}})$.

Случай 4. Г, П $(\vec{a})$ получается по правилу, вводяшему любой другой квантор существования в формулу из Г. Тогда наше утверждение непосредственно следует из предположения индукции, поскольку П-часть посылки в этом случае совпадает с соответствующей частью заключения.

Поскольку для $n \geqslant 1 I \Sigma_{n}$ эквивалентна $I \Pi_{n}$ и содержит $\mathrm{EA}^{+}$, из предыдушей леммы непосредственно вытекает, что

$$
I \Sigma_{n} \vdash \neg \sigma(z) \rightarrow \forall h \quad I_{\sigma}(h, z)
$$

Чтобы вьвести RFN $\Sigma_{n+1}(\mathrm{PC})$, мы рассуждаем теперь внутри $I \Sigma_{n}$ для каждой $\Sigma_{n+1^{-}}$ формулы $\sigma(z)$ следуюшим образом.

Допустим $\neg \sigma(z)$ и $\square \mathrm{PC} \sigma(\dot{z})$, тогда секвенция, состоящая из единственной формулы $\sigma(\bar{z})$, доказуема в исчислении Тейта. По (формализованной) теореме об устранении сечения мы получаем вьвод этой секвенции без сечений и по (4.3) заключаем, что имеет место $\operatorname{True}_{\Pi_{n}}(\ulcorner\perp\urcorner)$. Но отсюда следует $\perp$, т.е. противоречие.

Следуюшее утверждение было получено Г. Крайзелем и А. Леви [50]. 
СЛЕДСТВИЕ 4.3. PA $\equiv \mathrm{EA}+\mathrm{RFN}(\mathrm{EA})$.

Как следствие теорем о неограниченности для равномерной рефлексии мы получаем следуюшие результаты, из которых первый получен М. Рабином [71], а второй Д. Лейвантом [51].

СлЕДСТВИЕ 4.4.

(i) PA не содержится ни в одном непротиворечивом расширении ЕА ограниченной арифметической сложности.

(ii) $I \Sigma_{n}$ не содержится ни в одном непротиворечивом расширении ЕА сложности $\Sigma_{n+2}$.

Отметим, что сложность очевидной аксиоматизации $I \Sigma_{n}$ есть $\Pi_{n+2}$.

СлеДСТвиЕ 4.5. Ни $I \Pi_{n+1}^{R}$, ни $I \Pi_{n+1}^{-}$не содержат $I \Sigma_{n}$.

ДокАЗАТЕЛЬСтво. Арифметическая сложность аксиоматизации этих теорий есть $\Pi_{n+1}$ и $\Sigma_{n+2}$ соответственно (все формулы, являюшиеся заключениями примеров правила $\Pi_{n+1}-\mathrm{IR}$, имеют сложность $\left.\Pi_{n+1}\right)$.

4.3. Правила индукции. Для начала мы введем некоторую общую терминологию и обозначения, касаюшиеся правил вьвода в арифметике. Мы назьваем правилом произвольное множество примеров, т.е. выражений вида

$$
\frac{\varphi_{1}, \ldots, \varphi_{n}}{\psi},
$$

где $\varphi_{1}, \ldots, \varphi_{n}$ и $\psi$ - арифметические формулы. Вьводы с использованием правил определяются стандартным образом; $T+R$ означает замыкание теории $T$ относительно правила $R$ и логики первого порядка. $[T, R]$ означает замькание $T$ относительно однократного применения правила $R$, т.е. теорию, аксиоматизированную над $T$ всеми формулами $\psi$ такими, что для некоторых формул $\varphi_{1}, \ldots, \varphi_{n}$, выводимых в $T$, (4.4) есть пример $R$.

Правило $R_{1}$ называется выводимым из $R_{2}$, если для любой теории $T$, содержащей $\mathrm{EA}, T+R_{1} \subseteq T+R_{2}$. Другими словами, это означает, что любой вьвод из посылок с использованием $R_{1}$ можно заменить на эквивалентньй вывод с использованием ЕА и $R_{2}$. Правило $R_{1}$ называется сводимым к $R_{2}$, если для любой теории $T$, содержашей $\mathrm{EA},\left[T, R_{1}\right] \subseteq\left[T, R_{2}\right] . R_{1}$ и $R_{2}$ назьваются конгруәнтныцми, если они взаимно сводимы (обозначается $R_{1} \cong R_{2}$ ). Для теории $U$, содержашей ЕА, мы говорим, что $R_{1}$ и $R_{2}$ конгруэнтны по модулю $U$, если для любого расширения $T$ теории $U$ $\left[T, R_{1}\right] \equiv\left[T, R_{2}\right]$.

Теперь мы рассмотрим более подробно правила индукции для $\Sigma_{n}$ - и $\Pi_{n}$-формул, обозначаемые соответственно $\Sigma_{n}$-IR и $\Pi_{n}$-IR. Вообще говоря, мы допускаем параметры в индукционных формулах этих правил, однако имеет место следуюша полезная лемма.

Лемма 4.6.

(i) $\Pi_{n}$-IR сводится к правилу $\Pi_{n}$-IR без параметров.

(ii) $\Sigma_{n}$-IR сводится $к$ правилу $\Sigma_{n}$-IR без параметров. 
ДокАЗАТЕЛЬСТВО. Применение $\mathrm{IR}$ к формуле $\varphi(x, a)$ с индукционной переменной $x$ и параметром $a$ очевидным образом сводится к применению $\mathrm{IR}$ для формулы $\forall z \varphi(x, z)$, что доказывает первое утверждение леммы.

Допустим теперь, что $\varphi(x, y, a) \in \Pi_{n-1}$ и

$$
\begin{aligned}
& T \vdash \exists y \varphi(0, y, a), \\
& T \vdash \forall x(\exists y \varphi(x, y, a) \rightarrow \exists y \varphi(x+1, y, a)) .
\end{aligned}
$$

Обозначим

$$
\varphi^{\prime}(x, y):=\forall i \leqslant x \varphi\left((i)_{0},(y)_{i},(i)_{1}\right) .
$$

Тогда мы имеем, в силу (4.5) и монотонности кодирования последовательностей, $T \vdash$ $\exists y \varphi^{\prime}(0, y)$. Для доказательства

$$
T \vdash \forall x\left(\exists y \varphi^{\prime}(x, y) \rightarrow \exists y^{\prime} \varphi^{\prime}\left(x+1, y^{\prime}\right)\right)
$$

предположим, что $\forall i \leqslant x \varphi\left((i)_{0},(y)_{i},(i)_{1}\right)$. Если $(x+1)_{0}=0$, то по (4.5) найдется элемент $z$ такой, что $\varphi\left(0, z,(x+1)_{1}\right)$, и в качестве $y^{\prime}$ можно взять последовательность $y *\langle z\rangle$. Если же $(x+1)_{0}>0$, то код пары $p:=\left\langle(x+1)_{0}-1,(x+1)_{1}\right\rangle$ строго меньше $x+1$ и, таким образом (по индуктивному предположению), найдется $z=(y)_{p}$ такой, что $\varphi\left((x+1)_{0}-1, z,(x+1)_{1}\right)$. Из (4.6) следует, что для некоторого $z^{\prime}$ имеет место $\varphi\left((x+1)_{0}, z^{\prime},(x+1)_{1}\right)$. Значит, можно взять в качестве $y^{\prime}$ последовательность $y *\left\langle z^{\prime}\right\rangle$. Таким образом, применение IR для формулы $\exists y \varphi(x, y, a)$ сводится к применению IR для $\Sigma_{n}$-формулы $\exists y \varphi^{\prime}(x, y)$ без параметров.

СЛЕДСТВИЕ 4.7. $I \Sigma_{n}^{R} \subseteq I \Sigma_{n}^{-}, I \Pi_{n}^{R} \subseteq I \Pi_{n}^{-}$.

Теперь мы введем новое правило вывода, связанное со схемой рефлексии. Для данной элементарно аксиоматизированной теории $T$ правилом метарефлексии над $T$ называется правило

$$
\operatorname{RR}^{n}(T): \frac{\varphi(\vec{x})}{\langle n\rangle_{T} \varphi(\overrightarrow{\vec{x}})} .
$$

Это же правило с ограничением $\varphi \in \Pi_{m}$ обозначаетс $\Pi_{m}-\mathrm{RR}^{n}(T)$. В случае $T=\mathrm{EA}$ мы пишем $\Pi_{m}-\mathrm{RR}^{n}$.

Заметим, что $\Pi_{m}-\mathrm{RR}^{n}(T)$ конгруэнтно своему варианту без параметров, поскольку по этому правилу из $\varphi(\vec{x})$ можно вьвести $\langle n\rangle_{T} \forall \vec{x} \varphi(\vec{x})$, откуда $\forall \vec{x}\langle n\rangle_{T} \varphi(\overrightarrow{\vec{x}})$ вытекает в силу условий Лёба.

Следуюшая теорема получена в [11], [14].

TEOPEMA 8 .

(i) $\Pi_{n+1}-\mathrm{IR} \cong \Pi_{n+2^{-}} \mathrm{RR}^{n}$, ecли $n>0$.

(ii) $\Pi_{1}-\mathrm{IR} \cong \Pi_{2}-\mathrm{RR}^{0}$ ( по модулю $\mathrm{EA}^{+}$). 
ДоКАЗАТЕЛЬСТВо. Для сведения $\Pi_{n+1}$-IR к $\Pi_{n+2}-\mathrm{RR}^{n}$ допустим, что теория $T$ ВЬВодит

$$
\varphi(0) \wedge \forall x(\varphi(x) \rightarrow \varphi(S(x))),
$$

где мы можем предполагать, что $\varphi$ не содержит дополнительных параметров. Заметим, что (4.7) логически эквивалентна некоторому $\Pi_{n+2}$-предложению, скажем $\psi$. Для каждого $n \in \mathbb{N}$ мы имеем $\mathrm{EA}+\psi \vdash \varphi(\bar{n})$ и, формализуя этот факт в $\mathrm{EA}$, получаем

$$
\mathrm{EA} \vdash \forall x \square_{\mathrm{EA}+\psi} \varphi(\dot{x}) .
$$

С другой стороны, с помошью метарефлексии из $\psi$ мы выводим

$$
\left[T, \Pi_{n+2^{-}} \mathrm{RR}^{n}\right] \vdash \mathrm{RFN}_{\Pi_{n+1}}(\mathrm{EA}+\psi),
$$

откуда

$$
\left[T, \Pi_{n+2}-\mathrm{RR}^{n}\right] \vdash \forall x \varphi(x),
$$

что и требовалось.

Теперь нам нужно показать, что для $T$, содержащей ЕА (или $\left.\mathrm{EA}^{+}{ }_{\text {при }} n=0\right)$,

$$
\left[T, \Pi_{n+1}-\mathrm{IR}\right] \vdash \mathrm{RFN}_{\Pi_{n+1}}(\mathrm{EA}+\psi)
$$

для любого предложения $\psi \in \Pi_{n+2}$ такого, что $T \vdash \psi$. Без ограничения общности мы можем считать, что $T$ сформулирована в арифметическом языке без экспоненты и $T \equiv I \Delta_{0}+\operatorname{Exp}+\psi$. Используя конечную аксиоматизируемость $I \Delta_{0}+$ Ехр, мы также предполагаем, что единственная нелогическая аксиома $T$ имеет вид $\forall x_{0} \ldots \forall x_{m}$ $\neg \alpha\left(x_{0}, \ldots, x_{m}\right)$, где $\alpha \in \Pi_{n}$. В частности, эта формула аккумулирует все аксиомы равенства и конечную $\Pi_{2}$-аксиоматизацию $I \Delta_{0}+$ Exp.

Для доказательства $\mathrm{RFN}_{\Pi_{n+1}}(T)$ мы рассматриваем в исчислении Тейта произвольные вьводы без сечения секвенций вида

$$
\exists x_{0} \ldots \exists x_{m} \alpha\left(x_{0}, \ldots, x_{m}\right), \Pi,
$$

где П есть множество $\Pi_{n+1}$-формул. В этой ситуации непосредственно применима лемма 4.2: формула $\exists x_{0} \ldots \exists x_{m} \alpha\left(x_{0}, \ldots, x_{m}\right)$, т.е. отрищание аксиомы $T$, играет роль формулы $\sigma$. Параметр z в этом случае отсутствует.

Поскольку $T$ содержит ЕА, по лемме 4.2

$$
T \vdash I_{\sigma}(0) \wedge \forall h\left(I_{\sigma}(h) \rightarrow I_{\sigma}(h+1)\right) .
$$

Поэтому по правилу $\Pi_{n+1}$-IR мы можем вывести

$$
\left[T, \Pi_{n+1}-\mathrm{IR}\right] \vdash \forall h I_{\sigma}(h),
$$

что влечет $\mathrm{RFN}_{\Pi_{n+1}}(T)$ над $\mathrm{EA}^{+}$.

Осталось заметить, что при $n>0$ теория $\left[\mathrm{EA}, \Pi_{n+1^{-}} \mathrm{IR}\right]$ содержит $\mathrm{EA}^{+}$.

Пусть, для фиксированного $n \geqslant 0, T_{k}^{n}$ означает последовательность теорий, основанную на итерации формулы $n$-непротиворечивости над $T$ :

$$
T_{0}^{n}:=T, \quad T_{k+1}^{n}:=T_{k}^{n}+n-\operatorname{Con}\left(T_{k}^{n}\right), \quad T_{\omega}^{n}:=\bigcup_{k \geqslant 0} T_{k}^{n} .
$$

Поскольку формула $n$-Con $(T)$ имеет сложность $\Pi_{n+1}$, теорема 8 может быть применена несколько раз, и мы получаем следуюшее утверждение. 
СлЕДСТВИЕ 4.8. Пусть $T$ - конечная $\Pi_{n+2}$-аксиоматизированная теория, содерәсащая ЕA (или $\mathrm{EA}^{+}$для $\left.n=0\right)$. Тогда

$$
T+\Pi_{n+1}-\mathrm{IR} \equiv T_{\omega}^{n}
$$

Можно отметить также, что $k$-кратное применение правила индукции в точности соответствует $k$ раз итерированной схеме рефлексии над $T$.

СлЕДСТВИЕ 4.9. $I \Pi_{n+1}^{R} \equiv \mathrm{EA}_{\omega}^{n}$ для любого $n>0$.

Интересньй частньй случай следствия 4.8 относится к правилу индукции для $\Pi_{1}$-формул.

СлЕДСТВИЕ 4.10. Для конечных $\Pi_{2}$-аксиоматизированных теорий $T$, содержащит $\mathrm{EA}^{+}$,

$$
T+\Pi_{1}-\mathrm{IR} \equiv T_{\omega} \equiv T+\operatorname{Con}(T)+\operatorname{Con}(T+\operatorname{Con}(T))+\cdots .
$$

С ЛЕДСТВИЕ 4.11. Для $\Pi_{n+1}$-аксиоматизированньх теорий $T$, содержащих $\mathrm{EA}$ $\left(\right.$ или $\mathrm{EA}^{+}$для $\left.n=1\right)$, теория $T+\Pi_{n}$-IR не является конечно аксиоматизируемой, при условии ее непротиворечивости.

СЛЕДСТВИЕ 4.12. Теории $I \Pi_{n}^{R}$ для $n>1$, a maкже теория $\mathrm{EA}^{+}+\Pi_{1}$-IR не являются конечно аксиоматизируемыми.

ЗАмЕчАниЕ 4.13. Характеризация правила $\Pi_{1}$-IR с точностью до конгруэнтности по модулю ЕА может быть получена на основе идей работы А. Уилки и Дж. Париса [94]. В этой ситуации формула непротиворечивости $T$ в смысле доказуемости без

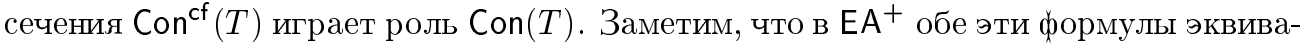
лентны в силу доказуемости теоремы об устранении сечения.

Все фактические применения правила сечения в доказательстве теоремы 8 имеют ограниченный (стандартный) ранг, поэтому их устранимость можно установить уже в EA. C учетом этого все рассуждения проходят без существенных изменений и показывают, что П 1 -IR конгруэнтно правилу

$$
\frac{\varphi}{\operatorname{Con}^{\mathrm{cf}}(\mathrm{EA}+\varphi)}, \quad \varphi \in \Pi_{2} .
$$

В силу результатов [94] отсюда также следует, что $I \Pi_{1}^{R}$ не является конечно аксиоматизируемой теорией.

Доказательство следуюшей теоремы более сложно и мы его опускаем (см. [14]).

\section{TeOpema 9 .}

(i) $\Sigma_{1}-\mathrm{IR} \cong \Pi_{2}-\mathrm{RR}^{1}$.

(ii) $\Sigma_{n}-\mathrm{IR} \cong \Pi_{n+1}-\mathrm{RR}^{n}$ по модулю $I \Sigma_{n-1} \partial$ ภя $>1$.

Поскольку, как нетрудно видеть, [EA, $\Sigma_{n}$-IR] содержит $I \Sigma_{n-1}$, утверждение (ii) влечет, что правила $\Pi_{n+1}-\mathrm{RR}^{n+1}$ и $\Sigma_{n}$-IR взаимно выводимы для всех $n \geqslant 1$. Фактически, $k$ вложенных применений правила $\Pi_{n+1}-\mathrm{RR}^{n}$ сводятся к $k+1$ применению правила $\Sigma_{n}$-IR, где первое используется для вывода $I \Sigma_{n-1}$. Вопрос о том, можно ли здесь ограничиться $k$ применениями $\Sigma_{n}$-IR, пока открыт. 
СлЕДСТВИЕ 4.14. $I \Sigma_{n}^{R} \equiv \mathrm{EA}_{\omega}^{n} \equiv I \Pi_{n+1}^{R} \partial$ яя $_{\text {всех } n>0 .}$

Заметим, что это следствие было нами уже доказано для $n=1$ (предложение 3.7 ).

4.4. Беспараметрическая индукция. В этом пункте мы устанавливаем взаимосвязь между схемами рефлексии и схемами беспараметрической индукции в арифметике. Имея в виду точное соответствие схем индукщии и схем равномерной рефлексии над EA (теорема 7), на первый взгляд кажется естественным предположить, что беспараметрическая индукция должна соответствовать схемам локальной рефлексии. Однако нетрудно заметить, что локальные схемы рефлексии являются для этого слишком слабыми принципами: Rfn(EA) содержится в расширении EA множеством всех истинных $\Pi_{1}$-предложений, в то время как по следствиям 4.7, 4.9 и предложению 2.16 ни одна из теорий $I \Pi_{n}^{-}$для $n>1$ в этом расширении не содержится. Оказывается, что для получения точной характеризации необходимо перейти к релятивизированному предикату доказуемости (см. п. 2.3).

Релятивизированные схемы локальной рефлексии определяем по аналогии следуюшим образом:

$$
\begin{aligned}
\operatorname{Rfn}^{n}(T) & :=\left\{[n]_{T} \varphi \rightarrow \varphi \mid \varphi \in S t\right\} \\
\operatorname{Rfn}_{\Sigma_{m}}^{n}(T) & :=\left\{[n]_{T} \sigma \rightarrow \sigma \mid \sigma \in S t \cap \Sigma_{m}\right\} .
\end{aligned}
$$

Для $n=0$ все эти схемы совпадают, по определению, со своими нерелятивизированными аналогами.

Заметим, что по правилу контрапозиции схема $\operatorname{Rfn}_{\Sigma_{m}}^{n}(T)$ эквивалентна над ЕА схеме

$$
\left\{\pi \rightarrow\langle n\rangle_{T} \pi \mid \pi \in S t \cap \Pi_{m}\right\}
$$

Следующая характеризация получена в [16], [18].

ТЕОРема 10. Для $n \geqslant 1$ следуюшие теории эквивалентны:

(i) $I \Sigma_{n}^{-} \equiv \mathrm{EA}+\operatorname{Rfn}_{\Sigma_{n+1}}^{n}(\mathrm{EA})$;

(i) $I \Pi_{n+1}^{-} \equiv \mathrm{EA}+\operatorname{Rfn}_{\Sigma_{n+2}}^{n}(\mathrm{EA})$;

(i) $\mathrm{EA}^{+}+I \Pi_{1}^{-} \equiv \mathrm{EA}^{+}+\mathrm{Rfn}_{\Sigma_{2}}(\mathrm{EA}) \equiv \mathrm{EA}^{+}+\mathrm{Rfn}_{\Sigma_{2}}\left(\mathrm{EA}^{+}\right)$.

ДокАЗАТЕльство. Все утверждения доказьваются аналогично, с использованием теорем 9 (в случае (i)) и 8 (в случаях (ii) и (iii)). Мы приведем лишь доказательстBо (ii).

Для включения $(\subseteq)$ нам необходимо вывести

$$
\varphi(0) \wedge \forall x(\varphi(x) \rightarrow \varphi(x+1)) \rightarrow \forall x \varphi(x)
$$

для каждой $\Pi_{n+1}$-формулы $\varphi(x)$ с единственной свободной переменной $x$. Пусть $\psi$ означает $\Pi_{n+2}$-предложение, логически эквивалентное

$$
\varphi(0) \wedge \forall x(\varphi(x) \rightarrow \varphi(x+1)) .
$$

Как обьчно, внешней индукцией по $m$ легко установить, что для любого $m$ ЕА $+\psi \vdash$ $\varphi(\bar{m})$. Этот факт формализуем в ЕА, поэтому

$$
\mathrm{EA} \vdash \forall x \square_{\mathrm{EA}+\psi} \varphi(\dot{x})
$$


Отсюда мы заключаем, что

$$
\begin{aligned}
\mathrm{EA}+\operatorname{Rfn}_{\Sigma_{n+2}}^{n}(\mathrm{EA})+\psi & \vdash \operatorname{RFN}_{\Pi_{n+1}}(\mathrm{EA}+\psi) \\
& \vdash \forall x\left(\square_{\mathrm{EA}+\psi} \varphi(\dot{x}) \rightarrow \varphi(x)\right) \\
& \vdash \forall x \varphi(x) \quad(\text { по }(4.9)) .
\end{aligned}
$$

Отсюда вытекает

$$
\mathrm{EA}+\operatorname{Rfn}_{\Sigma_{n+2}}^{n}(\mathrm{EA}) \vdash \psi \rightarrow \forall x \varphi(x)
$$

что и требовалось.

Для доказательства включения ( $\supseteq$ ) заметим, что для любого $\Pi_{n+2}$-предложения $\varphi$ теория $I \Pi_{n+1}^{-}+\varphi$ содержит $\mathrm{EA}+\varphi+\Pi_{n+1}$ - IR по лемме 4.6. Следовательно, по теореме 8

$$
I \Pi_{n+1}^{-}+\varphi \vdash\langle n\rangle_{\mathrm{EA}} \varphi \text {. }
$$

Значит,

$$
I \Pi_{n+1}^{-} \vdash \varphi \rightarrow\langle n\rangle_{\mathrm{EA}} \varphi,
$$

откуда вытекает требуемое.

Теорема 10 позволяет применить технику, развитую в п. 2.5, к исследованию беспараметрической индукции. Поскольку релятивизированньй и обычный предикаты доказуемости удовлетворяют условиям Лёба и имеют одинаковую логику доказуемости, все результаты о локальных схемах рефлексии, доказанные нами ранее, обобшаются на релятивизированные схемы.

Аналог предложения 2.33 состоит в следующем.

Лемма 4.15. Любые $m$ примеров схемы $\operatorname{Rfn}^{n}(T) \Pi_{n+1}$-консервативны над $T_{m}^{n}$.

ДокАЗАТЕльство. В доказательстве предложения 2.33 следует читать $[n]_{T}$ всюду вместо $\square_{T}$. Возможность применения леммы 2.27 обеспечивается следствием 2.13 . По лемме 2.9 итерированные формулы $n$-непротиворечивости соответствуют итерированным схемам равномерной $\Pi_{n+1}$-рефлексии над $T$.

Следуюшее предложение получено в [16], [18].

ПРЕДЛОЖЕНИЕ 4.16. Для любого $n \geqslant 1$

(i) любые т примеров схемь $I \Pi_{n+1}^{-} \Pi_{n+1}$-консервативны над $\mathrm{EA}_{m}^{n}$;

(ii) теория $I \Pi_{n+1}^{-} \Pi_{n+1}$-консервативна над $\mathrm{EA}_{\omega}^{n} \equiv I \Sigma_{n}^{R}$.

ДокАЗАТЕльство. Утверждение (ii) следует из (i). Доказательство утверждения (i) основьвается на предыдущей лемме и замечании, что характеризация схем беспараметрической индукции в терминах схем реф̆лексии сохраняет число примеров соответствуюших схем.

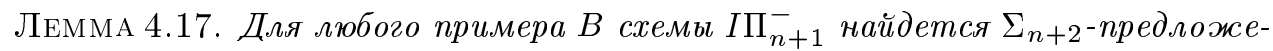
ние $\sigma$ такое, что $[n]_{\mathrm{EA}} \sigma \rightarrow \sigma$ влечет $B$ над ЕА. Обратно, для каждого такого $\sigma$ найдется пример $B$ схемь $I \Pi_{n+1}^{-}$такой, что в $\mathrm{EA}+B$ вьводимо $[n]_{\mathrm{EA}} \sigma \rightarrow \sigma$. 
ДоКАЗАТЕЛЬСТВо получается непосредственной верификацией нашего доказательства теоремы 10. Для доказательства второй части леммы мы используем теорему 8 (i), утверждающую, что

$$
\left[\mathrm{EA}+\neg \sigma, \Pi_{n+1}-\mathrm{IR}\right] \vdash \mathrm{RFN}_{\Pi_{n+1}}(\mathrm{EA}+\neg \sigma) .
$$

Также заметим, что любое конечное число неитерированных применений правила $\Pi_{n+1}$-IR может быть сведено к единственному применению, которое, в свою очередь, сводится к единственному примеру $I \Pi_{n+1}^{-}$.

ЗАмечАниЕ 4.18. Аналогичное утверждение верно для $I \Sigma_{n}^{-}$, но вторая часть имеет место, по-видимому, лишш над $I \Sigma_{n-1}$. Вообще говоря, необходим $m+1$ пример схемы $I \Sigma_{n}^{-}$, чтобы вывести $m$ примеров схемы $\operatorname{Rfn}_{\Sigma_{n+1}}^{n}(\mathrm{EA})$ (первьй из них используется для вывода $\left.I \Sigma_{n-1}\right)$.

В силу леммы 4.17 любые $m$ примеров схемы $I \Pi_{n+1}^{-}$содержатся в некотором расширении ЕА $m$ примерами схемы $\operatorname{Rfn}^{n}(\mathrm{EA})$, которое является $\Pi_{n+1}$-консервативным над $\mathrm{EA}_{m}^{n}$ по лемме 4.15. Это завершает доказательство предложения 4.16.

ЗАмЕчАниЕ 4.19. У тверждение, аналогичное предложению 4.16, также имеет место для схемы $I \Pi_{1}^{-}$над теорией $\mathrm{EA}^{+}$. См. теорему 10 (iii).

Из предложения 4.16 получаем следующее интересное следствие [16], [18].

СлЕДСТВИЕ 4.20. Классы доказуемо тотальньх вычислимых функций теорий $I \Pi_{n+1}^{-}$и I $\Sigma_{n}^{R}$ совпадают. В частности, $\mathscr{F}\left(I \Pi_{2}^{-}\right)$совпадает с множеством всех примитивно рекурсивных функций.

ДокАЗАТЕльство. В силу части (ii) предложения $4.16, I \Pi_{n+1}^{-}$является $\Pi_{n+1}$-консервативным расширением теории $I \Sigma_{n}^{R}$. Класс доказуемо тотальных рекурсивных функций $I \Sigma_{1}^{R}$ совпадает с множеством примитивно рекурсивных функций в силу предложения 3.7 .

Релятивизация теоремы 6 (iii) состоит в следующем.

Лемма 4.21. Для любого $n \geqslant 0$ теория $T+\operatorname{Rfn}^{n}(T)$ консервативна над $T+$ $\operatorname{Rfn}_{\Sigma_{n+1}}^{n}(T)$ относительно $\mathscr{B}\left(\Sigma_{n+1}\right)$-предложсений.

ДокАЗАТЕльство дословно повторяет приведенное нами доказательство теоремы 6.

СЛЕДСТВИЕ 4.22. Для любого $n \geqslant 1$ теория $I \Pi_{n+1}^{-}$консервативна над $I \Sigma_{n}^{-}$ относительно $\mathscr{B}\left(\Sigma_{n+1}\right)$-предложсений.

Из предложения 4.16 также вытекает невозможность конечной аксиоматизации теорий $I \Pi_{n}^{-}$, ранее установленная Р. Кэйе, Дж. Парисом и К. Димитракопулосом [45], использовавшими методы теории моделей. Ниже мы получим обобщение этого результата. Дл этого установим следующий, формально более сильный вариант предложения 4.16 .

ПРЕДЛОЖЕНИЕ 4.23. Пусть T- расширение ЕА конечным числом $\Pi_{n+2}$-nредложений, $n \geqslant 1$. Тогда

(i) расширение $T$ любыми $m$ примерами схемь $I \Pi_{n+1}^{-}$является $\Pi_{n+1}$-консервативным над $T_{m}^{n}$;

(ii) $T+I \Pi_{n+1}^{-}$есть $\Pi_{n+1}$-консервативное расширение $T_{\omega}^{n}$. 
ДокАЗАТЕЛЬСТво. По теореме 10 для данной теории $T$ имеем

$$
\begin{aligned}
T+I \Pi_{n+1}^{-} & \equiv T+\operatorname{Rfn}_{\Sigma_{n+2}}^{n}(\mathrm{EA}) \\
& \equiv T+\operatorname{Rfn}_{\Sigma_{n+2}}^{n}(T)
\end{aligned}
$$

в силу леммы 2.23. По лемме 4.15 отсюда следует второе утверждение. (Тот факт, что $T+I \Pi_{n+1}^{-}$содержит $T_{\omega}^{n}$, вытекает из следствий 4.8 и 4.7.) Первое утверждение получается аналогично из предложения 4.16 (i).

СлЕДСТвИЕ 4.24. Никакая непротиворечивая теория, расширяющая $I \Pi_{n+1}^{-}$ множеством $\Pi_{n+2}$-предложений, не является конечно аксиоматизируемой.

ДокАЗАТЕЛЬСтво. Допустим, от противного, что такое конечное расширение существует. Без ограничения обшности мы можем считать, что оно имеет вид $T+U$ для некоторых $m$ примеров $U$ схемы $I \Pi_{n+1}^{-}$, где $T$ - конечное $\Pi_{n+2}$-аксиоматизированное расширение ЕА. Тогда по предложению 4.23 (i) $\Pi_{n+1}$-следствия теории $T+U$ совпадают с $\Pi_{n+1}$-следствиями $T_{m}^{n}$. Однако по предложению 4.23 (ii)

$$
T+I \Pi_{n+1}^{-} \vdash \operatorname{RFN}_{\Pi_{n+1}}\left(T_{m}^{n}\right) .
$$

Последняя формула является $\Pi_{n+1}$-формулой и невыводима в $T_{m}^{n}$.

Отметим также следующее следствие теоремы о неограниченности для локальных схем рефилексии.

ПрЕДЛОЖЕНИЕ 4.25. $\Pi_{n+1}^{-}$не содержится ни в каком непротиворечивом перечислимом расширении ЕА множеством $\Pi_{n+2}-$-преложений.

ДокАЗАТЕЛЬСтво. По теореме $10 I \Pi_{n+1}^{-}$содержит схему $\operatorname{Rfn}_{\Sigma_{n+2}}^{n}(\mathrm{EA})$ и, тем самым, более слабую схему $\operatorname{Rfn}_{\Sigma_{n+2}}(\mathrm{EA})$. Результат получается применением следствия 2.22 .

СлеДСТВИЕ 4.26. $I \Pi_{n+1}^{-} \nsubseteq I \Sigma_{n+1}^{R}$.

Заметим, что сложность естественной аксиоматизации $I \Pi_{n+1}^{-}$есть $\Sigma_{n+2}$, а сложность $I \Sigma_{n}^{-}$есть $\mathscr{B}\left(\Sigma_{n+1}\right)$. Мы имеем следуюший вариант теоремы о неограниченности для $\operatorname{Rfn}_{\Sigma_{n+1}}^{n}(T)$.

Лемма 4.27. $\operatorname{Rfn}_{\Sigma_{n+1}}^{n}(T)$ не содержится ни в каком непротиворечивом конечном расширении теории $T$ с помощью $\mathscr{B}\left(\Sigma_{n+1}\right)$-предложений.

ДокАЗАТЕЛЬСТво. Очевидная релятивизация леммы 2.26 показывает, что схемы $\operatorname{Rfn}_{\Sigma_{n+1}}^{n}(T)$ и $\operatorname{Rfn}_{\mathscr{B}\left(\Sigma_{n+1}\right)}^{n}(T)$ дедуктивно эквивалентны над ЕА. Если последняя содержится в теории $T+\varphi$, где $\varphi \in \mathscr{B}\left(\Sigma_{n+1}\right)$, то $T+\varphi \vdash[n]_{T} \neg \varphi \rightarrow \neg \varphi$, и тем более $T \vdash \square_{T} \neg \varphi \rightarrow \neg \varphi$. Отсюда по теореме Лёба $T \vdash \neg \varphi$.

Как следствие получаем следуюшее предложение.

ПредЛОЖЕНИЕ 4.28. I $\Sigma_{n}^{-}$не содержится ни в каком непротиворечивом конечном расширении ЕА с помощью $\mathscr{B}\left(\Sigma_{n+1}\right)$-предложений. 
СЛЕДСТВИЕ 4.29. Любая непротиворечивая теория, расширяющая $I \Sigma_{n}^{-}$с помощью $\mathscr{B}\left(\Sigma_{n+1}\right)$-предложений, не является конечно аксиоматизируемой.

ДокАЗАТЕЛЬСТво. Это вытекает из предложения 4.28 и того, что сама теория $I \Sigma_{n}^{-}$имеет $\mathscr{B}\left(\Sigma_{n+1}\right)$-аксиоматизацию.

Следующее утверждение ранее доказано в [45] другим методом.

С ЛЕДСТВИЕ 4.30. Для любого $n \geqslant 1$ теории $I \Sigma_{n}^{-}$и $\Pi_{n}^{-}$не являются конечно аксиоматизируемыми.

ДокАЗАТЕЛЬСТвО. Утверждение вытекает из следствий 4.29 и 4.24. Для случая $I \Pi_{1}^{-}$достаточно заметить, что если эта теория конечно аксиоматизируема, то таковой является и теория $\mathrm{EA}^{+}+I \Pi_{1}^{-}$, что невозможно в силу замечания 4.19 .

4.5. Схемы и правила рефлексии. В этом пункте мы устанавливаем взаимосвязь между схемами и правилами рефлексии в арифметике. Мы показьваем, что при определенных условиях схема $\operatorname{RFN}_{\Pi_{n+2}}(T)$ является $\Pi_{n+1}$-консервативным расширением замыкания теории $T$ относительно правила $\Pi_{n+1^{-}} \operatorname{RR}^{n}(T)$, т.е. теории $T_{\omega}^{n}$.

Для $T=$ PRA этот результат доказал У. Шмерль [77]. Однако для дальнейшего будет важно, что это утверждение имеет место при существенно более широких предположениях относительно теории $T$. Как мы покажем, из этого обобщения можно вьвести ряд известных результатов: теорему Парсонса о частичной консервативности схем над правилами индукции и теорему Кэя, Париса и Димитракопулоса о частичной консервативности параметрических схем индукции над беспараметрическими. Мы также получаем следствие о $\Sigma_{2}$-консервативности $\operatorname{RFN}_{\Sigma_{1}}(T)$ над $\operatorname{Rfn}_{\Sigma_{1}}(T)$.

Доказываемая нами теорема [19] играет важную роль в следующем параграфе, где на ее основе с использованием техники алгебр доказуемости получается новое доказательство непротиворечивости РА в стиле Генщена и дается характеризация доказуемо тотальных вычислимых функций РА.

ТЕОРема 11. Пусть T - әлементарно аксиоматизированная теория, содержащая $\mathrm{EA}$, а $U$ - любое $\Pi_{n+2}$-аксиоматизированное расширение $\mathrm{EA}$. Тогда теория $U+\operatorname{RFN}_{\Sigma_{n+1}}(T) \Pi_{n+1}$-консервативна над $U+\Pi_{n+1}-\operatorname{RR}^{n}(T)$.

ДокАЗАТЕльство. Для любого терма $s$ и формулы $\varphi(a)$ обозначим через $\ulcorner\psi(\dot{s})\urcorner$ результат подстановки $s$ в терм $\ulcorner\psi(\dot{a})\urcorner$.

ЛЕмма 4.31. Для любого терма $s(\vec{x})$, где список $\vec{x}$ исчерпьвает все переменнье терма $s$, июбой формуль $\varphi($ а) (где $s$ подстановочен в ч вместо а)

$$
\mathrm{EA} \vdash \forall \vec{x} \quad\left(\square_{T} \varphi(s(\dot{\vec{x}})) \leftrightarrow \square_{T} \varphi(\dot{s})\right) .
$$

ДокАЗАТЕЛЬСтво. Очевидно,

$$
\mathrm{EA} \vdash s(\vec{x})=y \rightarrow(\varphi(s(\vec{x})) \leftrightarrow \varphi(y))
$$

Отсюда, в силу доказуемой $\Sigma_{1}$-полноты и условий Лёба,

$$
\begin{aligned}
\mathrm{EA} \vdash s(\vec{x})=y & \rightarrow \square_{T}(s(\dot{\vec{x}})=\dot{y}) \\
& \rightarrow \square_{T}(\varphi(s(\dot{\vec{x}})) \leftrightarrow \varphi(\dot{y})) \\
& \rightarrow\left(\square_{T} \varphi(s(\dot{\vec{x}})) \leftrightarrow \square_{T} \varphi(\dot{y})\right) .
\end{aligned}
$$


С другой стороны, по определению $\ulcorner\varphi(\dot{s})\urcorner$,

$$
\mathrm{EA} \vdash s(\vec{x})=y \rightarrow\left(\square_{T} \varphi(\dot{s}) \leftrightarrow \square_{T} \varphi(\dot{y})\right),
$$

что вместе с (4.10) дает требуемое.

Мы покажем, что стандартная процедура устранения сечения может рассматриваться как редукция $\operatorname{RFN}_{\Sigma_{n+1}}(T)$ к $\Pi_{n+1}-\mathrm{RR}^{n}(T)$. Рассмотрим вывод без сечений секвенции вида

$$
\neg U, \quad \neg \operatorname{RFN}_{\Sigma_{n+1}}(T), \quad \Pi,
$$

где П есть множество $\Pi_{n+1}$-формул, $\neg U$ есть конечное множество отрицаний аксиом теории $U$, a $\neg \operatorname{RFN}_{\Sigma_{n+1}}(T)$ - конечное множество отрицаний примеров схемы $\operatorname{RFN}_{\Sigma_{n+1}}(T)$ вида

$$
\exists y \exists x\left[\operatorname{Prf}_{T}(y,\ulcorner\neg \varphi(\dot{x})\urcorner) \wedge \varphi(x)\right]
$$

для некоторых формул $\varphi(x) \in \Pi_{n+1}$. Обозначим формулу в квадратных скобках через $R_{\varphi}(x, y)$. Мы также можем считать, что аксиомы $U$ имеют вид $\forall x_{1} \ldots \forall x_{m}$ $\neg A\left(x_{1}, \ldots, x_{m}\right)$ для некоторых $\Pi_{n+1}$-формул $A(\vec{x})$.

По свойству подформульности любая формула, входящая в вьвод секвенщии $\Gamma$ вида (4.11), либо (а) является $\Pi_{n+1}$-формулой, либо (b) имеет вид $\neg \operatorname{RFN}_{\Sigma_{n+1}}(T)$, $\exists x R_{\varphi}(t, x)$ или $R_{\varphi}(t, s)$ для некоторых термов $s, t$, либо (с) имеет вид

$$
\exists x_{i+1} \ldots \exists x_{n} A\left(t_{1}, \ldots, t_{i}, x_{i+1}, \ldots, x_{m}\right)
$$

для некоторого $i<m$ и термов $t_{1}, \ldots, t_{i}$. Обозначим через $\Gamma^{-}$результат вычеркивания всех формул типа (b) и (c) из секвенции $\Gamma$.

Лемма 4.32. Если секвениия Г вида (4.11) доказуема без сечения, то $\bigvee \Gamma^{-}$ доказуема в теории $U+\Pi_{n+1}-\operatorname{RR}^{n}(T)$.

ДОКАЗАТЕЛЬСТВО Проходит с помошью индукции по высоте вьвода $d$ секвенции $\Gamma$. Достаточно рассмотреть случай, когда формула вида (b) или (c) вводится последним применением правила в $d$, и к тому же достаточно рассмотреть лишш формулы вида $R_{\varphi}(t, s)$ и $\exists x_{m} A\left(t_{1}, \ldots, t_{m-1}, x_{n}\right)$, поскольку в остальных случаях после применения операции $(\cdot)^{-}$посылка и заключение правила совпадают.

Таким образом, допустим, что вьвод $d$ имеет вид

$$
\frac{\operatorname{Prf}_{T}(t,\ulcorner\neg \varphi(\dot{s})\urcorner), \Delta \quad \varphi(s), \Delta}{R_{\varphi}(t, s), \Delta}(\wedge),
$$

где $\varphi \in \Pi_{n+1}$. Тогда по предположению индукции мы получаем выводы в $U+\Pi_{n+1}-\mathrm{RR}^{n}(T)$ формул

$$
\operatorname{Prf}_{T}(t,\ulcorner\neg \varphi(\dot{s})\urcorner) \vee \bigvee \Delta^{-}
$$

и

$$
\varphi(s) \vee \bigvee \Delta^{-}
$$

Поскольку $\Delta^{-}$состоит из $\Pi_{n+1}$-формул, правило $\Pi_{n+1}-\mathrm{RR}^{n}(T)$ применимо к (4.13), и мы получаем вывод

$$
\langle n\rangle_{T}\left(\varphi(s(\dot{\vec{x}})) \vee \bigvee \Delta^{-}(\dot{\vec{x}})\right) .
$$


Отсюда, пользуясь условиями Лёба, мы последовательно выводим:

1) $\langle n\rangle_{T} \bigvee \Delta^{-}(\dot{\vec{x}}) \vee\langle n\rangle_{T} \varphi(s(\dot{\vec{x}}))$,

2) $\langle n\rangle_{T}\left(\bigvee \Delta^{-}(\overrightarrow{\vec{x}})\right) \rightarrow \bigvee \Delta^{-}(\vec{x})$ (по доказуемой $\Sigma_{n+1}$-полноте),

3) $\bigvee \Delta^{-}(\vec{x}) \vee\langle n\rangle_{T} \varphi(s(\overrightarrow{\vec{x}}))$ (из 1), 2) и условий Лёба),

4) $\bigvee \Delta^{-}(\vec{x}) \vee\langle n\rangle_{T} \varphi(\dot{s}) \quad$ (по лемме 4.31).

$\mathrm{C}$ другой стороны, из (4.12), заменяя терм $t$ на квантор сушествования и ослабляя $\square_{T}$ до $[n]_{T}$, получаем

$$
\bigvee \Delta^{-}(\vec{x}) \vee[n]_{T} \neg \varphi(\dot{s}) .
$$

Вместе с 4) по правилу сечения это дает вывод формулы $\bigvee \Delta^{-}$в теории $U+$ $\Pi_{n+1}-\mathrm{RR}^{n}(T)$.

Если последнее применение правила в выводе $d$ имеет вид

$$
\frac{A\left(t_{1}, \ldots, t_{m-1}, t_{m}\right), \Delta}{\exists x_{n} A\left(t_{1}, \ldots, t_{m-1}, x_{m}\right), \Delta}(\exists),
$$

то по предположению индукции имеем вьвод

$$
A\left(t_{1}, \ldots, t_{m-1}, t_{m}\right) \vee \bigvee \Delta^{-}
$$

в $U+\Pi_{n+1}-\mathrm{RR}^{n}(T)$. Отсюда выводим

$$
\exists x_{1} \ldots \exists x_{m} A\left(x_{1}, \ldots, x_{m}\right) \vee \bigvee \Delta^{-},
$$

и $\bigvee \Delta^{-}$следует по правилу сечения с аксиомой $\forall x_{1} \ldots \forall x_{n} \neg A\left(x_{1}, \ldots, x_{m}\right)$ теории $U$.

СлЕДСТВИЕ 4.33. Если T- $\Pi_{n+2}$-аксиоматизированное расширение ЕА, то теория $T+\operatorname{RFN}_{\Sigma_{n+1}}(T)$ есть $\Pi_{n+1}$-консервативное расширение $T_{\omega}^{n}$.

Отметим, что это утверждение для $T=$ PRA другим методом получено в работе [77], а для $n=1$ и $T=$ ЕА в [13]. Если $T=\mathrm{EA}$, то отсюда вытекает следуюший результат Ч. Парсонса [66].

ПРЕДЛОЖЕНИЕ 4.34. Для $n \geqslant 1$ теория $I \Sigma_{n} \Pi_{n+1}$-консервативна над $I \Sigma_{n}^{R}$. В частности, I $\Sigma_{1} \Pi_{2}$-консервативна над $I \Sigma_{1}^{R} \equiv \operatorname{PRA}$ и $\mathscr{F}\left(I \Sigma_{1}\right)$ есть класс примитивно рекурсивных функиии.

Мы также имеем более сильньй результат о консервативности для беспараметрических схем.

ЛЕмма 4.35. Если $U-\Pi_{n+2}$-аксиоматизированное расширение ЕА, то теория $U+\operatorname{RFN}_{\Sigma_{n+1}}(T)$ есть $\Sigma_{n+2}$-консервативное расширение $U+\operatorname{Rfn}_{\Sigma_{n+1}}^{n}(T)$.

В частности, для $n=0$ и $U=$ ЕА получаем, что схема $\operatorname{RFN}_{\Sigma_{1}}(T) \Sigma_{2}$-консервативна над $\operatorname{Rfn}_{\Sigma_{1}}(T)$.

ДокАЗАТЕльСтво. Допустим $U+\operatorname{RFN}_{\Sigma_{n+1}}(T) \vdash \sigma$ для некоторого предложения $\sigma \in \Sigma_{n+2}$, тогда $U+\neg \sigma+\operatorname{RFN}_{\Sigma_{n+1}}(T) \vdash \perp$, и по теореме $11 U+\neg \sigma+\Pi_{n+1}-\operatorname{RR}^{n}(T) \vdash \perp$. Отсюда мы получаем $U+\neg \sigma+\operatorname{Rfn}_{\Sigma_{n+1}}^{n}(T) \vdash \perp$, и по теореме о дедукции $U+\operatorname{Rfn}_{\Sigma_{n+1}}^{n}(T)$ $\vdash \sigma$.

С учетом теоремы 10 отсюда непосредственно вытекает следующее предложение, доказанное Р. Кэйем, Дж. Парисом и К. Димитракопулосом [45] методами теории моделей. 
ПРЕДЛОЖЕНИЕ 4.36. Для $n \geqslant 1$ теория $I \Sigma_{n}$ является $\Sigma_{n+2}$-консервативным расиирением $I \Sigma_{n}^{-}$.

В заключение отметим ряд фактов, относяшихся к диаграмме фрагментов РА на рис. 2 (п. 3.1).

Так, $I \Pi_{n+1}^{-} \nsubseteq I \Sigma_{n+1}^{R}$ вытекает из следствия 4.26. $I \Sigma_{n} \nsubseteq I \Pi_{n+1}^{-}$следует из того, что $I \Pi_{n+1}^{-}$имеет $\Sigma_{n+2}$-аксиоматизацию, а $I \Sigma_{n}$ содержит $\operatorname{RFN}_{\Pi_{n+2}}$ (EA) по теореме 7 . $I \Sigma_{n+1}^{R} \not \subseteq I \Sigma_{n}+I \Pi_{n+1}^{-}$следует из того, что $I \Sigma_{n}+I \Pi_{n+1}^{-}$есть расширение $I \Sigma_{n}$ множеством $\Sigma_{n+2}$-предложений, в то время как $I \Sigma_{n+1}^{R}$ содержит $\operatorname{RFN}_{\Pi_{n+2}}\left(I \Sigma_{n}\right)$. Таким образом, все включения, соответствуюшие ребрам диаграммы, являются строгими.

\section{§5. Алгебры доказуемости и ординальный анализ РА}

В предыдущем параграфе мы показали, что различные фрагменты РА выражаются тем или иным образом через схемы рефлексии над элементарной арифметикой. Общие свойства схем рефлексии затем позволяют сравнительно легко установить самые разные характеристики изучаемых теорий. Таким образом, структура схем рефлексии над данной теорией $T$ выступает как своего рода "карта" и инструмент исследования расширений $T$.

Структура схем рефлексии над $T$ и объемлюшая ее структура произвольных расширений $T$ могут быть осмыслены с достаточно обшей алгебраической точки зрения. Эта точка зрения оказьвается плодотворной для дальнейших приложений схем рефлексии к ординальному анализу и общим вопросам классификации теорий. Мы начнем с известного понятия алгебры Линденбаума теории.

5.1. Алгебры Линденбаума. Для данной теории $T$ рассмотрим множество $\mathscr{L}_{T}$ всех арифметических предложений, факторизованное отношением доказуемой эквивалентности в $T$ :

$$
\varphi \sim \psi \Longleftrightarrow T \vdash \varphi \leftrightarrow \psi
$$

Класс эквивалентности формулы $\varphi$ обозначаем $\{\varphi\}$. Логические операции $\wedge, \vee, \neg$ вместе с отношением порядка

$$
\{\varphi\} \leqslant\{\psi\} \Longleftrightarrow T \vdash \varphi \rightarrow \psi
$$

наделяют это множество структурой булевой алгебры, называемой алгеброй Линденбаума теории $T$. Наибольший $\top$ и наименьший $\perp$ элементы этой алгебры составляют соответственно классы всех доказуемых и всех опровержимых в $T$ предложений. Остальные элементы алгебры Линденбаума представляют собой классы эквивалентности $T$-независимых утверждений. Дедуктивно замкнутые расширения $T$ соответствуют фильтрам булевой алгебры $\mathscr{L}_{T}$.

Напомним, что булева алгебра $\mathscr{B}$ назьвается безатомной, если

$$
\forall x, y \in \mathscr{B}(x<y \Rightarrow \exists z x<z<y) \text {. }
$$

Теорема Россера влечет следующее утверждение.

ПРЕДЛОЖЕНИЕ 5.1. Пусть T - перечислимая непротиворечивая теория. Тогда булева алгебра $\mathscr{L}_{T}$ счетна и безатомна. 
ДокаЗАтельство. Рассмотрим $\varphi$ и $\psi$ такие, что $\{\varphi\}<\{\psi\}$, т.е. $T \vdash \varphi \rightarrow \psi$ и $T \nvdash \psi \rightarrow \varphi$. Тогда теория $U=T+\psi+\neg \varphi$ непротиворечива. По теореме 3 найдется предложение $\theta$, независимое от $U$. В качестве $z$ возьмем класс $\{\varphi \vee(\theta \wedge \psi)\}$.

Из элементарной теории булевых алгебр (см. [37]) известно, что все счетные безатомные булевы алгебры изоморфны.

СлЕДСТвИЕ 5.2. Если теории $T$ и $U$ перечислимы и непротиворечивы, то $\mathscr{L}_{T} \simeq \mathscr{L}_{U}$.

В частности, изоморфными являются алгебры $\mathscr{L}_{\text {EA }}, \mathscr{L}_{\mathrm{PA}}, \mathscr{L}_{\text {ZF }}$ и т. д. Справедливо даже более сильное утверждение.

Алгебру $\mathscr{L}_{T}$ можно рассматривать как (позитивно) нумерованную структуру, где гёделевы номера формул выступают как коды элементов алгебры (см. [30]). С. Крипке и М.Б. Пур-Эль [69] доказали, что все алгебры Линденбаума для непротиворечивых перечислимых арифметических теорий, рассматриваемые как нумерованные алгебры, являются рекурсивно изоморфными. Фактически, это утверждение следует из эффективности теоремы Россера.

5.2. Алгебры и логики доказуемости. Приведенный вьше результат об изоморфизме говорит о том, что алгебра Линденбаума не несет интересной с точки зрения теории доказательств информации о "силе" формальной системы. Возникает вопрос, нельзя ли обогатить эту структуру таким образом, чтобы интересующие нас свойства теорий стали выразимыми.

А. Тарский (см. [40]) ввел понятие цилиндрической алгебры, которое фактически позволяет выразить кванторы на алгебраическом языке. Однако, поскольку язык цилиндрических алгебр имеет очень большую выразительную силу, соответствующие структуры слишком сложны и, по ви-димому, не дают особых преимушеств для теории доказательств по сравнению с традиционным языком логики первого порядка.

Другое обогашение структуры алгебры Линденбаума было предложено Р. Магари [55]. Для данной элементарно аксиоматизированной теории $T$ гёделевская формула доказуемости индуцирует оператор

$$
\square_{T}:\{\varphi\} \longmapsto\left\{\square_{T} \varphi\right\},
$$

действующий на алгебре $\mathscr{L}_{T}$. В силу условий Лёба этот оператор корректно определен на классах эквивалентности. Структура $\mathscr{M}_{T}=\left(\mathscr{L}_{T}, \square_{T}\right)$ назьвается алгеброй доказуемости или алгеброй Магари для теории $T{ }^{4}$

Язык алгебр Магари позволяет выразить многие интересные факты о доказуемости, например, вторую теорему Гёделя о неполноте:

$$
\mathscr{M}_{T} \vDash \forall x\left(x \neq \perp \Rightarrow x \nless \neg \square_{T} \neg x\right) .
$$

Заметим, что термы язька алгебр Магари отождествляются с пропозициональными модальными формулами. В соответствии с этим теорема Соловея описывает все термы $\varphi(\vec{x})$ такие, что в $\mathscr{M}_{T}$ выполнено тождество $\varphi(\vec{x})=\top$ :

$$
\mathbf{G L} \vdash \varphi(\vec{x}) \Longleftrightarrow \mathscr{M}_{T} \vDash \forall \vec{x} \varphi(\vec{x})=\top .
$$

\footnotetext{
${ }^{4}$ Сам Р. Магари назьвал такие алгебры диагонализируемыми .
} 
Произвольное тождество $\psi(\vec{x})=\theta(\vec{x})$ алгебры доказуемости сводится к тождеству вида $\varphi(\vec{x})=\top$, где $\varphi=(\psi \leftrightarrow \theta)$. Таким образом, с алгебраической точки зрения теорема Соловея представляет собой характеризацию тождеств алгебр доказуемости для $\Sigma_{1}$-корректных теорий $T$.

Булеву алгебру с одноместньм оператором $\square$, удовлетворяющую тождествам

M1. $\square(\varphi \rightarrow \psi) \rightarrow(\square \varphi \rightarrow \square \psi)=\top$,

M2. $\square \varphi \rightarrow \square \square \varphi=\top$,

M3. $\square(\square \varphi \rightarrow \varphi) \rightarrow \square \varphi=\top$,

M4. $\square \top=\top$

и тем самым всем теоремам логики GL, назьваем алгеброй Магари. По теореме Соловея совокупность алгебр Магари есть многообразие, порожденное алгеброй $\mathscr{M}_{T}$ для какой-нибудь $\Sigma_{1}$-корректной теории $T$, например PА.

Общие свойства алгебр Магари активно изучались в контексте логики доказуемости (см. [55], [56], [59]-[61], [86], [6]). С точки зрения исследования структуры алгебр Магари арифметических теорий наибольший интерес представляют результаты В. Ю. Шаврукова (см. [80]-[83], а также [95]). В частности, Шавруков дал описание возможных подалгебр алгебры $\mathscr{M}_{T}$. Им был также установлен важный результат о неразрешимости элементарной теории алгебры $\mathscr{M}_{T}$. Отметим, что разрешимость универсальной теории этой алгебры вытекает из разрешимости истинностной логики доказуемости $\mathbf{S}$ (см. [6]), в то время как разрешимость $\forall^{*} \exists^{*}$-теории представляет собой трудньй открытьй вопрос. Шавруков также установил ряд интересных результатов об изоморфизме алгебр доказуемости. В частности, он доказал, что алгебры $\mathscr{M}_{T}$ и $\mathscr{M}_{U}$ не являются изоморфньми, если $U \vdash \mathrm{RFN}_{\Sigma_{1}}(T) .{ }^{5}$ Вопрос об изоморфизме алгебр таких теорий, как РА и PA + Con(PA), пока открыт.

5.3. Градуированные алгебры доказуемости и логика Джапаридзе. Формула доказуемости имеет логическую сложность $\Sigma_{1}$. В то же время интересующие нас теории (такие, как $I \Sigma_{1}$ или РА) часто имеют более высокий, или даже неограниченньй, уровень арифметической сложности аксиом. Это наводит на мысль, что язык алгебр Магари еще недостаточно выразителен для представления и исследования свойств таких теорий.

Нужное обогащение дают формулы $n$-доказуемости $[n]_{T}$ и соответствуюшие операторы на алгебре $\mathscr{M}_{T}$. Это приводит к понятию градуированной алгебры доказуемости, введенном в [21]. В предыдушем параграфе мы видели, что уже простое использование формулы $[n]_{T}$ как модальности логики GL позволяет получить ряд нетривиальных результатов о схемах беспараметрической индукции. Совместное использование нескольких операторов доказуемости позволяет продвинуться далее в исследовании арифметических теорий и получить такие результаты, как знаменитую теорему Генцена о доказательстве непротиворечивости РА с помошью трансфинитной индукции до ординала $\varepsilon_{0}$, характеризацию доказуемо тотальных вычислимых функций в РА, и приводит к простым утверждениям комбинаторного характера, независимым от арифметики Пеано.

\footnotetext{
${ }^{5}$ В. Ю. Шавруков использовал несколько более жесткое условие, которое эквивалентно данному в силу некоторых результатов [14].
} 
Каждая формула $[n]_{T}$ задает на алгебре Линденбаума теории $T$ оператор

$$
[n]_{T}:\{\varphi\} \longmapsto\left\{[n]_{T} \varphi\right\} .
$$

Мын называем алгебру $\mathscr{M}_{T}^{\infty}=\left(\mathscr{L}_{T},[0]_{T},[1]_{T}, \ldots\right)$ әрадуированной алгеброй доказуемости для теории $T$. Мы будем также иногда рассматривать $\mathscr{M}_{T}^{\infty}$ как многосортную алгебру, т.е. структуру с выделенньм семейством подмножеств

$$
\Pi_{1} \subset \Pi_{2} \subset \Pi_{3} \subset \cdots \subset \mathscr{M}_{T}^{\infty}
$$

определяемых предложениями соответствуюшего уровня арифметической иерархии.

В качестве наиболее важного примера рассмотрим алгебру $\mathscr{M}_{\mathrm{EA}}^{\infty}$. Знакомые нам фрагменты РА соответствуют фильтрам этой алгебры, порождаемым некоторыми специфическими элементами. Так, в силу результатов предыдущего параграффа ограниченная схема индукции $I \Sigma_{n}$ соответствует элементу $\langle n+1\rangle_{\text {ЕА }} \top$ (и порождаемому им фильтру).

Правилам $\Sigma_{n^{-}}$и $\Pi_{n+1}$-индукции отвечает оператор $p \mapsto\langle n\rangle_{\text {ЕА }} p$ для $p \in \Pi_{n+1}$ и $p \in \Pi_{n+2}$ соответственно. Таким образом, $I \Pi_{n+1}^{R}$ есть фильтр, порожденный из $\top$ этим оператором, т.е. замыкание $\left\{\langle n\rangle_{\text {ЕА }}^{k} \top: k<\omega\right\}$.

Схемы беспараметрической индукции суть множества элементов $\mathscr{M}_{\mathrm{EA}}^{\infty}$ вида $p \rightarrow$ $\langle n\rangle_{\text {ЕА }} p$, где $p \in \Pi_{n+1}$ и $p \in \Pi_{n+2}$ соответственно.

Термы алгебры $\mathscr{M}_{T}^{\infty}$ отождествляются с пропозищиональными полимодальным.ми формулами с модальностями $[0],[1],[2]$, . . Тождества этой алгебры задаются следуюшей логикой GLP, введенной Г. К. Джапаридзе [43], [42].

\section{Аксиомы:}

J1. Аксиомы GL для каждой модальности $[n]$;

J2. $[m] \varphi \rightarrow[n] \varphi$ для $m \leqslant n ;$

J3. $\langle m\rangle \varphi \rightarrow[n]\langle m\rangle \varphi$ для $m<n$.

Правила вывода: modus ponens, $\varphi \vdash[n] \varphi$.

Арифметическая интерпретация $(\varphi)_{T}^{*}$ полимодальной формулы $\varphi$ определена как обычно, при этом для всех $n[n]$ понимается как $n$-доказуемость:

$$
([n] \varphi)_{T}^{*}=[n]_{T}(\varphi)_{T}^{*}
$$

Лемма 5.3. Если GLP $\vdash \varphi$, то ЕА $\vdash(\varphi)_{T}^{*}$ для любой реализации $(\cdot)^{*}$ переменных $\varphi$.

ДокАЗАТЕЛЬСТВО. Корректность аксиом логики GL следует из справедливости условий и теоремы Лёба для формул $n$-доказуемости. Справедливость аксиомы J3 вытекает из доказуемой $\Sigma_{n+1}$-полноты. Аксиома J2 следует из верифицируемости в ЕА того тривиального факта, что всякая (истинная) $\Pi_{n}$ формула является (истинной) $\Pi_{n+1}$-формулой.

По аналогии с алгебрами Магари мы называем алгебрами Джапаридзе булевы алгебры с операторами $[0],[1],[2], \ldots$, удовлетворяющие всем тождествам GLP. Г. К. Джапаридзе [43], [42] получил следующее глубокое обобщение теоремы Соловея об арифметической полноте. 
ТЕОРема 12. Если теория Т корректна, то для любой полимодальной форму$л b l \varphi$

$$
\operatorname{GLP} \vdash \varphi(\vec{x}) \Longleftrightarrow \mathscr{M}_{T}^{\infty} \vDash \forall \vec{x}(\varphi(\vec{x})=\top) .
$$

Таким образом, многообразие алгебр Джапаридзе порождается алгеброй $\mathscr{M}_{T}^{\infty}$ для любой корректной теории $T$. Отметим, что в этой теореме условие корректности $T$ является необходимым.

Первоначально Г. К. Джапаридзе получил свой результат для несколько иной интерпретации модальностей $[n]$. История вопроса такова.

Дж. Булос [24] исследовал модальную логику $\omega$-дожазуемости (понятие, двойственное $\omega$-непротиворечивости по Гёделю) и установил, что теоремы Соловея переносятся на этот случай без больших изменений. $\omega$-доказуемость может быть описана как вьводимость с помощью одного применения $\omega$-правила, т.е. как доказуемость в теории

$$
T^{\prime}:=T+\{\forall x \varphi(x): \forall n \in \mathbb{N} T \vdash \varphi(\bar{n})\}
$$

Таким образом, логика $\omega$-доказуемости совпадает с GL.

Г.К. Джапаридзе [42] получил более интересную характеризацию совместной логики доказуемости и $\omega$-доказуемости для РА. Фактически, он сформулировал полимодальную логику GLP для интерпретации [1], [2], . . как формул доказуемости в теориях PA', PA" и т. д. В дальнейшем К. Н. Игнатьев [41] упростил работу Г. К. Джапаридзе и подробно исследовал модально-логические свойства GLP. Он также заметил, что теорема Джапаридзе имеет место и при более общих условиях на последовательность формул, интерпретирующих модальности $[n]$. В частности, арифметическая полнота GLP установлена и для интерпретации модальностей как формул $n$-доказуемости. Градуированные алгебры доказуемости введены в [21], где была обнаружена их связь с ординальным анализом арифметики Пеано.

Логика Джапаридзе разрешима и может быть исследована с помощью семантики Крипке. Строго говоря, GLP не является полной по Крипке, однако она имеет простой перевод в более слабую логику $\mathbf{G L P} \mathbf{P}^{-}$, получаемую из GLP путем замены аксиомы J2 на

$$
[m] \varphi \rightarrow[n][m] \varphi \text { для } m \leqslant n .
$$

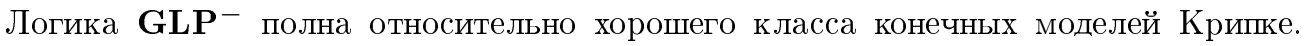
Игнатьев также получил теорему о нормальной форме для замкнутых формул логики GLP, которые играют большую роль в дальнейшем. Подробное изложение логики Джапаридзе дано в [25], [41]. В данной работе нам понадобится лишш арифметическая корректность GLP, выражаемая леммой 5.3.

5.4. Свойство редукции. Доказанная нами в п. 4.5 теорема 11 выражает важное свойство градуированных алгебр доказуемости. Пусть $U$ и $V$ - подмножества $\mathscr{M}_{T}^{\infty}$. Пишем $U \equiv_{n} V$, если порождаемые ими фильтры (т.е. теории, содержащие $T$ ) взаимно $\Pi_{n+1}$-консервативны.

ПРЕДЛОЖЕНИЕ 5.4. Пусть теория Т есть П ${ }_{n+2}$-расширение ЕА. Тогда для любого $\varphi \in \mathscr{M}_{T}^{\infty}$

$$
\left\{\langle n+1\rangle_{T} \varphi\right\} \equiv_{n}\left\{Q_{k}^{n}(\varphi): k<\omega\right\},
$$


$2 \partial e$

$$
\begin{aligned}
Q_{0}^{n}(\varphi) & =\langle n\rangle_{T} \varphi \\
Q_{k+1}^{n}(\varphi) & =\langle n\rangle_{T}\left(Q_{k}^{n}(\varphi) \wedge \varphi\right) .
\end{aligned}
$$

ДоКАЗАТЕльство. Утверждение получается, если в теореме 11 взять теорию $T$ в качестве $U$ и $T+\varphi$ в качестве $T$. Заметим, что правило $\Pi_{n+1}-\mathrm{RR}^{n}(T+\varphi)$ эквивалентно правилу

$$
\frac{\psi}{\langle n\rangle_{T}(\varphi \wedge \psi)}, \quad \psi \in \Pi_{n+1}
$$

Теория $T+\left\{Q_{k}^{n}(\varphi): k<\omega\right\}$ представляет собой замыкание Т относительно этого правила. В самом деле, если $T+Q_{k}^{n}(\varphi) \vdash \psi$, то

$$
\begin{aligned}
T+Q_{k+1}^{n}(\varphi) & \vdash\langle n\rangle_{T}\left(\varphi \wedge Q_{k}^{n}(\varphi)\right) \\
& \vdash\langle n\rangle_{T}(\varphi \wedge \psi) .
\end{aligned}
$$

Таким образом, все $\Pi_{n+1}$-следствия элемента $\langle n+1\rangle_{T} \varphi \in \mathscr{M}_{T}^{\infty}$, имеющего сложность $\Pi_{n+2}$, порождаются $\Pi_{n+1}$-элементами $Q_{k}^{n}(\varphi)$. Мы назьваем это свойство алгебры $\mathscr{M}_{T}^{\infty}$ свойством редукиии. В дальнейшем играет сушественную роль то обстоятельство, что элементы $Q_{k}^{n}(\varphi)$ выражаются через операции алгебры $\mathscr{M}_{T}^{\infty}$.

В качестве примера возьмем $\varphi=\top$ в формулировке предложения 5.4. Тогда мы получим

$$
\left\{\langle n+1\rangle_{T}^{\top}\right\} \equiv_{n}\left\{\langle n\rangle_{T}^{k+1} \top: k<\omega\right\} .
$$

Нетрудно видеть, что это утверждение выражает следствие 4.33 на языке градуированных алгебр доказуемости.

СлЕДСТВИЕ 5.5. Если $m \leqslant n, m o$

$$
\mathrm{EA}^{+} \vdash\langle m\rangle\langle n+1\rangle \varphi \leftrightarrow \forall k\langle m\rangle Q_{k}^{n}(\varphi)
$$

ДОКАЗАТЕЛЬСТВо. В $\mathscr{M}_{\mathrm{EA}}^{\infty}$, по свойству редукщии,

$$
\{\langle n+1\rangle \varphi\} \equiv_{n}\left\{Q_{k}^{n}(\varphi): k<\omega\right\}
$$

и эта эквивалентность установлена средствами $\mathrm{EA}^{+}$. Поэтому из $\langle n+1\rangle \varphi$ выводимо некоторое $\Pi_{m+1}$-предложение, если и только если оно следует из $Q_{k}^{n}(\varphi)$ для некоторого $k$. Тем самьм утверждения о $\Pi_{m+1}$-корректности левой и правой части равносильны. 
5.5. Замкнутые формулы GLP и система ординальных обозначений для ординала $\varepsilon_{0}$. Совокупность замкнутых формул логики GLP (по модулю доказуемой эквивалентности) можно также описать как свободную 0-порожденную алгебру Джапаридзе $\mathscr{M}_{0}^{\infty}$, т.е. свободную алгебру, порожденную из констант $\top$ и $\perp$ операциями $\wedge, \neg$ и $\langle n\rangle$ для всех $n$ и удовлетворяюшую тождествам GLP. Заметим, что по теореме Джапаридзе $\mathscr{M}_{0}^{\infty}$ естественным образом вкладьвается в $\mathscr{M}_{T}^{\infty}$, если теория $T$ корректна, и является ее наименьшей подалгеброй ${ }^{6}$ :

$$
\mathscr{M}_{0}^{\infty} \vDash \varphi=\psi \Longleftrightarrow \mathscr{M}_{T}^{\infty} \vDash(\varphi)_{T}^{*}=(\psi)_{T}^{*} .
$$

Строение алгебры $\mathscr{M}_{0}^{\infty}$ было подробно изучено К.Н. Игнатьевым [41], которьй получил теорему о нормальной форме для замкнутого фрагмента GLP и элементарную разрешающую процедуру для этого фрагмента. Мы приводим упрощенньй и несколько ослабленньй вариант этой теоремы.

Замкнутые формулы GLP играют центральную роль в ординальном анализе арифметики Пеано, поскольку их можно рассматривать как конечные представления ординалов до $\varepsilon_{0}$.

Напомним, что ординал $\varepsilon_{0}$ определяется как $\sup \left\{\omega, \omega^{\omega}, \omega^{\omega^{\omega}}, \ldots\right\}$, где $\omega-$ порядковьй тип натуральных чисел. По теореме о канторовской нормальной форме всякий ординал $\alpha<\varepsilon_{0}, \alpha \neq 0$, однозначно представим в виде $\alpha=\omega^{\alpha_{1}}+\cdots+\omega^{\alpha_{n}}$, где $\alpha>\alpha_{1} \geqslant \cdots \geqslant \alpha_{n}$. Раскладывая аналогично показатели $\alpha_{1}, \ldots, \alpha_{n}$ и возникаюшие при этом меньшие показатели, получаем, что всякий ординал $\alpha<\varepsilon_{0}$ представляется в виде замкнутого терма, построенного из константы 0 и функций + и $\omega^{x}$. Мы называем такой терм представлением $\alpha$. Говорим, что представление $\alpha=\omega^{\alpha_{1}}+\cdots+\omega^{\alpha_{n}} \boldsymbol{\theta}$ нормальной форме, если $\alpha_{1} \geqslant \cdots \geqslant \alpha_{n}$ и все подтермы $\alpha_{i}$ также в нормальной форме. Представление ординала $\alpha<\varepsilon_{0}$ в нормальной форме единственно.

Хорошо известно, что отношение быть представлением в нормальной форме и отношение порядка $\alpha<\beta$, как отношения на гёделевых номерах термов, являются элементарными и выразимыми в ЕА. Структуру

$$
\left(\varepsilon_{0} ; 0,<,+, \omega^{x}\right)
$$

вместе с описанной вьше нумерацией назьвают стандартной системой ординальных обозначений до $\varepsilon_{0}$.

Пусть $S$ означает множество полимодальных формул, порожденных из Т операциями $\langle 0\rangle,\langle 1\rangle,\langle 2\rangle, \ldots$ Э Элементы $S$ имеют вид

$$
\alpha=\left\langle n_{1}\right\rangle\left\langle n_{2}\right\rangle \ldots\left\langle n_{k}\right\rangle \top .
$$

Мы отождествляем такие формулы со словами в алфавите $\mathbb{N}$ :

$$
\alpha=n_{1} n_{2} \ldots n_{k} .
$$

Пустое слово $\Lambda$ отождествляем с Т. $S_{n}$ означает ограничение $S$ на алфавит $\{n$, $n+1, \ldots\}$.

\footnotetext{
${ }^{6}$ Если теория $T$ не является корректной, то это уже не верно. В этом случае для некоторого $n$ формула $[n]_{T} \perp$ будет истинна, а значит, $[n]_{T}[n]_{T} \perp$ доказуема в ЕА, т.е. $\mathscr{M}_{T}^{\infty} \vDash[n]_{T}[n]_{T} \perp=\top$.
} 
Для каждого $n$ определим порядок $<_{n}$ на $S_{n}$ как

$$
\beta<_{n} \alpha \Longleftrightarrow \mathbf{G L P} \vdash \alpha \rightarrow\langle n\rangle \beta .
$$

Нетрудно видеть, что $<_{n}$ представляет собой транзитивное отношение. Это отношение также иррефилексивно, поскольку вьводимость $\mathbf{G L P} \vdash \alpha \rightarrow\langle n\rangle \alpha$ влечет $\mathbf{G L P} \vdash$ $\neg \alpha$ по аксиоме Лёба. Но тогда ариф̆метическая интерпретация $(\alpha)_{\text {EA }}^{*}$ опровержима в ЕА и потому ложна, что противоречит корректности ЕА.

Это рассуждение опирается на сильное предположение о корректности ЕА, что допустимо в РА, но не в самой ЕА. Формализуемое в ЕА доказательство иррефлексивности $<_{n}$ можно дать, опираясь на развитую Игнатьевым технику моделей Крипке для GLP, однако здесь нам оно не потребуется. Это же относится и к следующему предложению, установленному в [21].

ПРЕДЛОЖЕНИЕ 5.6. Порядок $\left(S_{n},<_{n}\right)$ фундирован и имеет высоту $\varepsilon_{0}$. По модулю доказуемой эквивалентности в GLP порядок линеен.

Поскольку замкнутый фрагмент логики GLP имеет элементарную разрешаюшую процедуру, отношение порядка $\left(S,<_{0}\right)$ также элементарно. Таким образом, можно рассматривать элементы $S$ как альтернативные представления ординалов до $\varepsilon_{0}$. Укажем простое соответствие между $S$ и ординалами до $\varepsilon_{0}$.

ОПРЕДЕЛЕНИЕ 3 . Положим $o\left(0^{k}\right)=k$. Если $\alpha=\alpha_{1} 0 \alpha_{2} 0 \ldots \alpha_{n-1} 0 \alpha_{n}$, где все $\alpha_{i} \in S_{1}$ и не все из них пусты, то положим

$$
o(\alpha)=\omega^{o\left(\alpha_{n}^{-}\right)}+\cdots+\omega^{o\left(\alpha_{1}^{-}\right)} .
$$

Здесь $\beta^{-}$получается из $\beta \in S_{1}$ заменой каждой буквы $m+1$ на $m$.

Аналогично, $\gamma^{+}$будет означать результат замены в слове $\gamma$ каждой буквы $m$ на $m+1$. Заметим, что $o\left(\gamma^{+}\right)=\omega^{o(\gamma)}$ и отображение $o: S \rightarrow \varepsilon_{0}$, очевидно, сюръективно. Заметим также, что ординал $o(\alpha)$ предельньй, если и только если $\alpha$ начинается с $m>0$.

Предложение 5.6 может быть более явно сформулировано (дл случая $n=0)$ следуюшим образом: для любых $\alpha, \beta \in S$,

$$
\begin{aligned}
& \mathbf{G L P} \vdash \alpha \leftrightarrow \beta \quad \Longleftrightarrow o(\alpha)=o(\beta) ; \\
& \mathbf{G L P} \vdash \beta \rightarrow \diamond \alpha \Longleftrightarrow o(\alpha)<o(\beta) .
\end{aligned}
$$

В качестве примера возьмем слово 2101. Тогда

$$
o(2101)=\omega^{o(0)}+\omega^{o(10)}=\omega+\omega^{\omega^{0}+\omega^{1}}=\omega^{\omega}=o(2) .
$$

Соответственно, в GLP выводимы эквивалентности

$$
\mathbf{G L P} \vdash 2101 \leftrightarrow(21 \wedge 01) \leftrightarrow 21 \leftrightarrow 2 .
$$

Первая из этих эквивалентностей следует из доказьваемой ниже леммы 5.8 (iv).

Отметим без доказательства следуюший вариант теоремы Игнатьева о нормальной форме (см. [41], [21]). 
ПРЕДЛОЖЕНИЕ 5.7. Всякая замкнутая формула әквивалентна в GLP булевой комбиначии формул из $S$.

5.6. Доказательство непротиворечивости РА в стиле Генцена. Здесь мы получим доказательство непротиворечивости РА, опирающееся, помимо узко финитных средств арифметики $\mathrm{EA}^{+}$, лишь на трансфинитную индукцию по ординалу $\varepsilon_{0}$. Первое доказательство такого рода было получено Г. Генценом в знаменитых рабо$\operatorname{Tax}[34],[35]$.

Центральную роль в нашем доказательстве играет свойство редукции градуированных алгебр доказуемости (предложение 5.4). Сначала мы установим, что по модулю вьводимости в GLP множество $S$ замкнуто относительно операции $\langle n+1\rangle \alpha \longmapsto$ $Q_{k}^{n}(\alpha)$.

ЛЕмма 5.8. Следующие выводимости имеют место в GLP:

(i) ecлu $m \leqslant n, m o \vdash n m \alpha \rightarrow m \alpha$;

(ii) $e c \bumpeq u<n<$, mo $\vdash\langle n\rangle \varphi \wedge\langle m\rangle \psi \leftrightarrow\langle n\rangle(\varphi \wedge\langle m\rangle \psi)$;

(iii) $e c л u \alpha \in S_{n+1}, m o \vdash \alpha \gamma \wedge n \beta \leftrightarrow \alpha(\gamma \wedge n \beta)$;

(iv) ecлu $\alpha \in S_{n+1}, m o \vdash \alpha \wedge n \beta \leftrightarrow \alpha n \beta$.

ДокАЗАТЕльство. Утверждение (i) следует из J2 и аксиомы $[m] \varphi \rightarrow[m][m] \varphi$ логики $\mathbf{G L}^{m}$. Утверждение (ii):

$$
\begin{aligned}
\mathbf{G L P} \vdash\langle n\rangle \varphi \wedge\langle m\rangle \psi & \rightarrow[n]\langle m\rangle \psi \quad \text { (по аксиоме J3) } \\
& \rightarrow\langle n\rangle(\varphi \wedge\langle m\rangle \psi) .
\end{aligned}
$$

Обратная импликация следует из (i). Утверждение (iii) получается итерацией (ii), a (iv) вытекает из (iii) для $\gamma=\Lambda$.

ЛЕММА 5.9.

(i) Ecли $\alpha=\langle n+1\rangle \beta$ u $\beta \in S_{n+1}$, то для любого $k$ GLP $\vdash Q_{k}^{n}(\beta) \leftrightarrow(n \beta)^{k+1}$.

(ii) Ecли $\alpha=\langle n+1\rangle \beta$, где $\beta=\gamma m \delta, \gamma \in S_{n+1} u m \leqslant n$, то для любого $k$ $\mathbf{G L P} \vdash Q_{k}^{n}(\beta) \leftrightarrow(n \gamma)^{k+1} m \delta$.

ДокаЗАТЕльство. (i) Рассуждаем индукцией по $k$. Для $k=0$ имеем $Q_{0}^{n}(\beta)=$ $\langle n\rangle \beta \in S$. Шаг индукции:

$$
\begin{aligned}
\operatorname{GLP} \vdash Q_{k+1}^{n}(\beta) & \leftrightarrow\langle n\rangle\left(\beta \wedge(n \beta)^{k+1}\right) \\
& \leftrightarrow\langle n\rangle\left(\beta(n \beta)^{k+1}\right) \quad \text { (по лемме 5.8(iv)) } \\
& \leftrightarrow(n \beta)^{k+2} .
\end{aligned}
$$

(ii) Аналогично, шаг индукции вытекает из

$$
\begin{aligned}
\mathbf{G L P} \vdash Q_{k+1}^{n}(\beta) & \leftrightarrow\langle n\rangle\left(\gamma m \delta \wedge(n \gamma)^{k+1} m \delta\right) \\
& \leftrightarrow\langle n\rangle\left(\gamma\left(m \delta \wedge(n \gamma)^{k+1} m \delta\right)\right) \quad \text { (по лемме 5.8(iii)) } \\
& \leftrightarrow(n \gamma)^{k+2} m \delta \quad \text { (по лемме 5.8(i)). }
\end{aligned}
$$


ОПРЕДЕЛЕНИЕ 4. Для непустого $\alpha \in S$ и $k \in \mathbb{N}$ определим $\alpha \llbracket k \rrbracket$ следующим образом:

- если $\alpha=0 \beta$, то $\alpha \llbracket k \rrbracket=\beta$;

- если $\alpha=\langle n+1\rangle \beta$, то $\alpha \llbracket k \rrbracket$ определено как выражение для $Q_{k}^{n}(\beta)$ из предыдущей леммы.

СлЕДСТВИЕ 5.10. Для любого $k$ u непустого $\alpha \in S \quad \alpha \llbracket k \rrbracket<_{0} \alpha$.

ДокАЗАТЕЛЬСТВо. Индукщией по $k$, очевидно, получаем

$$
\mathbf{G L P} \vdash\langle n+1\rangle \beta \rightarrow Q_{k+1}^{n}(\beta) .
$$

Отсюда по лемме 5.9 для $\alpha=\langle n+1\rangle \beta$

$$
\begin{aligned}
\mathbf{G L P} \vdash \alpha & \rightarrow\langle n\rangle \alpha \llbracket k \rrbracket \\
& \rightarrow\langle 0\rangle \alpha \llbracket k \rrbracket .
\end{aligned}
$$

Обозначим через $\mathrm{TI}^{R}\left(\Pi_{1},<_{0}\right)$ следующее правило вьвода, выражающее трансфинитную индукцию по фундированному порядку $\left(S,<_{0}\right)$ для $\Pi_{1}$-форомул $\varphi$ :

$$
\frac{\forall \alpha \in S\left(\forall \beta<_{0} \alpha \varphi(\beta) \rightarrow \varphi(\alpha)\right)}{\forall \alpha \in S \varphi(\alpha)} .
$$

Мы покажем, что непротиворечивость РА может быть доказана с помошью одного применения этого правила над $\mathrm{EA}^{+}$.

TeOpema 13. $\left[\mathrm{EA}^{+}, \mathrm{TI}^{R}\left(\Pi_{1},<_{0}\right)\right] \vdash \operatorname{Con}(\mathrm{PA})$.

ДокАЗАТЕЛЬСТВо. Мы будем работать в $\mathscr{M}_{\mathrm{EA}}^{\infty}$. Обозначим через $\alpha^{*}$ арифметическую интерпретацию $(\alpha)_{\mathrm{EA}}^{*}$ формулы $\alpha \in S$. Функция $(\cdot)^{*}$ как отображение между гёделевыми номерами формул является элементарной и поэтому определима как терм в ЕА. Мы также пишем $\diamond$ вместо $\diamond_{\text {ЕА. }}$

Вспомним, что по следствию 4.3

$$
\mathrm{PA} \subseteq \mathrm{EA}+\{\langle n\rangle \top: n<\omega\}
$$

Доказательство этого результата, т.е. вьвод аксиомы индукции из соответствующего частного случая равномерной схемы рефлексии, легко формализуемо в ЕА, поэтому мы имеем

$$
\mathrm{EA} \vdash \forall n \diamond(\langle n\rangle \top)^{*} \rightarrow \operatorname{Con}(\mathrm{PA})
$$

Мы докажем $\forall \alpha \in S \diamond \alpha^{*}$ с помощью трансфинитной индукщии над $\mathrm{EA}^{+}$, при этом $\Pi_{1}$-формула $\varphi(\alpha)=\diamond \alpha^{*}$ с переменной $\alpha$ играет роль индукционной формулы. Мы утверждаем, что

$$
\mathrm{EA}^{+} \vdash \forall \alpha \in S\left(\forall \beta<_{0} \alpha \diamond \beta^{*} \rightarrow \diamond \alpha^{*}\right) .
$$

Рассуждая в $\mathrm{EA}^{+}$, допустим, что $\forall \beta<_{0} \alpha \diamond \beta^{*}$. Рассмотрим два случая.

- Если $\alpha=0 \beta$, то $\diamond \beta^{*}$, отсюда $\diamond \diamond \beta^{*}$, опираясь на $\mathrm{RFN}_{\Sigma_{1}}$ (EA) в $\mathrm{EA}^{+}$.

- Если $\alpha=\langle n+1\rangle \beta$, то $\forall k \diamond \alpha \llbracket k \rrbracket^{*}$, поскольку $\alpha \llbracket k \rrbracket<_{0} \alpha$. Заметим, что по следствию 5.5

$$
\mathrm{EA}^{+} \vdash \diamond \alpha^{*} \leftrightarrow \forall k \diamond \alpha \llbracket k \rrbracket^{*}
$$

Поэтому $\forall k \diamond \alpha \llbracket k \rrbracket^{*}$ влечет $\diamond \alpha^{*}$. 
Таким образом,

$$
\begin{aligned}
{\left[\mathrm{EA}^{+}, \mathrm{Tl}^{R}\left(\Pi_{1},<_{0}\right)\right] } & \vdash \forall \alpha \in S \diamond \alpha^{*} \\
& \vdash \operatorname{Con}(\mathrm{PA}) \quad \text { (в силу }(5.1)),
\end{aligned}
$$

что и требовалось доказать.

Укажем теперь, как вывести из теоремы 13 тот же результат для стандартных обозначений ординалов до $\varepsilon_{0}$, основанных на канторовской нормальной форме.

Определим отношение $<_{0}^{\prime}$ на $S$, полагая

$$
\alpha<_{0}^{\prime} \beta \Longleftrightarrow o(\alpha)<o(\beta)
$$

где функция $о$ дана в определении 3. Как нам уже известно из предложения 5.6, на самом деле $<_{0}^{\prime}$ совпадает с $<_{0}$. Однако доказательство этого факта средствами ЕА, которое можно дать с помощью моделей Крипке для замкнутого фрагмента GLP, достаточно сложно. Чтобы избежать этого, мы используем формализацию отношени $<_{0}^{\prime}$ в EA, фундированность которого непосредственно следует из фундированности ординала $\varepsilon_{0}$.

ЛЕМма 5.11. $\mathrm{TI}^{R}\left(\Pi_{1},<_{0}^{\prime}\right)$ сводится $\kappa \mathrm{TI}^{R}\left(\Pi_{1}, \varepsilon_{0}\right)$.

ДоКАЗАТЕльство. Заметим, что функция $о$, как отображение гёделевых номеров слов из $S$ в стандартные представления ординалов до $\varepsilon_{0}$, элементарна. Поэтому индукция для формулы $\varphi(\alpha)$ по $\left(S,<_{0}^{\prime}\right)$ сводится к индукции для формулы

$$
\psi(x):=\forall \alpha \in S(o(\alpha)=x \rightarrow \varphi(\alpha))
$$

по ординалам $x<\varepsilon_{0}$.

ЛЕмма 5.12. Пусть $\alpha=\alpha_{0}^{+} 0 \ldots \alpha_{k-1}^{+} 0 \alpha_{k}^{+}$и о $(\alpha)-$ предельный ординал. Тогда $o(\alpha \llbracket n \rrbracket)= \begin{cases}\omega^{o\left(\alpha_{k}\right)}+\cdots+\omega^{o\left(\alpha_{1}\right)}+\omega^{o(\beta)} \cdot(n+1)+1, & \text { если } о\left(\alpha_{0}\right)=o(\beta)+1 ; \\ \omega^{o\left(\alpha_{k}\right)}+\cdots+\omega^{o\left(\alpha_{1}\right)}+\omega^{o\left(\alpha_{0}[n]\right)}, & \text { если о }\left(\alpha_{0}\right) \text { предельньй. }\end{cases}$

ДокАЗАТЕльство. Поскольку $о(\alpha)$ предельньй, то $\alpha_{0}$ непусто, т.е. $\alpha_{0}^{+}=\langle m+1\rangle \beta$ для некоторого $\beta \in S_{1}$. Слово $\alpha \llbracket n \rrbracket$ определено в соответствии с одним из следующих двух случаев.

(a) $m=0$. Тогда $\alpha \llbracket n \rrbracket=(0 \beta)^{n+1} 0 \alpha_{1}^{+} 0 \ldots \alpha_{k-1}^{+} 0 \alpha_{k}^{+}$, поскольку $\beta \in S_{1}$ и вслед за $\beta$ идет 0.

(b) $m>0$. Тогда $\alpha \llbracket n \rrbracket=\alpha_{0}^{\prime} 0 \alpha_{1}^{+} 0 \ldots \alpha_{k-1}^{+} 0 \alpha_{k}^{+}$, где $\alpha_{0}^{\prime}=\alpha_{0}^{+} \llbracket n \rrbracket=\left(\alpha_{0} \llbracket n \rrbracket\right)^{+}$.

Отсюда следует утверждение леммы.

СЛЕДСТВИЕ 5.13. Для любого $\alpha \neq \Lambda$ и любого $k \quad \alpha \llbracket k \rrbracket<_{0}^{\prime} \alpha$.

Теперь мы можем повторить доказательство теоремы 13 и вьвести

$$
\mathrm{EA}^{+} \vdash \forall \alpha\left(\forall \beta<_{0}^{\prime} \alpha \diamond \beta^{*} \rightarrow \diamond \alpha^{*}\right) \text {. }
$$

Отсюда $\operatorname{Con}(\mathrm{PA})$ следует по правилу $\mathrm{TI}^{R}\left(\Pi_{1},<_{0}^{\prime}\right)$, и мы получаем следуюшую теореmy. 
Teopema 14. $\left[\mathrm{EA}^{+}, \mathrm{Tl}^{R}\left(\Pi_{1}, \varepsilon_{0}\right)\right] \vdash \operatorname{Con}(\mathrm{PA})$.

ЗАмечАниЕ 5.14. Скажем, что $\alpha \in S$ в нормальной форме, если таковопредставление ординала $o(\alpha)$, т.е. если $\alpha=\alpha_{0}^{+} 0 \ldots \alpha_{k-1}^{+} 0 \alpha_{k}^{+}$, где все $\alpha_{i}$ в нормальной форме и $o\left(\alpha_{k}\right) \geqslant \cdots \geqslant o\left(\alpha_{0}\right)$. Слова в нормальной форме представляют собой однозначную систему обозначений для $\varepsilon_{0}$. Для таких слов последовательность $o(\alpha \llbracket n \rrbracket)$ отличается от "стандартной” фундаментальной последовательности для $o(\alpha)$ лишь дополнительным членом “+1" в выражении из леммы 5.12. Доказательство теоремы 14 проходит без изменений и для слов в нормальной форме.

Теоремы 13 и 14 имеют некоторые интересные уточнения. Прежде всего отметим, что правило $\mathrm{Tl}^{R}\left(\Pi_{1}, \varepsilon_{0}\right)$ сводится к формально более слабому правилу трансфинитной индукции для элементарных формул. В самом деле, если данная П 1 -формула имеет вид $\varphi(\alpha)=\forall x \varphi_{0}(\alpha, x)$, то достаточно определить элементарную формулу $\psi(\beta)$ таким образом, чтобы

$$
\psi(\omega \alpha+n) \leftrightarrow \varphi_{0}(\alpha, n)
$$

для любых $\alpha<\varepsilon_{0}$ и $n<\omega$. Тогда индукция для $\varphi$ сводится к индукции для $\psi$.

Кроме того, формула Con(PA) является самой сильной формулой, которую можно вывести из $\mathrm{EA}^{+}$с помошью одного применени правила $\mathrm{TI}^{R}\left(\Pi_{1}, \varepsilon_{0}\right)$.

ПРЕДЛОЖЕниЕ 5.15. $\left[\mathrm{EA}^{+}, \mathrm{Tl}^{R}\left(\Pi_{1}, \varepsilon_{0}\right)\right] \equiv \mathrm{EA}^{+}+\mathrm{Con}(\mathrm{PA})$.

Короткое доказательство можно найти в [21].

Рассмотрим теперь теорию $T=\mathrm{EA}+\mathrm{Con}(\mathrm{PA})$ и соответствуюшую алгебру. Заметим, что $T$ имеет $\Pi_{1}$-аксиоматизацию, поэтому $\mathscr{M}_{T}^{\infty}$ обладает свойством редукции. Нетрудно видеть также, что

$$
\begin{aligned}
\mathrm{EA} \vdash\langle n\rangle_{T} \top & \leftrightarrow\langle n\rangle_{\mathrm{EA}} \operatorname{Con}(\mathrm{PA}) \\
& \leftrightarrow \operatorname{Con}(\mathrm{PA}) \wedge\langle n\rangle_{\mathrm{EA}} \top .
\end{aligned}
$$

Отсюда

$$
T+\mathrm{PA} \equiv T+\left\{\langle n\rangle_{T} \top: n<\omega\right\} .
$$

Таким образом, рассуждение теоремы 13 для алгебры $\mathscr{M}_{T}^{\infty}$ дает следуюший результат:

$$
\left[\mathrm{EA}^{+}+T, \mathrm{Tl}^{R}\left(\Pi_{1}, \varepsilon_{0}\right)\right] \equiv \mathrm{EA}^{+}+\mathrm{Con}(T+\mathrm{PA}) .
$$

Итерируя это рассуждение, получаем характеризацию замькания $\mathrm{EA}^{+}$относительно правила $\mathrm{TI}^{R}\left(\Pi_{1}, \varepsilon_{0}\right)$.

ПРЕДЛОЖЕНИЕ 5.16. $\mathrm{EA}^{+}+\mathrm{Tl}^{R}\left(\Pi_{1}, \varepsilon_{0}\right)$ эквивалентно теории

$$
\mathrm{EA}^{+}+\operatorname{Con}(\mathrm{PA})+\operatorname{Con}(\mathrm{PA}+\operatorname{Con}(\mathrm{PA}))+\cdots .
$$

Аналогично получается и характеризация правила трансфинитной индукции для $\Pi_{n+1}$-формул. При этом возникают итерированные формулы $n$-непротиворечивости PA. Если же рассмотреть правило трансфинитной индукции для произвольных арифметических формул, то это правило будет эквивалентно полной схеме трансфинитной индукции:

$$
\mathrm{TI}\left(\varepsilon_{0}\right): \quad \forall \alpha(\forall \beta<\alpha \varphi(\beta) \rightarrow \varphi(\alpha)) \rightarrow \forall \alpha \varphi(\alpha) .
$$

Отсюда вытекает следуюший результат, полученньй Г. Крайзелем и А. Леви [50] на основе техники Генцена. 
TeOPema 15. $\mathrm{EA}+\mathrm{TI}\left(\varepsilon_{0}\right) \equiv \mathrm{EA}+\mathrm{RFN}(\mathrm{PA}) \equiv \mathrm{PA}+\mathrm{RFN}(\mathrm{PA})$.

ДокАЗАТЕЛЬСтво. Достаточно заметить, что итерированные формулы $n$-непротиворечивости над PA (для любого $n$ ) выводимы из схемы RFN(PA).

5.7. Невыводимое в РА комбинаторное утверждение. Теперь мы приведем простой пример (истинного) утверждения комбинаторного характера, недоказуемого в РА и возникающего из алгебр доказуемости. Это утверждение состоит в конечности некоторой последовательности, похожей на известную игру "Геракл и Гидра" Л. Кирби и Дж. Париса [46].

В игре участвуют слова в алфавите $\mathbb{N}$, которые мы будем по аналогии с Гидрой называть червями. Последний элемент червя $w=x_{0} x_{1} \ldots x_{n}$ назьвается головой.

Игра начинается с произвольного червя и неформально может быть описана следуюшим образом. На очередном шаге Геракл наносит червю удар, в результате чего голова червя уменьшается на 1 . В ответ червь регенерируется в соответствии с двумя простьми правилами, описанньми ниже. В отличие от игры "Геракл и Гидра", возникающая последовательность слов полностью детерминирована.

Более формально, мы определим функцию $\operatorname{next}(w, m)$, где $w=x_{0} \ldots x_{n}$ - червь, а $m \in \mathbb{N}$ - шаг последовательности.

1. Если $x_{n}=0$, то $\operatorname{next}(w, m):=x_{0} \ldots x_{n-1}$. В этом случае голова червя отрубается.

2. Если $x_{n}>0$, положим $k:=\max \left\{i<n: x_{i}<x_{n}\right\}$.

Червь $w$ (с головой, уменьшенной на 1$)$ состоит из двух частей: здоровой ${ }^{7} r:=$ $x_{0} \ldots x_{k}$ и больной $s:=x_{k+1} \ldots x_{n-1}\left(x_{n}-1\right)$. Полагаем

$$
\operatorname{next}(w, m):=r * \underbrace{s * s * \cdots * s}_{m+1 \text { раз }} .
$$

Последовательность червей, начинаюшаяся с произвольного червя $w$, определяется следуюшим образом:

$$
w_{0}:=w \quad \text { и } \quad w_{n+1}:=\operatorname{next}\left(w_{n}, n+1\right) .
$$

Рассмотрим, к примеру, червя $w=2031$. На первом шаге получаем $k=1 ; r=20$; $s=30 ; \operatorname{next}(w, 1)=203030$. Возникает последовательность:

$$
\begin{aligned}
& w_{0}=2031 \\
& w_{1}=203030 \\
& w_{2}=20303 \\
& w_{3}=20302222, \\
& w_{4}=203022212221222122212221, \\
& w_{5}=2030(22212221222122212220)^{6},
\end{aligned}
$$

7 Эта часть может быть и пустой. 
Заметим, что последовательность $w_{n}$ определена примитивной рекурсией. Фактически, $w_{n}$ есть элементарная функция $n$ и (кода) $w$. Это вытекает из простой оценки

$$
\left|w_{n}\right| \leqslant(n+2) !\left|w_{0}\right|,
$$

показываюшей, что длина червей последовательности растет лишь мультиэкспоненциально. Заметим также, что максимальньй элемент червя может лишш уменьшаться. Это позволяет вьписать элементарную формулу с тремя переменными, выражающую $w_{n}=u$.

Интересующее нас утверждение WP говорит о том, что рано или поздно Геракл победит любого червя: $\forall w \exists n w_{n}=\Lambda$.

Tеорема 16. WP истинно, но не доказуемо в PA. Фактически, WP эквивалентно 1-Con(PA) над EA.

ДокАЗАТЕльСтво. Черви имеют естественную интерпретацию в терминах алгебры доказуемости $\mathscr{M}_{\mathrm{EA}}^{\infty}$. Пусть слово $\alpha \in S$ обратно к $w$, т.е. совпадает с $w$, записанным в обратном порядке. Нетрудно заметить, что тогда червь $\operatorname{next}(w, n)$ будет обратен к слову $\alpha \llbracket n \rrbracket$ (это не случайно, поскольку правила игры были фактически списаны нами с определения $\alpha \llbracket n \rrbracket)$. Отсюда следует истинность WP, поскольку в силу следствия 5.13 последовательность соответствуюших ординалов убывает.

Фактически, это рассуждение показьвает, что WP доказуемо с помощью одного применения правила $\mathrm{TI}^{R}\left(\Pi_{2}, \varepsilon_{0}\right)$. Отсюда следует вьводимость WP из 1-Con (PA) в силу аналогапредложения 5.15. Однако здесь мы докажем утверждение EA + 1-Con(PA) $\vdash$ WP более прямьм (и более интересным) методом, не используюшим ординалы.

Определим арифметическую интерпретацию $w^{\star}:=\left(\alpha^{+}\right)_{\text {EA }}^{*}$, где $\alpha$ обратно к $w$. Как

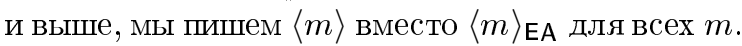

Лемма 5.17. Для любого $w$ PA $\vdash w^{\star}$.

ДоКАЗАТЕЛЬСТвО. Индукция по $|w|$. Если $w=v n$ и $m$ больше любой буквы $w$, то

$$
\begin{aligned}
\mathrm{EA} \vdash v^{\star} \wedge\langle m+1\rangle \top & \rightarrow\langle m+1\rangle v^{\star} \text { по лемме } 5.8 \\
& \rightarrow\langle n+1\rangle v^{\star} .
\end{aligned}
$$

По следствию 4.3 и предположению индукции

$$
\mathrm{PA} \vdash v^{\star} \wedge\langle m+1\rangle \top ;
$$

таким образом, $\mathrm{PA} \vdash\langle n+1\rangle v^{\star}$, откуда следует шаг индукции.

Лемма 5.18. Для любого $\mathrm{w}$

$$
\mathrm{EA} \vdash \forall n\left(w_{n} \neq \Lambda \rightarrow \square\left(w_{n}^{\star} \rightarrow\langle 1\rangle w_{n+1}^{\star}\right)\right) .
$$


ДокАЗАТЕльСТво. Достаточно доказать в ЕА

$$
\forall w \neq \Lambda \forall n \mathrm{EA} \vdash w^{\star} \rightarrow\langle 1\rangle \operatorname{next}(w, n)^{\star} .
$$

Пусть $\alpha$ обратно к $w$, тогда в силу сделанного замечания $\alpha \llbracket n \rrbracket$ обратно к $\operatorname{next}(w, n)$. По следствию 5.10 (формализуемому в ЕА)

$$
\mathbf{G L P} \vdash \alpha \rightarrow \diamond \alpha \llbracket n \rrbracket .
$$

Теоремы GLP переходят в себя при отображении $(\cdot)^{+}$, поэтому

$$
\mathbf{G L P} \vdash \alpha^{+} \rightarrow\langle 1\rangle \alpha \llbracket n \rrbracket^{+} .
$$

Отсюда вытекает требуемое в силу ариф̆метической корректности GLP.

ЛЕмма 5.19. Для любого $\mathrm{w}$

$$
\mathrm{EA} \vdash\langle 1\rangle w_{0}^{\star} \rightarrow \exists n w_{n}=\Lambda .
$$

ДокАЗАТЕльство. Мы докажем $\forall n w_{n} \neq \Lambda \rightarrow \forall n[1] \neg w_{n}^{\star}$, опираясь на теорему Лёба:

$$
\begin{aligned}
\mathrm{EA} \vdash \forall n w_{n} \neq \Lambda \wedge[1] \forall n[1] \neg w_{n}^{\star} & \rightarrow[1] \forall n[1] \neg w_{n+1}^{\star} \\
& \rightarrow \forall n[1][1] \neg w_{n+1}^{\star} \\
& \rightarrow \forall n[1] \neg w_{n}^{\star} \quad \text { по лемме } 5.18 ; \\
\text { EA } \vdash[1] \forall n w_{n} \neq \Lambda & \rightarrow[1]\left([1] \forall n[1] \neg w_{n}^{\star} \rightarrow \forall n[1] \neg w_{n}^{\star}\right) \\
& \rightarrow[1] \forall n[1] \neg w_{n}^{\star} \quad \text { по теореме Лёба; } \\
\text { ЕА } \vdash \forall n w_{n} \neq \Lambda & \rightarrow[1] \forall n w_{n} \neq \Lambda \quad\left(\Sigma_{2} \text {-полнота }\right) \\
& \rightarrow[1] \forall n[1] \neg w_{n}^{\star} \\
& \rightarrow \forall n[1] \neg w_{n}^{\star} \\
& \rightarrow[1] \neg w_{0}^{\star},
\end{aligned}
$$

что и требовалось доказать.

Мы завершаем доказательство первой части теоремы 16 . По леммам 5.17 и 5.19 получаем, что для любого $w$

$$
\begin{aligned}
& \mathrm{PA} \vdash\langle 1\rangle w^{\star}, \\
& \mathrm{EA} \vdash\langle 1\rangle w^{\star} \rightarrow \exists n w_{n}=\Lambda .
\end{aligned}
$$

Значит, $\forall w$ PA $\vdash \exists n w_{n}=\Lambda$. Это доказательство формализуемо в ЕА, поэтому 1-Con(РA) влечет $\forall w \exists n w_{n}=\Lambda$. 
5.8. Независимость WP. Положим $w \llbracket n \rrbracket:=\operatorname{next}(w, n)$ и

$$
w \llbracket n \ldots n+k \rrbracket:=w \llbracket n \rrbracket \llbracket n+1 \rrbracket \ldots \llbracket n+k \rrbracket .
$$

Мы введем следуюший аналог так называемых функций Харди. Положим $h_{w}(n)$ равным наименьшему $k$ такому, что

$$
w \llbracket n \ldots n+k \rrbracket=\Lambda .
$$

Нам будут нужны некоторые свойства $h$, которые можно установить элементарными рассуждениями, формализуемьми в ЕА. Заметим, что функция

$$
W(w, n, k):=w \llbracket n \ldots n+k \rrbracket
$$

элементарна, поскольку ее можно определить ограниченной рекурсией по $k$ аналогично последовательности $w_{n}$. Это дает естественное элементарное представление отношения $h_{w}(n)=k$ в $\mathrm{EA}$.

Следующее понятие удобно, чтобы установить монотонность функций $h_{w}$. Положим $v \unlhd u$, если и только если $v=u \llbracket 0 \rrbracket \llbracket 0 \rrbracket \ldots \llbracket 0 \rrbracket$. Это означает, что $v$ есть начальный сегмент $u$, за исключением последней буквы, которая не превосходит соответствующей буквы в $u$. Заметим, что в ЕА, вообще говоря, нельзя установить тотальность функций $h_{w}$. Дл формализации нижеследуюших лемм в ЕА мы каждый раз будем оговаривать это условие.

Лемма 5.20. Если $h_{w}(m)$ определено $и$ u $\unlhd w, m o$

$$
\exists k w \llbracket m \ldots m+k \rrbracket=u .
$$

ДокАЗАТЕЛЬСТво. Правила игры таковы, что $n$-я буква $w$ может измениться, если только все буквы справа от нее удалены. Поэтому если $w \llbracket m \ldots m+s \rrbracket=\Lambda$, то для некоторого $k<s w \llbracket m \ldots m+k \rrbracket=u$.

СлЕДСТвиЕ 5.21. Если $h_{w}(n)$ определено, $m o \forall m \leqslant n \exists k w \llbracket n \ldots n+k \rrbracket=w \llbracket m \rrbracket$.

ДокАЗАТЕльство. $w \llbracket m \rrbracket$ есть начало $w \llbracket n \rrbracket$.

Лемма 5.22. Eсли $v \unlhd u$ u $x \leqslant y, m o h_{v}(x) \leqslant h_{u}(y)$.

ДокАЗАТЕльСТво. Применяя следствие 5.21 несколько раз, получаем $s_{0}, s_{1}, \ldots$ такие, что

$$
\begin{aligned}
u \llbracket y \ldots y+s_{0} \rrbracket & =v \llbracket x \rrbracket, \quad \text { где } y+s_{0} \geqslant x ; \\
u \llbracket y \ldots y+s_{0}+s_{1} \rrbracket & =v \llbracket x \rrbracket \llbracket x+1 \rrbracket, \quad \text { где } y+s_{0}+s_{1} \geqslant x+1 ;
\end{aligned}
$$

Поэтому все элементы последовательности, начинаюшейся с $v$, входят в последовательность для $u$.

Лemma 5.23. $h_{u 0 v}(n)=h_{u}\left(n+h_{v}(n)+2\right)+h_{v}(n)+1>h_{u}\left(h_{v}(n)\right)$. 
ДокАЗАТЕльство. Элемент 0 между $u$ и $v$ останется на месте до тех пор, пока часть $v$ не будет удалена. Поэтому червь $u 0 v$ сначала перейдет в $u 0$ за $h_{v}(n)$ шагов, а затем в $\Lambda$.

СлЕДСТВИЕ 5.24. Если $w \in S_{1}, m_{o} h_{w 1}(n)>h_{w}^{(n)}(n)$.

ДокАЗАТЕЛЬСтво. Достаточно заметить, что $w 1 \llbracket n \rrbracket=w 0 w 0 \ldots w 0$.

Теперь мы сформулируем основную лемму. Как обычно, $h_{w} \downarrow$ означает формулу $\forall x \exists y h_{w}(x)=y$. Определим также $w^{*}:=\alpha^{*}$, где слово $\alpha \in S$ обратно к $w$.

ЛЕмма 5.25. EA $\vdash \forall w \in S_{1}\left(h_{1111 w \downarrow} \rightarrow\langle 1\rangle w^{*}\right)$.

Из этой леммы легко следует теорема 16.

ДОКАЗАТЕЛЬСТВО НЕЗАВИСИМОСТИ WP.

$$
\begin{aligned}
\mathrm{EA} \vdash \forall w \exists n w_{n}=\Lambda & \rightarrow \forall w \in S_{1} h_{w} \downarrow \\
& \rightarrow \forall n\langle 1\rangle\langle n\rangle \top \\
& \rightarrow 1-\operatorname{Con}(\mathrm{PA}) .
\end{aligned}
$$

Здесь первая импликация имеет место, поскольку для любого червя $w$ и числа $x$ мы можем найти $w^{\prime}:=w 0^{x}$ такой, что $w^{\prime} \llbracket 0 \ldots x-1 \rrbracket=w$. Следовательно, Геракл побеждает $w^{\prime}$, если и только если значение $h_{w}(x)$ определено.

В доказательстве леммы мы существенно опираемся на лемму 3.5. Заметим, что по следствию 5.24 мы имеем $h_{111}(x)>2^{x}$, поэтому то же неравенство имеет место и для функции $h_{111 w}(x)$, где $w$ - любое слово.

ДокаЗАТЕльство ЛЕммы 5.25. Рассуждаем в ЕА. По теореме Лёба мы можем использовать, как дополнительное, предположение

$$
\forall w \in S_{1}[1]\left(h_{1111 w \downarrow} \rightarrow\langle 1\rangle w^{*}\right) .
$$

Если $1111 w=v 1$, то $h_{v 1} \downarrow \rightarrow \lambda x \cdot h_{v}^{(x)}(x) \downarrow$.

Функция $h_{v}$ монотонно возрастает, имеет элементарный график и растет быстрее $2^{x}$. Поэтому для $w=\Lambda$ утверждение очевидно: $h_{1111} \downarrow$ влечет тотальность $2_{n}^{x}$ и, следовательно, $\langle 1\rangle \top$. Если $w$ непусто, мы рассуждаем следующим образом:

$$
\begin{aligned}
\lambda x . h_{v}^{(x)}(x) \downarrow & \rightarrow\langle 1\rangle h_{v} \downarrow \\
& \rightarrow\langle 1\rangle\langle 1\rangle v^{*} \quad \text { (по предположению (5.2)) } \\
& \rightarrow\langle 1\rangle w^{*} .
\end{aligned}
$$

Если $1111 w=v$ оканчивается на $m>1$, то

$$
\begin{aligned}
h_{v} \downarrow & \rightarrow \lambda x . h_{v \llbracket x \rrbracket}(x+1) \downarrow \\
& \rightarrow \forall n h_{v \llbracket n \rrbracket \downarrow}
\end{aligned}
$$

Для вывода последней импликации рассмотрим произвольное $n$. Если $x \leqslant n$, то $h_{v \llbracket n \rrbracket}(x) \leqslant h_{v \llbracket n \rrbracket}(n+1)$. Если же $x \geqslant n$, то $h_{v \llbracket n \rrbracket}(x) \leqslant h_{v \llbracket x \rrbracket}(x+1)$. В обоих случаях большее значение определено, значит, определено и меньшее. 
Продолжим рассуждение:

$$
\begin{aligned}
& \left.\forall n h_{v \llbracket n+1 \rrbracket} \rightarrow \forall n h_{v \llbracket n \rrbracket 1 \downarrow} \quad \text { (поскольку } v \llbracket n \rrbracket 1 \unlhd v \llbracket n+1 \rrbracket\right) \\
& \rightarrow \forall n\langle 1\rangle h_{v \llbracket n \rrbracket} \quad \text { (как и ранее) } \\
& \rightarrow \forall n\langle 1\rangle\langle 1\rangle w \llbracket n \rrbracket^{*} \\
& \rightarrow\langle 1\rangle w^{*} \text { (по свойству редукции). }
\end{aligned}
$$

Это завершает доказательство леммы 5.25 и теоремы 16.

\section{§ 6. Итерированные схемы рефлексии}

Здесь мы коротко рассмотрим связь алгебр доказуемости и иерархий итерированных схем реффлексии в смысле Тьюринга-Фефермана [92], [32]. Исторически первьм и, вероятно, наиболее важньм примером такой иерархии является так называемая трансфинитная рекурсивная прогрессия Тьюринга, которая определяется следуюшим образом:

T1. $T_{0}:=T$, где $T$ - данная “начальная" теория;

T2. $T_{\alpha+1}:=T_{\alpha}+\operatorname{Con}\left(T_{\alpha}\right)$;

Т3. $T_{\alpha}:=\bigcup_{\beta<\alpha} T_{\beta}$, где $\alpha-$ предельньй ординал.

По теореме Гёделя если начальная теория $T \Sigma_{1}$-корректна, то теории $T_{\alpha}$ образуют строго возрастающую трансфинитную последовательность $\Sigma_{1}$-корректных расширений $T$. Эта последовательность позволяет сопоставить ординал $\operatorname{ord}_{T}(U)$ любому расширению $U$ теории $T$ следуюшим образом:

$$
\operatorname{ord}_{T}(U):=\text { наименьший } \alpha \text { такой, что } U \nvdash \operatorname{Con}\left(T_{\alpha}\right) \text {. }
$$

Для наиболее естественных расширений теории $T$ обычно можно установить, что для $\alpha=\operatorname{ord}_{T}(U)$ теория $T_{\alpha}$ исчерпывает все арифметические $\Pi_{1}$-следствия $U$, т.е.

$$
U \equiv_{\Pi_{1}} T_{\alpha}
$$

Таким образом, $\operatorname{ord}_{T}(U)$ может рассматриваться как ординал, измеряющий “силу" теории $U$ относительно $T$ в смысле доказуемости П 1 -предложений. В качестве $T$ естественно выбрать некоторую фиксированную слабую П 1 -аксиоматизированную теорию, например ЕА. В этом случае мы будем назьвать $\operatorname{ord}_{\mathrm{EA}}(U) \Pi_{1}^{0}$-ординалом $U$.

Преимущество этого подхода перед другими известньми способами сопоставления ординалов теориям состоит в том, что при этом получается наиболее тонкая классификация, позволяюшая различать теории уже на уровне их $\Pi_{1}$-следствий. Например, теории PA и PA + Con(PA) имеют одни и те же классы доказуемо тотальных вычислимых функций и одинаковые ординальные границы доказуемости трансфинитной индукции, равные $\varepsilon_{0}$. Однако (см. ниже следствия 6.1 и 6.4) $\operatorname{ord}_{\mathrm{EA}}(\mathrm{PA})=\varepsilon_{0}$, в то время как $\operatorname{ord}_{\mathrm{EA}}(\mathrm{PA}+\operatorname{Con}(\mathrm{PA}))=\varepsilon_{0} \cdot 2$. Аналогично, заменяя в определении прогрессии Тьюринга формулу непротиворечивости $\operatorname{Con}(T)$ на формулу $n$-непротиворечивости $n$-Con $(T)$, мы получаем определение $\Pi_{n}^{0}$-ординала теории.

Хорошо известная трудность на пути этой программы классификации теорий состоит в том, что условия Т1-Т3 не определяют последовательность $T_{\alpha}$ однозначно, 
т.е. теория $T_{\alpha}$ зависит от формального представления ординала $\alpha$ в арифметике, а не только от самого порядкового типа, а также зависит от конкретного способа арифметизации условий Т1-Т3.

Для анализа этой проблемы С. Феферман [32] рассмотрел семейство теорий вида $\left(T_{c}\right)_{c \in \mathscr{O}}$, удовлетворяющее условиям Т1-Т3 вдоль каждого пути в $\mathscr{O}$, где $\mathscr{O}-$ универсальна система ординальных обозначений Клини. Используя идею Тьюринга, он показал, что каждое истинное $\Pi_{1}$-предложение доказуемо в $T_{c}$ для подходящего ординального обозначения $c \in \mathscr{O}$, где $|c|=\omega+1 .^{8}$ Отсюда следует, что найдутся два обозначения $a, b \in \mathscr{O},|a|=|b|=\omega+1$, такие, что в $T_{a}$ доказуемо $\operatorname{Con}\left(T_{b}\right)$. Это обстоятельство препятствует реализации программы сопоставления ординалов теориям, описанной выше, по крайней мере в обшем случае.

Тем не менее, остается возможность, что для естественных, математически осмысленных теорий $U$ можно исчерпать все $\Pi_{1}$-следствия $U$, используя лишш специфические, естественные ординальные обозначения, и тщательный выбор таких обозначений должен дать правильные ординальные оценки. Первые примеры успешной реализации этой идеи были получены в работе У. Шмерля [77], которьй показал среди прочего, что для стандартной системы ординальных обозначений до $\varepsilon_{0}$ и некоторого конкретного способа арифметизации T1-T3 в теориях PA и PRA $\varepsilon_{0}$ доказуемы одни и те же П 1 -предложения. Таким образом, $\operatorname{ord}_{\mathrm{PRA}}(\mathrm{PA})=\varepsilon_{0}$, что совпадает с ординалом, сопоставляемьм РА посредством других теоретико-доказательственных методов.

В работе [12] предложен упрощенньй подход к определению итерированных схем рефлексии на основе понятия гладкой прогрессии Тьюринга. Там же установлены теоремы о существовании и единственности таких прогрессий для данного элементарного линейного порядка и данной начальной теории. Тем самьм понятие гладкости дает удовлетворительный ответ на вопрос об арифметизации условий Т1-Т3 и их аналогов для других схем рефлексии. Это позволило дать корректные определения $\Pi_{n}^{0}$-ординалов теорий в соответствии с изложенным вьше подходом для любой фиксированной системы ординальных обозначений [19]. В этой же работе содержится подробное обсуждение возможных определений ординалов теорий и обзор основных результатов об итерированных схемах реф̆лексии.

Здесь мы покажем, что система ординальных обозначений, возникающая из градуированной алгебры доказуемости для ЕА, позволяет непосредственно вычислить $\Pi_{n}^{0}$-ординалы стандартных фрагментов РА для всех $n$. Мы также легко получаем отсюда вариант так назьваемой формулы Шмерля, связьвающей различные прогрессии итерированных схем равномерной реффлексии. Предлагаемый здесь подход является упрощением [19].

6.1. Гладкие прогрессии Тьюринга. Назовем элементарнымм частичныл порядком $(D, \prec)$ пару элементарных формул $x \in D$ и $x \prec y$ таких, что в ЕА доказуемо, что отношение $\prec$ строго частично упорядочивает множество $D \subseteq \mathbb{N}$. Элементарное вполне упорядочение - это элементарньй порядок, являюшийся фундированным порядком в стандартной модели арифметики. (Заметим, что понятие фундированности невыразимо в языке арифметики первого порядка.)

\footnotetext{
${ }^{8}$ Здесь и ниже $|c|$ означает ординал, кодируемшй числом $c$.
} 
Допустим, что нам даны некоторая "начальная" элементарно аксиоматизированная теория $T$ и элементарньй порядок $(D, \prec)$. В [12], [19] показано, что сушествует единственное с точностью до доказуемой эквивалентности в ЕА семейство элементарно аксиоматизированных теорий $\left(T_{\alpha}^{n}\right)_{\alpha \in D}$ такое, что в ЕА доказуемо

$$
\forall \alpha \in D T_{\alpha}^{n} \equiv T+\left\{n-\operatorname{Con}\left(T_{\beta}^{n}\right): \beta \prec \alpha, \beta \in D\right\} .
$$

Такую последовательность будем называть гладкой прогрессией, основанной на итерации формулы п-непротиворечивости. Обозначаем через $T_{\alpha}$ гладкую прогрессию Тьюринга $T_{\alpha}^{0}$. Назовем $\Pi_{n+1}^{0}$-ординалом теории $U$ наименьший $\alpha \in D$, для которого $\mathrm{EA}_{\alpha+1}^{n} \nsubseteq U$.

Рассмотрим $\left(S_{n},<_{n}\right)$ в качестве порядка $(D, \prec)$ и соответствуюшую прогрессию $T_{\alpha}^{n}$.

ТЕОРемА 17. Если $T$ - әлементарно аксиоматизированное $\Pi_{n+1}$-расширение $\mathrm{EA}^{+}$, то доказуемо в $\mathrm{EA}^{+}$

$$
\forall \alpha \in S_{n} T+\alpha_{T}^{*} \equiv{ }_{n} T_{\alpha}^{n}
$$

ДокАЗАТЕльство. По теореме Лёба, достаточно установить

$$
\mathrm{EA}^{+} \vdash \forall \beta<_{n} \alpha \square_{\mathrm{EA}^{+}}\left(T+\beta_{T}^{*} \equiv_{n} T_{\beta}^{n}\right) \rightarrow T+\alpha_{T}^{*} \equiv_{n} T_{\alpha}^{n} .
$$

Мы дадим неформальное доказательство эквивалентности $T+\alpha_{T}^{*} \equiv{ }_{n} T_{\alpha}^{n}$ в $\mathrm{EA}^{+}$, при этом посылку импликации назьваем рефлексивньм предположением индукции. Заметим, что это предположение влечет

$$
\forall \beta<_{n} \alpha \mathrm{EA}^{+} \vdash\langle n\rangle_{T} \beta^{*} \leftrightarrow n-\operatorname{Con}\left(T_{\beta}^{n}\right) .
$$

$(\supseteq)$ По определению $<_{n}$, если $\beta<_{n} \alpha$, то $T \vdash \alpha_{T}^{*} \rightarrow\langle n\rangle_{T} \beta_{T}^{*}$. По рефилексивному предположению индукции

$$
\mathrm{EA}^{+} \vdash\langle n\rangle_{T} \beta_{T}^{*} \rightarrow n-\operatorname{Con}\left(T_{\beta}^{n}\right) .
$$

Отсюда следует, что для всех $\beta<_{n} \alpha$

$$
T \vdash \alpha_{T}^{*} \rightarrow n-\operatorname{Con}\left(T_{\beta}^{n}\right),
$$

т.е. $T+\alpha_{T}^{*} \vdash T_{\alpha}^{n}$.

$\left(\subseteq_{n}\right)$ Допустим $T+\alpha^{*} \vdash \pi$, где $\pi \in \Pi_{n+1}$. Если $\alpha=n \beta$, то $T+\langle n\rangle_{T} \beta_{T}^{*} \vdash \pi$, что влечет $T+n$-Con $\left(T_{\beta}^{n}\right) \vdash \pi$ по рефлексивному предположению индукции. Поскольку $\beta<_{n} \alpha$, заключаем, что $T_{\alpha}^{n} \vdash \pi$.

Если $\alpha=\langle m+1\rangle \beta$, где $m \geqslant n$, то

$$
\left\{\alpha_{T}^{*}\right\} \equiv_{m}\{\alpha \llbracket k \rrbracket: k<\omega\}
$$

по свойству редукции. Поэтому $T+\alpha \llbracket k \rrbracket_{T}^{*} \vdash \pi$ для некоторого $k$. Поскольку $\alpha \llbracket k \rrbracket<_{n}$ $\alpha$, мы получаем $T_{\alpha \llbracket k \rrbracket}^{n} \vdash \pi$ по рефлексивному предположению индукции.

Заметим, что в доказательстве этой теоремы мы нигде не воспользовались свойством фундированности системы ординальных обозначений. Тот факт, что $T$ содержит $\mathrm{EA}^{+}$, сушественен лиш при $n=0$; для $n>0$ достаточно предположения о том, что $T \supseteq$ ЕА. Это относится и к формулируемым ниже следствиям.

Так же как и для теоремы Генцена, мы имеем вариант этого результата для стандартной системы обозначений для $\varepsilon_{0}$. Обозначим через $o_{n}(\alpha)$ канторовскую нормальную форму представления ординала $o\left(\alpha^{-n}\right)$, где $\alpha^{-n}$ получается из $\alpha \in S_{n}$ заменой всех букв $m$ на $m-n$. 
ТЕОРема 18. Если $T$ - элементарно аксиоматизированное $\Pi_{n+1}$-расширение $\mathrm{EA}^{+}$, то доказуемо в $\mathrm{EA}^{+}$

$$
\forall \alpha \in S_{n} \quad T+\alpha_{T}^{*} \equiv_{n} T_{o_{n}(\alpha)}^{n} .
$$

Эта теорема позволяет вычислить $\Pi_{n}^{0}$-ординалы и получить характеризации $\Pi_{n}$-следствий для всех рассматривавшихся ранее фрагментов РА. Для схем индукции с параметрами ответ дает следуюшая теорема. Обозначим $\omega_{0}(\alpha)=\alpha, \omega_{n+1}(\alpha)=$ $\omega^{\omega_{n}(\alpha)}, \omega_{n}=\omega_{n}(1)$. Заметим, что $o(n)=\omega_{n}$.

СЛЕДСТВИЕ 6.1 .

(i) $\mathrm{PA} \equiv m \bigcup_{\alpha<\varepsilon_{0}}\left(\mathrm{EA}^{+}\right)_{\alpha}^{m}$.

(ii) $I \Sigma_{n} \equiv_{m}\left(\mathrm{EA}^{+}\right)_{\omega_{n+1-m}}^{m}$, ecлu $m \leqslant n$.

ДокАЗАТЕльство. Утверждение (ii) следует из того, что $I \Sigma_{n}$ есть $\langle n+1\rangle_{\text {ЕА }} \top$ и $o_{m}(n+1)=o(n+1-m)=\omega_{n+1-m}$. Первое утверждение получается отсюда переходом к пределу по $n$.

Характеризация $\Pi_{2}$-ординала некоторой теории в терминах итерированных формул 1-непротиворечивости над ЕА влечет характеризацию доказуемо тотальных вычислимых функций этой теории в терминах так назьваемой расширенной иерархии Гжегорчика $\mathscr{E} \alpha$, т.е. $\mathscr{F}\left(\mathrm{EA}_{\alpha}^{2}\right)=\mathscr{E}^{\alpha}$ для любых $\alpha$.

Этот результат имеет место для достаточно общих систем ординальных обозначений и доказывается трансфинитной итерацией леммы 3.5 ([19], см. также [89]). Отсюда получается новое доказательство известной теоремы Швихтенберга-Вэйнера (см. $[28],[19])$.

СЛЕДСТВИЕ 6.2. $\mathscr{F}(\mathrm{PA})=\bigcup_{\alpha<\varepsilon_{0}} \mathscr{E}^{\alpha}$.

Рассмотрим теперь более экзотический случай теории $I \Sigma_{1}+I \Pi_{2}^{-}$. Эта теория интересна тем, что в ней формализуемо наиболее естественное доказательство тотальности функции Аккермана. Следующая теорема, в частности, позволяет дать ответ на вопрос о классе доказуемо тотальных вычислимых функций этой теории.

Tеорема 19. $I \Sigma_{n}+I \Pi_{n+1}^{-} \equiv_{m}\left(\mathrm{EA}^{+}\right)_{\alpha}^{m}$, əде $\alpha=\omega_{n+1-m}(2), m \leqslant n$.

ДОКАЗАТЕЛЬСТВО. По предложению $4.23, I \Sigma_{n}+I \Pi_{n+1}^{-} \equiv_{n}\left(I \Sigma_{n}\right)_{\omega}^{n}$. По свойству редукции для $\mathscr{M}_{\mathrm{EA}}^{\infty}{ }^{\infty}$ мы также имеем

$$
\left(I \Sigma_{n}\right)_{\omega}^{n} \equiv_{n}\langle n+1\rangle\left(I \Sigma_{n}\right) \equiv\langle n+1\rangle\langle n+1\rangle \top .
$$

По теореме $18, \Pi_{n}$-ординал этой теории равен

$$
o_{m}(\langle n+1\rangle\langle n+1\rangle \top)=o(\langle n+1-m\rangle\langle n+1-m\rangle \top)=\omega_{n+1-m}(2) .
$$

СлЕДСТВИЕ 6.3. $\mathscr{F}\left(I \Sigma_{n}+I \Pi_{n+1}^{-}\right)=\mathscr{E}_{n}(2) . \quad$ В частности, $\mathscr{F}\left(I \Sigma_{1}+I \Pi_{2}^{-}\right)=$ $\mathscr{E}^{2}$, т.е. совпадает с классом дваждди рекурсивных функиий в смысле Р. Петер.

Наконец, вычислим П 10 -ординал теории PA + Con(PA). Мы будем работать со стандартной системой обозначений до $\varepsilon_{1}$, которая представляет собой структуру $\left(\varepsilon_{1} ; \leqslant\right.$, $\left.0, \varepsilon_{0},+, \omega^{x}\right)$. Ординалы кодируются термами в нормальной форме, построенными из констант 0 и $\varepsilon_{0}$ с помошью функций + и $\omega^{x}$. 
СлЕДСТвиЕ 6.4. $\mathrm{PA}+\operatorname{Con}(\mathrm{PA}) \equiv_{0} \mathrm{EA}_{\varepsilon_{0}+\varepsilon_{0}}^{+}$.

ДокАЗАТЕЛЬСтво. Рассмотрим теорию $T=\mathrm{EA}^{+}+\mathrm{Con}(\mathrm{PA})$ и соответствуюшую алгебру доказуемости $\mathscr{M}_{T}^{\infty}$. Заметим, что $T$ является $\Pi_{2}$-расширением ЕА, поэтому $\mathscr{M}_{T}^{\infty}$ обладает свойством редукции. Как мы уже видели,

$$
\mathrm{PA}+\operatorname{Con}(\mathrm{PA}) \equiv T+\left\{\langle n\rangle_{T} \top: n<\omega\right\} .
$$

Отсюда следует, по теореме 18 , что

$$
\mathrm{PA}+\operatorname{Con}(\mathrm{PA}) \equiv_{0} T_{\varepsilon_{0}} .
$$

$\mathrm{C}$ другой стороны, доказуемо в $\mathrm{EA}^{+}$мы имеем $\mathrm{PA} \equiv_{0} \mathrm{EA}_{\varepsilon_{0}}^{+}$, откуда

$$
T \equiv \mathrm{EA}_{\varepsilon_{0}+1}^{+}
$$

По теореме о единственности гладких прогрессий, если доказуемо $U \equiv V$, то доказуемо $U_{\alpha} \equiv V_{\alpha}$ для всех $\alpha$. Таким образом,

$$
\mathrm{PA}+\mathrm{Con}(\mathrm{PA}) \equiv_{0} T_{\varepsilon_{0}} \equiv\left(\mathrm{EA}_{\varepsilon_{0}+1}^{+}\right)_{\varepsilon_{0}} .
$$

Свойства функции + для стандартной системы обозначений позволяют установить в ЕА очевидное свойство $\left(U_{\alpha}\right)_{\beta} \equiv U_{\alpha+\beta}$ для любых $\alpha, \beta$. Поэтому $\left(\operatorname{EA}_{\varepsilon_{0}+1}^{+}\right)_{\varepsilon_{0}} \equiv$ $\mathrm{EA}_{\varepsilon_{0}+\varepsilon_{0}}^{+}$, что и требовалось доказать.

\section{СПИСОК ЛИТЕРАТУРЫ}

[1] W. Ackermann. Zur Widerspruchsfreiheit der Zahlentheorie // Math. Ann. 1940. V. 117. P. $162-194$

[2] T. Arai. Epsilon substitution method for theories of jump hierarchies // Arch. Math. Logic. 2002. V. 41. № 2. P. 123-153.

[3] T. Arai. Epsilon substitution method for $\operatorname{ID}_{1}\left(\Pi_{1}^{0} \vee \Sigma_{1}^{0}\right) / /$ Ann. Pure Appl. Logic. 2003. V. 121. № 2-3. P. 163-208.

[4] С.Н. Артёмов. Расширения арифметики и модальные логики // Дисс. ... канд. физ.-матем. наук. М.: МИАН, 1979.

[5] С.Н.Артёмов. Приложения модальной логики в теории доказателств // Вопросы кибернетики: Неклассические логики и их применения. М.: Наука, 1982. С. 3-20.

[6] S. N. Artemov, L. D. Beklemishev. On propositional quantifiers in provability logic // Notre Dame J. Formal Logic. 1993. V. 34. P. 401-419.

[7] S. N. Artemov, L. D. Beklemishev. Provability logic // Handbook of Philosophical Logic. / ed. D. Gabbay, F. Guenthner. V. 13. Dordrecht: Kluwer, 2004. P. 229-403.

[8] J. Avigad. An ordinal analysis of admissible set theory using recursion on ordinal notations // J. Math. Logic. 2002. V. 2. №1. P. 91-112.

[9] J. Avigad, R. Sommer. A model-theoretic approach to ordinal analysis // Bull. Symbolic Logic. 1997. V. 3. № 1. P. 17-52.

[10] J. Avigad, R. Sommer. The model-theoretic ordinal analysis of theories of predicative strength // J. Symbolic Logic. 1999. V. 64. № 1. P. 327-349.

[11] Л. Д. Беклемишев. Об ограниченном правиле индукции и итерированных схемах рефлексии над кальмаровской элементарной арифметикой $/ /$ Теоретические и прикладные аспекты математических исследований / Под ред. О. Б. Лупанова. М.: Изд-во Моск. ун-та, 1994. C. $36-39$. 
[12] L. D. Beklemishev. Iterated local reflection versus iterated consistency // Ann. Pure Appl. Logic. 1995. V. 75. № 1-2. P. 25-48.

[13] L. D. Beklemishev. Remarks on Magari algebras of PA and $I \Delta_{0}+$ EXP // Lecture Notes in Pure and Appl. Math. 1996. V. 180. P. 317-325.

[14] L. D. Beklemishev. Induction rules, reflection principles, and provably recursive functions // Ann. Pure Appl. Logic. 1997. V. 85. № 3. P. 193-242.

[15] L. D. Beklemishev. Notes on local reflection principles // Theoria. 1997. V. 63. № 3. P. 139-146.

[16] L. D. Beklemishev. Parameter free induction and reflection // Lecture Notes in Comput. Sci. 1997. V. 1289. P. 103-113.

[17] L. D. Beklemishev. A proof-theoretic analysis of collection // Arch. Math. Logic. 1998. V. 37. № 5-6. P. 275-296.

[18] L. D. Beklemishev. Parameter free induction and provably total computable functions // Theoret. Comput. Sci. 1999. V. 224. № 1-2. P. 13-33.

[19] L. D. Beklemishev. Proof-theoretic analysis by iterated reflection // Arch. Math. Logic. 2003. V. 42. № 6. P. 515-552.

[20] L. D. Beklemishev. The Worm principle // Logic Group Preprint Ser. V. 219. Utrecht: University of Utrecht, 2003; http://preprints.phil.uu.nl/lgps/.

[21] L. D. Beklemishev. Provability algebras and proof-theoretic ordinals. I // Ann. Pure Appl. Logic. 2004. V. 128. № 1-3. P. 103-123.

[22] G. Boolos. Reflection principles and iterated consistency assertions // J. Symbolic Logic. 1979. V. 44. № 1. P. 33-35.

[23] G. Boolos. The Unprovability of Consistency: An Essay in Modal Logic. Cambridge: Cambridge Univ. Press, 1979.

[24] G. Boolos. Omega-consistency and the diamond // Studia Logica. 1980. V. 39. № 2-3. P. 237-243.

[25] G. Boolos. The Logic of Provability. Cambridge: Cambridge Univ. Press, 1993.

[26] W. Buchholz. Explaining Gentzen's consistency proof within infinitary proof theory // Lecture Notes in Comput. Sci. 1997. V. 1289. P. 4-17.

[27] W. Buchholz. Explaining the Gentzen-Takeuti reduction steps: A second-order system // Arch. Math. Logic. 2001. V. 40. №4. P. 255-272.

[28] W. Buchholz, S. Wainer. Provably computable functions and the fast growing hierarchy // Contemp. Math. 1987. V. 65. P. 179-198.

[29] Н. Катленд. Вычислимость. Введение в теорию рекурсивных функций. М.: Мир, 1983.

[30] Ю. Л. Ершов. Теория нумераций. М.: Наука, 1977.

[31] S. Feferman. Arithmetization of metamathematics in a general setting // Fund. Math. 1960. V. 49. P. 35-92.

[32] S. Feferman. Transfinite recursive progressions of axiomatic theories // J. Symbolic Logic. 1962. V. 27. P. 259-316.

[33] S. Feferman. Three conceptual problems that bug me // Lecture text for 7-th Scandinavian Logic Symposium (Uppsala, 1996); ftp://math.stanford.edu/pub/papers/feferman/; http://math.stanford.edu/ feferman.

[34] G. Gentzen. Die Widerspruchsfreiheit der reinen Zahlentheorie // Math. Ann. 1936. V. 112. P. 493-565.

[35] G. Gentzen. Neue Fassung des Widerspruchsfreiheitsbeweises für die reine Zahlentheorie // Forsch. Logik Grundlegung Exakt. Wiss. 1938. V. 4. P. 19-44.

[36] K. Gödel. Über formal unentscheidbare Sätze der Principia Mathematica und verwandter Systeme. I // Monatsh. Math. Phys. 1931. V. 38. P. 173-198.

[37] С. С. Гончаров. Счетные булевы алгебры и разрешимость. Новосибирск: Научная Книга, 1996. (Сибирская школа алгебры и логики.)

[38] С. В. Горячев. Об интерпретируемости некоторых расширений арифметики // Матем. заметки. 1986. Т. 40. № 5. С. 561-571.

[39] P. Hájek, P. Pudlák. Metamathematics of First-Order Arithmetic. Berlin: Springer-Verlag, 1993. 
[40] L. Henkin, J.D. Monk, A. Tarski. Cylindric algebras. Part I, II. Amsterdam: North-Holland, 1985. (Stud. Logic Found. Math. V. 64, 115.)

[41] K. N. Ignatiev. On strong provability predicates and the associated modal logics // J. Symbolic Logic. 1993. V. 58. № 1. P. 249-290.

[42] Г. К. Джапаридзе. Модально-логические средства исследования доказуемости // Дисс. ... канд. филос. наук. М.: МГУ, 1986.

[43] Г. К. Джапаридзе. Полимодальная логика доказуемости // Интенсиональные логики и логическая структура теорий: Материалы IV Советско-финского симпозиума по логике (Телави, 1985) / Под ред. В.А.Смирнова и М.Н. Бежанишвили. Тбилиси: Мецниереба, 1988. C. $16-48$.

[44] G. Japaridze, D. de Jongh. The logic of provability // Stud. Logic Found. Math. 1998. V. 137. P. $475-546$.

[45] R. Kaye, J. Paris, C. Dimitracopoulos. On parameter free induction schemas // J. Symbolic Logic. 1988. V. 53. № 4. P. 1082-1097.

[46] L. A. S. Kirby, J. B. Paris. Accessible independence results for Peano arithmetic // Bull. London Math. Soc. 1982. V. 14. № 4. P. 285-293.

[47] G. Kreisel. On the interpretation of non-finitist proofs. II: Interpretation of number theory. Applications // J. Symbolic Logic. 1952. V. 17. P. 43-58.

[48] G. Kreisel. A survey of proof theory // J. Symbolic Logic. 1968. V. 33. P. 321-388.

[49] G. Kreisel. Wie die Beweistheorie zu ihren Ordinalzahlen kam und kommt // Jahresber. Deutsch. Math.-Verein. 1976/1977. V. 78. № 4. P. 177-223.

[50] G. Kreisel, A. Lévy. Reflection principles and their use for establishing the complexity of axiomatic systems // Z. Math. Logik Grundlagen Math. 1968. V. 14. P. 97-142.

[51] D. Leivant. The optimality of induction as an axiomatization of arithmetic // J. Symbolic Logic. 1983. V. 48. № 1. P. 182-184.

[52] P. Lindström. On partially conservative sentences and interpretability // Proc. Amer. Math. Soc. 1984. V. 91. № 3. P. 436-443.

[53] P. Lindström. Provability logic-a short introduction // Theoria. 1996. V. 62. № 1-2. P. $19-61$.

[54] M. H. Löb. Solution of a problem of Leon Henkin // J. Symbolic Logic. 1955. V. 20. P. 115-118.

[55] R. Magari. The diagonalizable algebras // Boll. Unione Mat. Ital. (4). 1975. V. 12. № 3. P. $117-125$.

[56] R. Magari. Representation and duality theory for diagonalizable algebras // Studia Logica. 1975. V. 34. № 4. P. 305-313.

[57] Г. Е. Минц. Бескванторные и однокванторные системы // Записки науч. семин. ЛОМИ. 1971. T. 20. C. $115-133$.

[58] G. Mints, S. Tupailo. Epsilon-substitution method for the ramified language and $\Delta_{1}^{1}$-comprehension rule // Synthese Lib. 1999. V. 280. P. 107-130.

[59] F. Montagna. On the algebraization of a Feferman's predicate // Studia Logica. 1978. V. 37. №3. P. 221-236.

[60] F. Montagna. On the diagonalizable algebra of Peano arithmetic // Boll. Unione Mat. Ital. B (5). 1979. V. 16. № 3. P. 795-812.

[61] F. Montagna. The undecidability of the first-order theory of diagonalizable algebras in Peano arithmetic // Studia Logica. 1980. V. 39. № 4. P. 347-354.

[62] H. Ono. Reflection principles in fragments of Peano arithmetic // Z. Math. Logik Grundlagen Math. 1987. V. 33. № 4. P. 317-333.

[63] В. П. Оревков. Нижние оценки удлиннения вьводов после устранени сечения // Записки науч. семин. ЛОМИ. 1979. Т. 88. С. 137-162.

[64] R. Parikh. Existence and feasibility in arithmetic // J. Symbolic Logic. 1971. V. 36. P. $494-508$.

[65] C. Parsons. On a number-theoretic choice schema and its relation to induction // Intuitionism and Proof Theory (Buffalo, 1968) / ed. A. Kino, J. Myhill, R. E. Vessley. Amsterdam: North-Holland, 1970. P. 459-473. 
[66] C. Parsons. On n-quantifier induction // J. Symbolic Logic. 1972. V. 37. № 3. P. 466-482.

[67] W. Pohlers. A short course in ordinal analysis // Proof Theory. Papers from International Summer School and Conference on Proof Theory (Leeds, 1990) / ed. P. Aczel, H. Simmons, S. S. Wainer. Cambridge: Cambridge Univ. Press, 1992. P. 2-78.

[68] W. Pohlers. Subsystems of set theory and second order number theory // Handbook of Proof Theory / ed. S. R. Buss. Amsterdam: North-Holland, 1998. P. 209-335.

[69] M. B. Pour-El, S. Kripke. Deduction-preserving "recursive isomorphisms" between theories // Fund. Math. 1967. V. 61. P. 141-163.

[70] L. J. Pozsgay. Gödel's second theorem for elementary arithmetic // Z. Math. Logik Grundlagen Math. 1968. V. 14. P. 67-80.

[71] M. O. Rabin. Non-standard models and independence of the induction axiom // Essays on the Foundations of Mathematics. Dedicated to A. Fraenkel on his 70th anniversary. Jerusalem: Magnes Press, Hebrew Univ., 1964. P. 287-299.

[72] M. Rathjen. Recent advances in ordinal analysis: $\Pi_{2}^{1}-\mathrm{CA}$ and related systems // Bull. Symbolic Logic. 1995. V. 1. № 4. P. 468-485.

[73] M. Rathjen. The realm of ordinal analysis // London Math. Soc. Lecture Note Ser. 1999. V. 258. P. 219-279.

[74] H. E. Rose. Subrecursion: Functions and Hierarchies. New York: Clarendon Press, 1984.

[75] J. B. Rosser. Extensions of some theorems of Gödel and Church // J. Symbolic Logic. 1936. V. 1. P. $87-91$

[76] J. B. Rosser. Gödel Theorems for non-constructive logics // J. Symbolic Logic. 1937. V. 2. P. 129-137.

[77] U. R. Schmerl. A fine structure generated by reflection formulas over primitive recursive arithmetic // Stud. Logic Found. Math. 1979. V. 97. P. 335-350.

[78] Г. Швихтенберг. Некоторые приложения устранения сечения // Справочная книга по математической логике. Ч. IV. Теория доказательств и конструктивная математика / Под ред. Дж. Барвайса. М.: Наука, 1983. С. 54-83.

[79] K. Segerberg. An Essay in Classical Modal Logic. V. 1-3. Uppsala: Filosofiska Föreningen och Filosofiska Institutionen vid Uppsala Universitet, 1971.

[80] V. Yu. Shavrukov. Subalgebras of diagonalizable algebras of theories containing arithmetic // Dissertationes Math. (Rozprany Mat.). 1993. V. 323. P. 1-82.

[81] V. Yu. Shavrukov. A note on the diagonalizable algebras of PA and ZF // Ann. Pure Appl. Logic. 1993. V. 61. № 1-2. P. 161-173.

[82] V. Yu. Shavrukov. Undecidability in diagonalizable algebras // J. Symbolic Logic. 1997. V. 62. №1. P. 79-116.

[83] V. Yu. Shavrukov. Isomorphisms of diagonalizable algebras // Theoria. 1997. V. 63. № 3. P. 210-221.

[84] К. Сморинский. Теоремы о неполноте // Справочная книга по математической логике. Ч. IV. Теория доказательств и конструктивная математика / Под ред. Дж. Барвайса. М.: Наука, 1983. C. 9-53.

[85] C. Smoryński. $\omega$-consistency and reflection // Colloque International de Logique (Clermont-Ferrand, 1975). Paris: CNRS, 1977. P. 167-181. (Colloq. Internat. CNRS V. 249.)

[86] C. Smoryński. The finite inseparability of the first-order theory of diagonalizable algebras // Studia Logica. 1982. V. 41. № 4. P. 347-349.

[87] C. Smoryński. Self-Reference and Modal Logic. New York: Springer-Verlag, 1985.

[88] R. M. Solovay. Provability interpretations of modal logic // Israel J. Math. 1976. V. 25. № 3-4. P. 287-304.

[89] R. Sommer. Transfiniteinduction within Peano arithmetic // Ann. Pure Appl. Logic. 1995. V. 76. № 3. P. 231-289.

[90] R. Statman. Bounds for proof-search and speed-up in the predicate calculus // Ann. Math. Logic. 1978. V. 15. № 3. P. 225-287.

[91] S. Tupailo. Epsilon substitution method for $\Delta_{1}^{1}$-CR: A constructive termination proof // Log. J. IGPL. 2003. V. 11. № 3. P. 367-377. 
[92] A. M. Turing. Systems of logic based on ordinals // Proc. London Math. Soc. (2). 1939. V. 45. P. $161-228$.

[93] A. Visser. An overview of interpretability logic // Advances in Modal Logic (Berlin, 1996) / ed. M. Kracht et al. V. 1. Stanford: CSLI Publications, 1998. P. 307-359. (CSLI Lecture Notes. V. 87.)

[94] A. Wilkie, J. Paris. On the scheme of induction for bounded arithmetic formulas // Ann. Pure Appl. Logic. 1987. V. 35. № 3. P. 261-302.

[95] D. Zambella. Shavrukov's theorem on the subalgebras of diagonalizable algebras for theories containing $\mathrm{I} \Delta_{0}+\exp / /$ Notre Dame J. Formal Logic. 1994. V. 35. № 1. P. 147-157.

Математический институт им. В. А. Стеклова РАН,

Поступила в редакцию Университет г. Утрехта, Нидерланды

E-mail: bekl@mi.ras.ru 